\title{
CONVERGENCE OF A MIXED METHOD FOR A SEMI-STATIONARY COMPRESSIBLE STOKES SYSTEM
}

\author{
KENNETH H. KARLSEN AND TRYGVE K. KARPER
}

\begin{abstract}
We propose and analyze a finite element method for a semistationary Stokes system modeling compressible fluid flow subject to a Navierslip boundary condition. The velocity (momentum) equation is approximated by a mixed finite element method using the lowest order Nédélec spaces of the first kind, while the continuity equation is approximated by a piecewise constant upwind discontinuous Galerkin method. Our main result states that the numerical method converges to a weak solution. The convergence proof consists of two main steps: (i) To establish strong spatial compactness of the velocity field, which is intricate since the element spaces are only div or curl conforming. (ii) To prove the strong convergence of the discontinuous Galerkin approximations, which is required in view of a nonlinear pressure function. Some tools involved in the analysis include a higher space-time integrability estimate for the discontinuous Galerkin approximations, an equation for the effective viscous flux, various renormalized formulations of the discontinuous Galerkin method, and weak convergence arguments.
\end{abstract}

\section{INTRODUCTION}

The purpose of this paper is to prove convergence of a finite element method for the semi-stationary barotropic compressible Stokes system

$$
\begin{aligned}
\varrho_{t}+\operatorname{div}(\varrho \boldsymbol{u})=0, & & \text { in }(0, T) \times \Omega, \\
-\mu \Delta \boldsymbol{u}-\lambda D \operatorname{div} \boldsymbol{u}+D p(\varrho)=\boldsymbol{f}, & & \text { in }(0, T) \times \Omega,
\end{aligned}
$$

with initial data

$$
\left.\varrho\right|_{t=0}=\varrho_{0}, \quad \text { on } \Omega \text {. }
$$

Here $\Omega$ is a simply-connected, convex, bounded, open, polygonal domain in $\mathbb{R}^{N}$ $(N=2,3)$ with Lipschitz boundary $\partial \Omega$, and $T>0$ is a fixed final time. The unknowns are the density $\varrho=\varrho(t, \boldsymbol{x}) \geq 0$ and the velocity $\boldsymbol{u}=\boldsymbol{u}(t, \boldsymbol{x}) \in \mathbb{R}^{N}$, with $\boldsymbol{x} \in \Omega$ and $t \in(0, T)$. We denote by div and $D$ the usual spatial divergence and gradient operators and by $\Delta$ the spatial Laplace operator.

Received by the editor April 4, 2009 and, in revised form, April 28, 2010.

2010 Mathematics Subject Classification. Primary 35Q30, 74S05; Secondary 65M12.

Key words and phrases. Semi-stationary Stokes system, compressible fluid flow, Navier-slip boundary condition, mixed finite element method, discontinuous Galerkin method, convergence.

The authors thank the anonymous referees for many valuable comments leading to improvements in the paper. This work was supported by the Research Council of Norway through an Outstanding Young Investigators Award (K. H. Karlsen). This article was written as part of the international research program on Nonlinear Partial Differential Equations at the Centre for Advanced Study at the Norwegian Academy of Science and Letters in Oslo during the academic year 2008-09. 
The pressure function is assumed to be of the form $p(\varrho)=a \varrho^{\gamma}$, with $a>0$ (Boyle's law). Typical values of $\gamma$ range from a maximum of $\frac{5}{3}$ for monoatomic gases, through $\frac{7}{5}$ for diatomic gases including air, to lower values close to 1 for polyatomic gases at high temperatures. Throughout this paper we will always assume $\gamma>1$. The case $\gamma=1$ can also be treated; indeed, it is simpler since the pressure function is linear. Furthermore, the viscosity coefficients $\mu, \lambda$ are assumed to be constant and satisfy $\mu>0, N \lambda+2 \mu \geq 0$.

The study of the system (1.1) (1.2) can be motivated in several ways. First, the system can be used as a model equation for the barotropic compressible NavierStokes equations. This can be a reasonable approximation for strongly viscous fluids for which convection can be neglected. Second, Lions 22 use solutions of (1.1)-(1.2) to construct solutions to the full barotropic compressible system.

Among many others, the semi-stationary system (1.1)-11.3) has been studied by Lions in [22, Section 8.2]. He proved the existence of weak solutions and some higher regularity results. Moreover, he proved that weak solutions are unique in the case of periodic boundary conditions or when the equations are solved on the whole of $\mathbb{R}^{N}$. Uniqueness was not obtained in the case of regular Dirichlet boundary conditions and, moreover, higher regularity results were only shown to hold locally.

In this paper we impose the following boundary conditions, which are relevant in the context of geophysical fluids and shallow water models:

$$
\boldsymbol{u} \cdot \nu=0, \quad \text { on }(0, T) \times \partial \Omega,
$$

and

$$
\begin{array}{rll}
\operatorname{curl} \boldsymbol{u}=0, & \text { on }(0, T) \times \partial \Omega, & \text { if } N=2, \\
\operatorname{curl} \boldsymbol{u} \times \nu=0, & \text { on }(0, T) \times \partial \Omega, & \text { if } N=3,
\end{array}
$$

where $\nu$ denotes the unit outward normal to $\partial \Omega$ and curl denotes the curl operator. In $2 \mathrm{D}$, curl denotes the rotation operator taking vectors into scalars (cf. Section 2 ). The first condition is a natural condition of impermeability type on the normal velocity. The second condition is in the literature often referred to as the Navierslip condition. It can be interpreted as a viscous dissipation term at the boundary (more precisely, "non-dissipation" since this term is equal to zero) [22].

In some geophysical applications, conditions like (1.4)-(1.5) are preferred over the classical Dirichlet condition since the latter necessitates expensive calculations of boundary layers. Of more importance to this paper, the boundary conditions (1.4) - 1.5) will allow us to use the finite element method in a solution space that can be split into two orthogonal parts in terms of a discrete version of the Hodge decomposition, a fact that will play a crucial role in our analysis.

Although many numerical methods have been proposed for the compressible Stokes and Navier-Stokes equations, the convergence properties of these methods are mostly unsettled, especially in several spatial dimensions. Ultimately, it is not clear if these numerical methods converge to a weak solution as the discretization parameters tend to zero. In one dimension, the available results are due to Hoff and his collaborators [30, 31, 32. All these results apply to the compressible NavierStokes equations in Lagrangian coordinates, and moreover, they require the initial density to be of bounded variation. Interesting results regarding the existence and long time behavior of solutions to the one-dimensional compressible Navier-Stokes equations have also been obtained using semi-discrete finite difference schemes in 18, 19, 8, again in Lagrangian coordinates with the initial density of bounded total 
variation. In more than one dimension it is a completely open problem to prove convergence of a numerical method for the compressible Navier-Stokes equations. To gain insight into this problem, it is natural to consider simplified models that contain some (but not all) of the difficulties associated with a convergence analysis for the full Navier-Stokes system. The system we consider here, (1.1)-(1.2), is one such example. We also refer to [15] in which a convergent numerical method is proposed for a stationary compressible Stokes system. The system considered in [15] is similar to (1.1)-1.2) but with linear pressure and no temporal dependence.

Regarding a convergence analysis for the system (1.1)-1.2), the main obstacle is to prove strong convergence of the density approximation. Strong convergence is needed to conclude that the pressure term converges. As the density approximation is only known to be uniformly bounded in $L^{2}((0, T) \times \Omega) \cap L^{\infty}\left(0, T ; L^{\gamma}(\Omega)\right)$, strong convergence does not follow by standard compactness arguments. Instead, one is required to adapt the effective viscous flux argument of Lions 22] to the discrete setting. However, the Lions argument implicitly relies on Hodge decompositions. More specifically, it requires the existence of test functions $\boldsymbol{v}_{h}$ satisfying

$$
\int_{\Omega} D \boldsymbol{u}_{h} D \boldsymbol{v}_{h} d x=\int_{\Omega} \operatorname{div} \boldsymbol{u}_{h}\left[\varrho_{h}-\frac{1}{|\Omega|} \int_{\Omega} \varrho_{h} d x\right] d x .
$$

For continuous approximations, $\boldsymbol{v}_{h}=D \Delta^{-1}\left[\varrho_{h}-\frac{1}{|\Omega|} \int_{\Omega} \varrho_{h} d x\right]$ and (1.6) follows from the identity $\int_{\Omega} D \boldsymbol{u}_{h} D \boldsymbol{v}_{h} d x=\int_{\Omega} \operatorname{curl} \boldsymbol{u}_{h} \operatorname{curl} \boldsymbol{v}_{h}+\operatorname{div} \boldsymbol{u}_{h} \operatorname{div} \boldsymbol{v}_{h} d x$. This analysis is hard to replicate at the discrete level. Even though (1.6) does not have to hold exactly, a reminder term must converge to zero without relying on strong convergence (or extra regularity) of $\varrho_{h}$ or $D \boldsymbol{u}_{h}$. In this paper, we introduce the vorticity $\boldsymbol{w}=\operatorname{curl} \boldsymbol{u}$ as an auxiliary variable and rewrite the Laplace operator according to the identity

$$
-\Delta \boldsymbol{u}=\operatorname{curl} \boldsymbol{w}-D \operatorname{div} \boldsymbol{u} .
$$

The viscous flux argument requires the existence of test functions $\boldsymbol{v}_{h}$ satisfying

$$
\int_{\Omega} \operatorname{curl} \boldsymbol{w}_{h} \boldsymbol{v}_{h}+\operatorname{div} \boldsymbol{u}_{h} \operatorname{div} \boldsymbol{v}_{h} d x=\int_{\Omega} \operatorname{div} \boldsymbol{u}_{h}\left[\varrho_{h}-\frac{1}{|\Omega|} \int_{\Omega} \varrho_{h} d x \mid d x .\right.
$$

Consequently, it suffices to find $\boldsymbol{v}_{h}$ satisfying $\operatorname{div} \boldsymbol{v}_{h}=\varrho_{h}-\frac{1}{|\Omega|} \int_{\Omega} \varrho_{h} d x$ and $\int_{\Omega} \operatorname{curl} \boldsymbol{w}_{h} \boldsymbol{v}_{h} d x=0$. For a continuous approximation this is equivalent to (1.6). At the discrete level, (1.7) is easier since several mixed finite element methods are designed for this situation.

Let us now discuss our choice of numerical method for the semi-stationary Stokes system. For the discretization of (1.1) we utilize a discontinuous Galerkin method based on piecewise constant approximations in space and time. The discontinuous Galerkin method was introduced more than 30 years ago [20, 26] and has since then undergone a blooming development; cf. [9, 10, for a review. In the context of linear transport equations with rough (i.e., non-Lipschitz) coefficients, a discontinuous Galerkin method, with piecewise polynomial approximations of arbitrary degree in the spatial variable and piecewise constant or linear approximations in the temporal variable, has recently been analyzed by Walkington in [29]. The work [29] is further developed in 23] for the variable-density incompressible Navier-Stokes equations.

Let us now turn to the velocity (or momentum) equation (1.2). By introducing the vorticity $\boldsymbol{w}=\operatorname{curl} \boldsymbol{u}$ as an auxiliary unknown, keeping in mind the vector 
identity $-\Delta=$ curl curl $-D$ div, we can recast the momentum equation as

$$
\mu \operatorname{curl} \boldsymbol{w}-(\lambda+\mu) D \operatorname{div} \boldsymbol{u}+D p(\varrho)=\boldsymbol{f},
$$

where we suppress the time variable $t$ (we refer the reader to subsequent sections for more precision). Hence the velocity equation (1.2), together with the boundary conditions (1.4)-1.5), admit a formulation that lends itself naturally to a mixed finite element method [17, 24, 25]. Denote by $\boldsymbol{w}_{h} \in \boldsymbol{W}_{h}$ and $\boldsymbol{u}_{h} \in \boldsymbol{V}_{h}$ the mixed finite element approximations, where the spaces $\boldsymbol{V}_{h} \subset \boldsymbol{W}_{0}^{\text {div,2 }}$ and $\boldsymbol{W}_{h} \subset \boldsymbol{W}_{0}^{\text {curl,2 }}$ are based on the Nédélec elements of the first kind 24] (cf. Section 2 for details).

Denote by $\left(\varrho_{h}, \boldsymbol{w}_{h}, \boldsymbol{u}_{h}\right)=\left(\varrho_{h}, \boldsymbol{w}_{h}, \boldsymbol{u}_{h}\right)(t, \boldsymbol{x})$ the numerical solution. The goal is to prove that $\left\{\left(\varrho_{h}, \boldsymbol{w}_{h}, \boldsymbol{u}_{h}\right)\right\}_{h>0}$ converges to a weak solution, at least along a subsequence. The challenging issue is to show that the density approximation $\varrho_{h}$, which on the outset is only weakly compact in $L^{2}$, in fact, converges strongly. Strong convergence is mandatory if we want to recover the semi-stationary Stokes system in the limit when the discretization parameters tend to zero. Related to this issue, the above mixed method enjoys some advantages over the traditional finite element method based on $\boldsymbol{H}^{1}$ elements. In particular, the approximation spaces $\boldsymbol{W}_{h}$ and $\boldsymbol{V}_{h}$ satisfy $(\underline{3})$

$$
\boldsymbol{V}_{h}=\operatorname{curl} \boldsymbol{W}_{h}+\boldsymbol{Z}_{h}
$$

for $\boldsymbol{Z}_{h} \subset \boldsymbol{V}_{h}$ satisfying $\boldsymbol{Z}_{h} \perp$ curl $\boldsymbol{W}_{h}$. An immediate consequence of this discrete Hodge decomposition is that upon writing $\boldsymbol{u}_{h}=\operatorname{curl} \boldsymbol{\eta}_{h}+\boldsymbol{z}_{h}$, we see that only $\boldsymbol{z}_{h}$ is coupled to the density $\varrho_{h}$ and, moreover, that curl $\boldsymbol{w}_{h}$, and hence $\boldsymbol{w}_{h}$, only depends on the data $\boldsymbol{f}$. More importantly, equipped with the discrete Hodge decomposition, we can separate the quantity $P_{\text {eff }}\left(\varrho_{h}, \boldsymbol{u}_{h}\right)=P\left(\varrho_{h}\right)-(\lambda+\mu)$ div $\boldsymbol{u}_{h}$ from the vorticity. The quantity $P_{\text {eff }}\left(\varrho_{h}, \boldsymbol{u}_{h}\right)$ is the so-called effective viscous flux 22] associated with our discrete equations. The fact that we can separate the effective viscous flux from the vorticity makes it possible to prove the following weak continuity property:

$$
\lim _{h \rightarrow 0} \iint P_{\text {eff }}\left(\varrho_{h}, \boldsymbol{u}_{h}\right) \varrho_{h} d x d t=\iint \overline{P_{\text {eff }}} \varrho d x d t \quad\left(\overline{P_{\text {eff }}}, \varrho \text { are weak } L^{2} \text { limits }\right),
$$

which is the decisive ingredient in the proof of strong convergence of the density approximations $\varrho_{h}$. Related to (1.9), we prove a higher integrability estimate on the pressure ensuring that $p\left(\varrho_{h}\right)$, and thus also $P_{\text {eff }}\left(\varrho_{h}, \boldsymbol{u}_{h}\right)$, is weakly compact in $L^{2}$. The energy estimate only provides a uniform bound on $p\left(\varrho_{h}\right)$ in $L^{\infty}\left(L^{1}\right)$, so a priori it is not even clear that $p\left(\varrho_{h}\right)$ converges weakly to an integrable function.

As part of the analysis, we also show that $\varrho_{h} \boldsymbol{u}_{h}$ converges weakly to $\varrho u$, where $\varrho$ and $\boldsymbol{u}$ are weak limits of $\varrho_{h}$ and $\boldsymbol{u}_{h}$, respectively. This convergence is not immediate since the element spaces utilized for the velocity approximations are merely div or curl conforming. In view of the discrete continuity equation (discontinuous Galerkin method), we easily obtain a bound on $\left(\varrho_{h}\right)_{t}$ in, say, $L^{1}\left(W^{-1,1}\right)$. To conclude we need a spatial translation estimate of the form

$$
\left\|\boldsymbol{u}_{h}-\boldsymbol{u}_{h}(\cdot, \cdot+\xi)\right\|_{L^{2}\left(\boldsymbol{L}^{2}\right)} \rightarrow 0 \text { as }|\xi| \rightarrow 0 \text {, uniformly in } h .
$$

In view of the discrete Hodge decomposition, we will actually only need (1.10) for weakly curl free approximations with $\boldsymbol{L}^{2}$ bounded divergence.

For velocity fields that are time-independent, the property (1.10) implies the $\boldsymbol{L}^{2}$ compactness of $\left\{\boldsymbol{u}_{h}\right\}_{h>0}$. In the time independent case, it is known that weakly curl-free approximations with $\boldsymbol{L}^{2}$ bounded divergence are compact in $\boldsymbol{L}^{2}$ provided the approximation spaces satisfy the commuting diagram property [11. Despite the 
fact that the element spaces used here satisfy this property, the inclusion of time in $\boldsymbol{u}_{h}(t, x)$ makes earlier results inadequate. Specifically, to apply known results we would need $L^{\infty}$ control in time of the velocity approximation. Unfortunately, this is not available in general for our problem. As a consequence, we shall provide a direct argument for the spatial translation estimate (1.10).

Finally, we wish to point out that although the boundary conditions (1.4)-(1.5) are not covered by Lions' results 22, his proofs can be adapted to yield existence, uniqueness, and regularity results for (1.1) - 1.2) with the boundary condtions (1.4)(1.5). We will not pursue this project here, except for the existence part, which will be an immediate consequence of our convergence result. However, let us remark that the Navier-slip condition (1.5) is technically easier to handle than a Dirichlet condition, both from a mathematical and numerical point of view. The primary reason for this lies in the need for solutions of the auxiliary problem

$$
\operatorname{div} \boldsymbol{v}=\boldsymbol{f}, \quad \operatorname{curl} \boldsymbol{v}=0 .
$$

If $\int_{\Omega} \boldsymbol{f} d x=0$, the function $\boldsymbol{v}$ will satisfy the boundary conditions (1.4)-(1.5). In other situations, like periodic boundary conditions or when the equations are solved on $\mathbb{R}^{N}$, the boundary values of $\boldsymbol{v}$ do not matter. However, it is evident that $\boldsymbol{v}$ cannot be required to satisfy both Dirichlet boundary conditions and (1.11). Thus, (1.11) can only be required to hold locally whenever Dirichlet boundary conditions are imposed. To avoid "localizing" various discrete arguments, which sometimes can require elaborate work, we have chosen to consider the Navier-slip type condition (1.5) instead of the no-slip Dirichlet condition.

This paper is organized as follows: In Section 2 we introduce notation and list some basic results needed for the analysis. Moreover, we recall the usual notion of weak solution, and also introduce a mixed weak formulation of the velocity equation. Finally, we introduce the finite element spaces and review some of their basic properties. In Section 3, we present the numerical method and state our main convergence result. The existence of a solution to the discrete equations is confirmed in Section 4. Section 5 is devoted to deriving basic a priori estimates. In Section 6. we prove the main convergence result stated in Section 3 The proof is divided into several steps (subsections), including convergence of the continuity scheme, weak continuity of the discrete viscous flux, strong convergence of the density approximations, and convergence of the velocity scheme.

\section{Preliminary material}

2.1. Some functional spaces and analysis results. We will write $W^{m, p}(\Omega)$ to denote the Sobolev space of functions with derivatives of all orders up to $m$ belonging to the space $L^{p}(\Omega)$. To distinguish between scalar and vector functions, we will write vector functions with a bold face. Similarly, a function space written in bold face denotes the vector analog of the corresponding scalar space.

We make frequent use of the divergence and curl operators and denote these by div and curl, respectively. The curl operator is not written in bold face due to its definition in two dimensions. In 2D, we will denote both the rotation operator taking vectors into scalars and the curl operator taking scalars into vectors by curl. Hence, in two dimensions, the definition of curl depends on whether it is applied to a scalar or vector function. Using this slightly confusing notation, we can simultaneously treat the two- and three-dimensional cases. 
We will also make use of the spaces

$$
\begin{aligned}
\boldsymbol{W}^{\operatorname{div}, 2}(\Omega) & =\left\{\boldsymbol{v} \in \boldsymbol{L}^{2}(\Omega): \operatorname{div} \boldsymbol{v} \in L^{2}(\Omega)\right\}, \\
\boldsymbol{W}^{\text {curl, }}(\Omega) & =\left\{\boldsymbol{v} \in \boldsymbol{L}^{2}(\Omega): \operatorname{curl} \boldsymbol{v} \in \boldsymbol{L}^{2}(\Omega)\right\} .
\end{aligned}
$$

If $\boldsymbol{v} \in \boldsymbol{W}^{\text {div,2}}(\Omega)$ satisfies $\left.\boldsymbol{v} \cdot \nu\right|_{\partial \Omega}=0$, where $\nu$ denotes the unit outward pointing normal vector on $\partial \Omega$, we write $\boldsymbol{v} \in \boldsymbol{W}_{0}^{\text {div }, 2}(\Omega)$. Similarly, $\boldsymbol{v} \in \boldsymbol{W}_{0}^{\text {curl,2 }}(\Omega)$ means $\boldsymbol{v} \in \boldsymbol{W}^{\text {curl,2}}(\Omega)$ and $\boldsymbol{v} \times\left.\nu\right|_{\partial \Omega}=0$. In two dimensions, $\boldsymbol{w}$ is a scalar function and the space $\boldsymbol{W}_{0}^{\text {curl, } 2}(\Omega)$ is to be understood as $W_{0}^{1,2}(\Omega)$.

To define weak solutions, we shall use the space

$$
\mathcal{W}(\Omega)=\left\{\boldsymbol{v} \in \boldsymbol{L}^{2}(\Omega): \operatorname{div} \boldsymbol{v} \in L^{2}(\Omega), \operatorname{curl} \boldsymbol{v} \in \boldsymbol{L}^{2}(\Omega),\left.\boldsymbol{v} \cdot \nu\right|_{\partial \Omega}=0\right\},
$$

which coincides with $\boldsymbol{W}_{0}^{\operatorname{div}, 2}(\Omega) \cap \boldsymbol{W}^{\text {curl,2}}(\Omega)$. The space $\mathcal{W}(\Omega)$ is equipped with the $\operatorname{norm}\|\boldsymbol{v}\|_{\mathcal{W}}^{2}=\|\boldsymbol{v}\|_{\boldsymbol{L}^{2}(\Omega)}^{2}+\|\operatorname{div} \boldsymbol{v}\|_{\boldsymbol{L}^{2}(\Omega)}^{2}+\|\operatorname{curl} \boldsymbol{v}\|_{\boldsymbol{L}^{2}(\Omega)}^{2}$. Since $\Omega$ is convex, it is known that $\|\cdot\|_{\mathcal{W}}$ is equivalent to the $\boldsymbol{W}^{1,2}$ norm on the space $\left\{v \in \boldsymbol{W}^{1,2}(\Omega):\left.\boldsymbol{v} \cdot \nu\right|_{\partial \Omega}=0\right\}$; see, e.g., [17.

For the convenience of the reader we list some useful functional analysis results (for proofs, see, e.g.,[14]). Throughout the paper we use overbars to denote weak limits, with the underlying spaces being (silently) given by the context.

Lemma 2.1. Let $O$ be a bounded open subset of $\mathbb{R}^{M}, M \geq 1$. Suppose $g: \mathbb{R} \rightarrow$ $(-\infty, \infty]$ is a lower semi-continuous convex function and $\left\{v_{n}\right\}_{n \geq 1}$ is a sequence of functions on $O$ for which $v_{n} \rightarrow v$ in $L^{1}(O), g\left(v_{n}\right) \in L^{1}(O)$ for each $n, g\left(v_{n}\right) \rightarrow$ $\overline{g(v)}$ in $L^{1}(O)$. Then $g(v) \leq \overline{g(v)}$ a.e. on $O, g(v) \in L^{1}(O)$, and $\int_{O} g(v) d y \leq$ $\liminf _{n \rightarrow \infty} \int_{O} g\left(v_{n}\right) d y$. If, in addition, $g$ is strictly convex on an open interval $(a, b) \subset \mathbb{R}$ and $g(v)=\overline{g(v)}$ a.e. on $O$, then, passing to a subsequence if necessary, $v_{n}(y) \rightarrow v(y)$ for a.e. $y \in\{y \in O \mid v(y) \in(a, b)\}$.

Let $X$ be a Banach space and denote by $X^{\star}$ its dual. The space $X^{\star}$ equipped with the weak- $\star$ topology is denoted by $X_{\text {weak }}^{\star}$, while $X$ equipped with the weak topology is denoted by $X_{\text {weak }}$. By the Banach-Alaoglu theorem, a bounded ball in

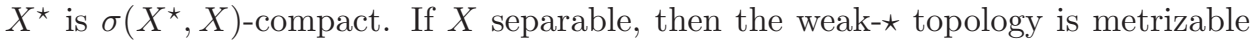
on bounded sets in $X^{\star}$, and thus one can consider the metric space $C\left([0, T] ; X_{\text {weak }}^{\star}\right)$ of functions $v:[0, T] \rightarrow X^{\star}$ that are continuous with respect to the weak topology. We have $v_{n} \rightarrow v$ in $C\left([0, T] ; X_{\text {weak }}^{\star}\right)$ if $\left\langle v_{n}(t), \phi\right\rangle_{X^{\star}, X} \rightarrow\langle v(t), \phi\rangle_{X^{\star}, X}$ uniformly with respect to $t$, for any $\phi \in X$. The following lemma is a consequence of the Arzelà-Ascoli theorem:

Lemma 2.2. Let $X$ be a separable Banach space, and suppose $v_{n}:[0, T] \rightarrow X^{\star}$, $n=1,2, \ldots$, is a sequence for which $\left\|v_{n}\right\|_{L^{\infty}\left([0, T] ; X^{\star}\right)} \leq C$, for some constant $C$ independent of $n$. Suppose the sequence $[0, T] \ni t \mapsto\left\langle v_{n}(t), \Phi\right\rangle_{X^{\star}, X}, n=1,2, \ldots$, is equi-continuous for every $\Phi$ that belongs to a dense subset of $X$. Then $v_{n}$ belongs to $C\left([0, T] ; X_{\text {weak }}^{\star}\right)$ for every $n$, and there exists a function $v \in C\left([0, T] ; X_{\text {weak }}^{\star}\right)$ such that along a subsequence as $n \rightarrow \infty$ there holds $v_{n} \rightarrow v$ in $C\left([0, T] ; X_{\text {weak }}^{\star}\right)$.

In what follows, we will often obtain a priori estimates for a sequence $\left\{v_{n}\right\}_{n \geq 1}$ that we write as " $v_{n} \in_{\mathrm{b}} X$ " for some functional space $X$. What this really means is that we have a bound on $\left\|v_{n}\right\|_{X}$ that is independent of $n$.

The following lemma is Lemma 5.1 in 22 . 
Lemma 2.3. Let $\left\{f_{n}\right\}_{n=1}^{\infty},\left\{g_{n}\right\}_{n=1}^{\infty}$, be sequences of functions on $(0, T) \times \Omega$ such that $f_{n} \rightarrow f$ and $g_{n} \rightarrow g$ in $L^{p_{1}}\left(0, T ; L^{q_{1}}(\Omega)\right)$ and $L^{p_{2}}\left(0, T ; L^{q_{2}}(\Omega)\right)$, respectively, where $\frac{1}{p_{1}}+\frac{1}{p_{2}}=\frac{1}{q_{1}}+\frac{1}{q_{2}}=1$. Assume that $\partial_{t} f_{n} \in{ }_{b} L^{1}\left(0, T ; W^{-m, 1}(\Omega)\right)$, for some $m>0$, and $\left\|g_{n}(t, x)-g_{n}(t, x-\xi)\right\|_{L^{p_{1}}\left(0, T ; L^{q_{2}}(\Omega)\right)} \rightarrow 0$, as $|\xi| \rightarrow 0$, uniformly in $n$. Then $f_{n} g_{n} \rightarrow f g$ in the sense of distributions on $(0, T) \times \Omega$.

2.2. Topological degree in finite dimensions. Our numerical method takes the form of a nonlinear-implicit discrete problem. We will prove the existence of a solution to this problem by a topological degree argument [12.

Denote by $d(F, \Omega, y)$ the $\mathbb{Z}$-valued (Brouwer) degree of a continuous function $F: \bar{O} \rightarrow \mathbb{R}^{M}$ at a point $y \in \mathbb{R}^{M} \backslash F(\partial O)$ relative to an open and bounded set $O \subset \mathbb{R}^{M}$. For notational convenience, let us reformulate the definition of degree so that it applies directly in our finite element setting. Indeed, below we define $d_{S_{h}}\left(F, \tilde{S}_{h}, q_{h}\right)$ with $F: \tilde{S}_{h} \rightarrow S_{h}$ being a continuous finite element mapping, $\tilde{S}_{h}$ being a bounded subset of a finite element space $S_{h}$, and $q_{h}$ being a function in $S_{h}$.

Definition 2.4. Let $S_{h}$ be a finite element space, $\|\cdot\|$ a norm on this space, and introduce the bounded set

$$
\tilde{S}_{h}=\left\{q_{h} \in S_{h} ;\left\|q_{h}\right\| \leq C\right\}
$$

where $C>0$ is a constant. Let $\left\{\sigma_{i}\right\}_{i=1}^{M}$ be a basis such that $\operatorname{span}\left\{\sigma_{i}\right\}_{i=1}^{M}=S_{h}$ and define the operator $\Pi_{\mathcal{B}}: S_{h} \rightarrow \mathbb{R}^{M}$ by

$$
\Pi_{\mathcal{B} q_{h}}=\left(q_{1}, q_{2}, \ldots, q_{M}\right), \quad q_{h}=\sum_{i=1}^{M} q_{i} \sigma_{i} .
$$

The degree $d_{S_{h}}\left(F, \tilde{S}_{h}, q_{h}\right)$ of a continuous mapping $F: \tilde{S}_{h} \rightarrow S_{h}$ at $q_{h} \in S_{h} \backslash F\left(\partial \tilde{S}_{h}\right)$ relative to $\tilde{S}_{h}$ is defined as

$$
d_{S_{h}}\left(F, \tilde{S}_{h}, q_{h}\right)=d\left(\Pi_{\mathcal{B}} F\left(\Pi_{\mathcal{B}}^{-1}\right), \Pi_{\mathcal{B}} \tilde{S}_{h}, \Pi_{\mathcal{B}} q_{h}\right) .
$$

The next lemma is a consequence of some basic properties of the degree; cf. [12].

Lemma 2.5. Fix a finite element space $S_{h}$, and let $d_{S_{h}}\left(F, \tilde{S}_{h}, q_{h}\right)$ be the associated degree of Definition 2.4. The following properties hold:

(1) $d_{S_{h}}\left(F, \tilde{S}_{h}, q_{h}\right)$ does not depend on the choice of basis for $S_{h}$.

(2) $d_{S_{h}}\left(\operatorname{Id}, \tilde{S}_{h}, q_{h}\right)=1$.

(3) $d_{S_{h}}\left(H(\cdot, \alpha), \widetilde{S}_{h}, q_{h}(\alpha)\right)$ is independent of $\alpha \in J:=[0,1]$ for $H: \tilde{S}_{h} \times J \rightarrow S_{h}$ continuous, $q_{h}: J \rightarrow S_{h}$ continuous, and $q_{h}(\alpha) \notin H\left(\partial \widetilde{S}_{h}, \alpha\right) \forall \alpha \in[0,1]$.

(4) $d_{S_{h}}\left(F, \tilde{S}_{h}, q_{h}\right) \neq 0 \Rightarrow F^{-1}\left(q_{h}\right) \neq \emptyset$.

\subsection{Weak and renormalized solutions.}

Definition 2.6 (Weak solutions). We say that a pair of functions $(\varrho, \boldsymbol{u})$ constitutes a weak solution of the semi-stationary compressible Stokes system (1.1)-11.2) with initial data (1.3) and Navier-slip type boundary conditions (1.4)-(1.5) provided the following conditions hold:

(1) $(\varrho, \boldsymbol{u}) \in L^{\infty}\left(0, T ; L^{\gamma}(\Omega)\right) \times L^{2}(0, T ; \mathcal{W}(\Omega))$;

(2) $\varrho_{t}+\operatorname{div}(\varrho \boldsymbol{u})=0$ in the weak sense, i.e, $\forall \phi \in C^{\infty}([0, T) \times \bar{\Omega})$,

$$
\int_{0}^{T} \int_{\Omega} \varrho\left(\phi_{t}+\boldsymbol{u} D \phi\right) d x d t+\left.\int_{\Omega} \varrho_{0} \phi\right|_{t=0} d x=0
$$


(3) $-\mu \Delta \boldsymbol{u}-\lambda D \operatorname{div} \boldsymbol{u}+D p(\varrho)=\boldsymbol{f}$ in the weak sense, i.e, $\forall \boldsymbol{\phi} \in \boldsymbol{C}^{\infty}((0, T) \times \bar{\Omega})$ for which $\phi \cdot \nu=0$ on $(0, T) \times \partial \Omega$,

$\int_{0}^{T} \int_{\Omega} \mu \operatorname{curl} \boldsymbol{u} \operatorname{curl} \boldsymbol{\phi}+[(\mu+\lambda) \operatorname{div} \boldsymbol{u}-p(\varrho)] \operatorname{div} \boldsymbol{\phi} d x d t=\int_{0}^{T} \int_{\Omega} \boldsymbol{f} \boldsymbol{\phi} d x d t$.

For the convergence analysis we shall also need the DiPerna-Lions 13 concept of renormalized solutions of the continuity equation.

Definition 2.7 (Renormalized solutions). Given $\boldsymbol{u} \in L^{2}(0, T ; \mathcal{W}(\Omega))$, we say that $\varrho \in L^{\infty}\left(0, T ; L^{\gamma}(\Omega)\right)$ is a renormalized solution of (1.1) provided

$$
B(\varrho)_{t}+\operatorname{div}(B(\varrho) \boldsymbol{u})+b(\varrho) \operatorname{div} \boldsymbol{u}=0 \quad \text { in the weak sense on }[0, T) \times \bar{\Omega},
$$

for any $B \in C[0, \infty) \cap C^{1}(0, \infty)$ with $B(0)=0$ and $b(\varrho):=\varrho B^{\prime}(\varrho)-B(\varrho)$.

Remark 2.8. Equation (2.2) is formally obtained from the continuity equation (1.1) by multiplying the latter with $B^{\prime}(\varrho)$.

We shall need the following lemma.

Lemma 2.9. Suppose $(\varrho, \boldsymbol{u})$ is a weak solution according to Definition 2.6. If $\left.\varrho \in L^{2}((0, T) \times \Omega)\right)$, then $\varrho$ is a renormalized solution according to Definition 2.7 .

Proof. Let $(\varrho, \boldsymbol{u})$ be a weak solution. Then, since $\Omega$ is convex, $\boldsymbol{u} \in L^{2}\left(0, T ; \boldsymbol{W}^{1,2}(\Omega)\right)$. As the boundary of $\Omega$ is Lipschitz, the velocity field $\boldsymbol{u}(t)$ can be extended to the full space $\mathbb{R}^{N}$ such that $\left.\tilde{\boldsymbol{u}}(t)\right|_{\Omega}=\boldsymbol{u}(t)$ and

$$
\|\tilde{\boldsymbol{u}}\|_{L^{2}\left(0, T ; \boldsymbol{W}^{1,2}\left(\mathbb{R}^{N}\right)\right)} \leq C(\Omega)\|\boldsymbol{u}\|_{L^{2}\left(0, T ; \boldsymbol{W}^{1,2}(\Omega)\right)},
$$

where $\tilde{\boldsymbol{u}}(t)$ denotes the extension of $\boldsymbol{u}(t)$. If we extend $\varrho(t)$ to $\mathbb{R}^{N}$ by setting $\tilde{\varrho}(t)=\varrho(t) \mathbf{1}_{\Omega}$, we get

$$
\tilde{\varrho}_{t}+\operatorname{div}(\tilde{\varrho} \tilde{\boldsymbol{u}})=0 \quad \text { in the weak sense on }[0, T) \times \mathbb{R}^{N} .
$$

Now, to conclude the proof, we appeal to Proposition 4.2 in [14] stating that the square-integrable weak solution $\varrho$ is also a renormalized solution.

2.4. A mixed formulation. In view of the Navier-slip boundary condition (1.5), it is natural to introduce the vorticity $\boldsymbol{w}=\operatorname{curl} \boldsymbol{u}$ as an independent variable, thereby turning the velocity equation into (1.8). This immediately leads to the following mixed formulation, which acts as a motivation for our choice of numerical method: Determine functions

$$
(\boldsymbol{w}, \boldsymbol{u}) \in L^{2}\left(0, T ; \boldsymbol{W}_{0}^{\mathrm{curl}, 2}(\Omega)\right) \times L^{2}\left(0, T ; \boldsymbol{W}_{0}^{\mathrm{div}, 2}(\Omega)\right),
$$

such that

$$
\begin{aligned}
& \int_{0}^{T} \int_{\Omega} \mu \operatorname{curl} \boldsymbol{w} \boldsymbol{v}+[(\mu+\lambda) \operatorname{div} \boldsymbol{u}-p(\varrho)] \operatorname{div} \boldsymbol{v} d x d t=\int_{0}^{T} \int_{\Omega} \boldsymbol{f} \boldsymbol{v} d x d t, \\
& \int_{0}^{T} \int_{\Omega} \boldsymbol{w} \boldsymbol{\eta}-\operatorname{curl} \boldsymbol{\eta} \boldsymbol{u} d x d t=0,
\end{aligned}
$$

for all $(\boldsymbol{\eta}, \boldsymbol{v}) \in L^{2}\left(0, T ; \boldsymbol{W}_{0}^{\text {curl }, 2}(\Omega)\right) \times L^{2}\left(0, T ; \boldsymbol{W}_{0}^{\text {div }, 2}(\Omega)\right)$.

In order to arrive at the weak formulation (2.3), we have utilized the integration by parts formula

$$
\int_{\Omega} \boldsymbol{\eta} \operatorname{curl} \boldsymbol{u} d x=\int_{\Omega} \boldsymbol{u} \operatorname{curl} \boldsymbol{\eta} d x+\int_{\partial \Omega} \boldsymbol{u}(\boldsymbol{\eta} \times \nu) d S(x) .
$$


It follows as an immediate consequence of the Stokes theorem and will be applied multiple times throughout the paper.

The upcoming goal is to prove that a sequence of approximate solutions, denoted by $\left\{\left(\varrho_{h}, \boldsymbol{w}_{h}, \boldsymbol{u}_{h}\right)\right\}_{h>0}$, converges to a limit $(\varrho, \boldsymbol{w}, \boldsymbol{u})$ satisfying (2.1) and (2.3); the term "converges" is made precise in a forthcoming section. Having constructed such a limit, it follows immediately that the pair $(\varrho, \boldsymbol{u})$ is a weak solution according to Definition 2.6. thereby completing the analysis.

2.5. Finite element spaces and some basic results. Throughout this paper, $\left\{E_{h}\right\}_{h}$ denotes a shape regular family of tetrahedral meshes of $\Omega$, where $h$ is the maximal diameter. By shape regular we mean that there exists a constant $\kappa>0$ such that every $E \in E_{h}$ contains a ball of radius $\lambda_{E} \geq \frac{h_{E}}{\kappa}$, where $h_{E}$ is the diameter of $E$. For each fixed $h>0$, we let $\Gamma_{h}$ denote the set of faces in $E_{h}$ and $\mathcal{V}_{h}$ the set of edges. In two dimensions, $\Gamma_{h}$ is the set of edges and $\mathcal{V}_{h}$ the set of vertices. We will use $\mathbb{P}_{j}^{k}(E)$ to denote the space of vector polynomials on $E$ with $k$ components and maximal order $j$.

To approximate the vorticity $\boldsymbol{w}$, we will use the curl-conforming Nédélec space of the first order and kind [24, 7]:

$$
\begin{array}{r}
\boldsymbol{W}_{h}(\Omega)=\left\{\boldsymbol{w} \in \boldsymbol{W}_{0}^{\mathrm{curl}, 2}(\Omega) ;\left.\boldsymbol{w}\right|_{E} \in \boldsymbol{W}(E), \forall E \in E_{h},\right. \\
\left.\int_{e}[\boldsymbol{w} \cdot t] d S(x)=0, \forall e \in \mathcal{V}_{h}\right\},
\end{array}
$$

where $t$ is the unit tangential vector along the edge $e$ and

$$
\boldsymbol{W}(E)= \begin{cases}\mathbb{P}_{1}^{1}(E), & N=2, \\ \left\{\boldsymbol{w} \in \mathbb{P}_{1}^{3}(E) ; D \boldsymbol{w}+D \boldsymbol{w}^{T}=0\right\}, & N=3 .\end{cases}
$$

In two dimensions, the continuity requirement $\int_{e}[\boldsymbol{w} \cdot t] d S(x)=0$ in (2.5) is to be understood as continuity at vertices. For the velocity $\boldsymbol{u}$, we will use the divconforming Nédélec space of the first order and kind [24, 7]:

$$
\boldsymbol{V}_{h}(\Omega)=\left\{\boldsymbol{v} \in \boldsymbol{W}_{0}^{\mathrm{div}, 2} ;\left.\boldsymbol{v}\right|_{E} \in \boldsymbol{V}(E), \forall E \in E_{h}, \int_{\Gamma}[\boldsymbol{v} \cdot \nu] d S(x)=0, \forall \Gamma \in \Gamma_{h}\right\},
$$

where $\boldsymbol{V}(E)=\mathbb{P}_{0}^{N} \oplus \mathbb{P}_{0}^{1} \boldsymbol{x}$. The density $\varrho$ will be approximated in the space of piecewise constants on $E_{h}$ :

$$
Q_{h}(\Omega)=\left\{q \in L^{2}(\Omega) ;\left.q\right|_{E} \in \mathbb{P}_{0}^{1}, \forall E \in E_{h}\right\} .
$$

The spaces $\boldsymbol{W}_{h}(\Omega), \boldsymbol{V}_{h}(\Omega), Q_{h}(\Omega)$ can be related through the following exact sequence (the range of one operator coincides with the kernel of the next) in three dimensions (cf. [2, 3]):

$$
S_{h}(\Omega) \stackrel{\mathrm{grad}}{\longrightarrow} \boldsymbol{W}_{h}(\Omega) \stackrel{\text { curl }}{\longrightarrow} \boldsymbol{V}_{h}(\Omega) \stackrel{\text { div }}{\longrightarrow} Q_{h}(\Omega) / \mathbb{R},
$$

where $S_{h}(\Omega)$ is the linear scalar Lagrange element space. In two dimensions, $S_{h}(\Omega)$ and $\boldsymbol{W}_{h}(\Omega)$ are equal and the sequence does not contain the gradient operator. Since the above sequence is exact, we can define the spaces orthogonal to the range of the previous operator, i.e.,

$$
\begin{aligned}
\boldsymbol{W}_{h}^{0, \perp}(\Omega) & :=\left\{\boldsymbol{w}_{h} \in \boldsymbol{W}_{h}(\Omega) ; \operatorname{curl} \boldsymbol{w}_{h}=0\right\}^{\perp} \cap \boldsymbol{W}_{h}(\Omega)=\left[D S_{h}(\Omega)\right]^{\perp} \cap \boldsymbol{W}_{h}(\Omega), \\
\boldsymbol{V}_{h}^{0, \perp}(\Omega) & :=\left\{\boldsymbol{v}_{h} \in \boldsymbol{V}_{h}(\Omega) ; \operatorname{div} \boldsymbol{v}_{h}=0\right\}^{\perp} \cap \boldsymbol{V}_{h}(\Omega)=\left[\operatorname{curl} \boldsymbol{W}_{h}(\Omega)\right]^{\perp} \cap \boldsymbol{V}_{h}(\Omega),
\end{aligned}
$$


to obtain decompositions

$$
\begin{aligned}
\boldsymbol{W}_{h}(\Omega) & =D S_{h}(\Omega) \oplus \boldsymbol{W}_{h}^{0, \perp}(\Omega), \\
\boldsymbol{V}_{h}(\Omega) & =\operatorname{curl} \boldsymbol{W}_{h}(\Omega) \oplus \boldsymbol{V}_{h}^{0, \perp}(\Omega) .
\end{aligned}
$$

The following discrete Poincaré inequalities hold [2]:

$$
\begin{aligned}
&\left\|\boldsymbol{v}_{h}\right\|_{\boldsymbol{L}^{2}(\Omega)} \leq C\left\|\operatorname{div} \boldsymbol{v}_{h}\right\|_{L^{2}(\Omega)}, \quad \forall \boldsymbol{v} \in \boldsymbol{V}_{h}^{0, \perp}(\Omega), \\
&\left\|\boldsymbol{w}_{h}\right\|_{\boldsymbol{L}^{2}(\Omega)} \leq C\left\|\operatorname{curl} \boldsymbol{w}_{h}\right\|_{L^{2}(\Omega)}, \quad \forall \boldsymbol{w} \in \boldsymbol{W}_{h}^{0, \perp}(\Omega),
\end{aligned}
$$

where the constant $C$ is independent of $h$.

We introduce the canonical interpolation operators:

$$
\begin{aligned}
\Pi_{h}^{W}: \boldsymbol{W}_{0}^{\text {curl,2}}(\Omega) \cap \boldsymbol{W}^{2,2}(\Omega) & \rightarrow \boldsymbol{W}_{h}(\Omega), \quad \Pi_{h}^{V}: \boldsymbol{W}_{0}^{\text {div }, p}(\Omega) \cap \boldsymbol{W}^{1,2}(\Omega) \rightarrow \boldsymbol{V}_{h}(\Omega), \\
\Pi_{h}^{Q}: L^{2}(\Omega) & \rightarrow Q_{h}(\Omega),
\end{aligned}
$$

using the available degrees of freedom of the involved spaces. That is, the operators are defined by [24, 7 ]

$$
\begin{aligned}
\int_{e}\left(\Pi_{h}^{W} \boldsymbol{w}\right) \cdot t d S(x) & =\int_{e} \boldsymbol{w} \cdot t d S(x) \quad \forall e \in \mathcal{V}_{h}, \\
\int_{\Gamma}\left(\Pi_{h}^{V} \boldsymbol{v}\right) \cdot \nu d S(x) & =\int_{\Gamma} \boldsymbol{v} \cdot \nu d S(x) \quad \forall \Gamma \in \Gamma_{h}, \\
\int_{E} \Pi_{h}^{Q} q d x & =\int_{E} q d x, \quad \forall E \in E_{h} .
\end{aligned}
$$

Remark 2.10. The interpolation operators $\Pi_{h}^{W}$ and $\Pi_{h}^{V}$ are defined on function spaces with enough regularity to ensure that the corresponding degrees of freedom are functionals on these spaces (cf. 2, 3]). This is reflected in writing $\boldsymbol{W}_{0}^{\text {curl,2 }} \cap \boldsymbol{W}^{2,2}$ instead of merely $\boldsymbol{W}^{\text {curl,2, }}$, and so on.

The following lemma summarizes well-known error estimates satisfied by the above interpolation operators. The estimates are derived from the Bramble-Hilbert lemma using scaling arguments. However, we note that care must be taken when mapping functions in $\boldsymbol{W}_{h}$ and $\boldsymbol{V}_{h}$ to a reference element (cf. [7, 24]).

Lemma 2.11. There exists a constant $C>0$, depending only on the shape regularity of $E_{h}$ and the size of $\Omega$, such that for any $1 \leq p \leq \infty$,

$$
\begin{aligned}
& \left\|\phi-\Pi_{h}^{Q} \phi\right\|_{L^{p}(\Omega)} \leq C h\|D \phi\|_{\boldsymbol{L}^{p}(\Omega)}, \\
& \left\|\boldsymbol{v}-\Pi_{h}^{V} \boldsymbol{v}\right\|_{\boldsymbol{L}^{p}(\Omega)}+h\left\|\operatorname{div}\left(\boldsymbol{v}-\Pi_{h}^{V} \boldsymbol{v}\right)\right\|_{L^{p}(\Omega)} \leq C h^{s}\left\|D^{s} \boldsymbol{v}\right\|_{\boldsymbol{L}^{p}(\Omega)}, \quad s=1,2, \\
& \left\|\boldsymbol{w}-\Pi_{h}^{W} \boldsymbol{w}\right\|_{\boldsymbol{L}^{p}(\Omega)}+h\left\|\operatorname{curl}\left(\boldsymbol{w}-\Pi_{h}^{W} \boldsymbol{w}\right)\right\|_{\boldsymbol{L}^{p}(\Omega)} \leq C h^{s}\left\|D^{s} \boldsymbol{w}\right\|_{\boldsymbol{L}^{p}(\Omega)}, \quad s=1,2,
\end{aligned}
$$

for all $\phi \in W^{1, p}(\Omega), \boldsymbol{v} \in W^{s, p}(\Omega)$, and $\boldsymbol{w} \in W^{2, p}(\Omega)$.

In what follows, we will need the following lemma. It follows from scaling arguments and the equivalence of finite dimensional norms 4 .

Lemma 2.12. There exists a constant $C>0$ such that for $1 \leq q, p \leq \infty$ and $r=0,1$,

$$
\left\|\phi_{h}\right\|_{W^{r, p}(E)} \leq C h^{-r+N\left(\frac{1}{p}-\frac{1}{q}\right)}\left\|\phi_{h}\right\|_{L^{q}(E)},
$$

for any $E \in E_{h}$ and all polynomial functions $\phi_{h} \in \mathbb{P}_{k}(E), k=0,1, \ldots$. The constant $C$ depends only on the shape regularity of $E_{h}$ and the polynomial degree $k$. 
The next result follows from scaling arguments and the trace theorem [1].

Lemma 2.13. Fix any $E \in E_{h}$ and let $\phi \in W^{1,2}(E)$ be arbitrary. There exists a constant $C>0$, depending only on the shape regularity of $E_{h}$ such that,

$$
\|\phi\|_{L^{2}(\Gamma)} \leq C h^{-\frac{1}{2}}\left(\|\phi\|_{L^{2}(E)}+h\|D \phi\|_{L^{2}(E)}\right), \quad \forall \Gamma \in \Gamma_{h} \cap \partial E .
$$

\section{Numerical method And Main RESult}

In this section we define the numerical method and state the main convergence theorem. The proof of this theorem is deferred to subsequent sections.

Given a time step $\Delta t>0$, we discretize the time interval $[0, T]$ in terms of the points $t^{m}=m \Delta t, m=0, \ldots, M$, where we assume that $M \Delta t=T$. Regarding the spatial discretization, we let $\left\{E_{h}\right\}_{h}$ be a shape regular family of tetrahedral meshes of $\Omega$, where $h$ is the maximal diameter. It will be a standing assumption that $h$ and $\Delta t$ are related such that $\Delta t=c h$, for some constant $c$. Furthermore, we let $\Gamma_{h}$ denote the set of faces in $E_{h}$. Throughout the paper, we will utilize the threedimensional terminology (tetrahedron, face, etc.) for both the three-dimensional case and the two-dimensional case (triangle, edge, etc).

For each fixed $h>0$, we let $\boldsymbol{W}_{h}(\Omega)$ and $\boldsymbol{V}_{h}(\Omega)$ denote the Nédélec spaces of the first order and kind on $E_{h}$ (cf. Section 2.5) and $Q_{h}(\Omega)$ the space of piecewise constants on $E_{h}$. To incorporate boundary conditions, we let the degrees of freedom of $\boldsymbol{W}_{h}(\Omega)$ and $\boldsymbol{V}_{h}(\Omega)$ vanish at the boundary $\partial \Omega$.

Before defining our numerical method, we shall need to introduce some additional notation related to the discontinuous Galerkin method. Concerning the boundary $\partial E$ of an element $E$, we write $f_{+}$for the trace of the function $f$ achieved from within the element $E$ and $f_{-}$for the trace of $f$ achieved from outside $E$. Concerning a face $\Gamma$ that is shared between two elements $E_{-}$and $E_{+}$, we will write $f_{+}$for the trace of $f$ achieved from within $E_{+}$and $f_{-}$for the trace of $f$ achieved from within $E_{-}$. Here $E_{-}$and $E_{+}$are defined such that $\nu$ points from $E_{-}$to $E_{+}$, where $\nu$ is fixed (throughout) as one of the two possible normal components on each face $\Gamma$. We also write $[f]_{\Gamma}=f_{+}-f_{-}$for the jump of $f$ across the face $\Gamma$, while forward time-differencing of $f$ is denoted by $\left[f^{m}\right]=f^{m+1}-f^{m}$. To denote the set of interior faces of $\Gamma_{h}$ we will use the notation $\Gamma_{h}^{I}=\left\{\Gamma \in \Gamma_{h} ; \Gamma \not \subset \partial \Omega\right\}$.

Let us now define our numerical method for the semi-stationary Stokes system (1.1) - (1.2) augmented with the boundary conditions (1.4) and (1.5) (note, however, that in the definition below the boundary conditions are built into the finite element spaces and not stated explicitly).

Definition 3.1 (Numerical method). Let $\left\{\varrho_{h}^{0}(x)\right\}_{h>0}$ be a sequence in $Q_{h}(\Omega)$ that satisfies $\varrho_{h}^{0}>0$ for each fixed $h>0$ and $\varrho_{h}^{0} \rightarrow \varrho_{0}$ a.e. in $\Omega$ and in $L^{1}(\Omega)$ as $h \rightarrow 0$. Set $\boldsymbol{f}_{h}(t, \cdot)=\boldsymbol{f}_{h}^{m}(\cdot):=\frac{1}{\Delta t} \int_{t^{m-1}}^{t^{m}} \Pi_{h}^{Q} \boldsymbol{f}(s, \cdot) d s$, for $t \in\left(t_{m-1}, t_{m}\right), m=1, \ldots, M$.

Now, determine functions

$$
\left(\varrho_{h}^{m}, \boldsymbol{w}_{h}^{m}, \boldsymbol{u}_{h}^{m}\right) \in Q_{h}(\Omega) \times \boldsymbol{W}_{h}(\Omega) \times \boldsymbol{V}_{h}(\Omega), \quad m=1, \ldots, M,
$$

such that for all $\phi_{h} \in Q_{h}(\Omega)$,

$$
\begin{gathered}
\int_{\Omega} \varrho_{h}^{m} \phi_{h} d x-\Delta t \sum_{\Gamma \in \Gamma_{h}^{I}} \int_{\Gamma}\left(\varrho_{-}^{m}\left(\boldsymbol{u}_{h}^{m} \cdot \nu\right)^{+}+\varrho_{+}^{m}\left(\boldsymbol{u}_{h}^{m} \cdot \nu\right)^{-}\right)\left[\phi_{h}\right]_{\Gamma} d S(x) \\
=\int_{\Omega} \varrho_{h}^{m-1} \phi_{h} d x
\end{gathered}
$$


and for all $\left(\boldsymbol{\eta}_{h}, \boldsymbol{v}_{h}\right) \in \boldsymbol{W}_{h}(\Omega) \times \boldsymbol{V}_{h}(\Omega)$,

$$
\begin{aligned}
& \int_{\Omega} \mu \operatorname{curl} \boldsymbol{w}_{h}^{m} \boldsymbol{v}_{h}+\left[(\mu+\lambda) \operatorname{div} \boldsymbol{u}_{h}^{m}-p\left(\varrho_{h}^{m}\right)\right] \operatorname{div} \boldsymbol{v}_{h} d x=\int_{\Omega} \boldsymbol{f}_{h}^{m} \boldsymbol{v}_{h} d x, \\
& \int_{\Omega} \boldsymbol{w}_{h}^{m} \boldsymbol{\eta}_{h}-\boldsymbol{u}_{h}^{m} \operatorname{curl} \boldsymbol{\eta}_{h} d x=0
\end{aligned}
$$

for $m=1, \ldots, M$.

In (3.1), the quantity $\varrho_{-}^{m}\left(\boldsymbol{u}_{h}^{m} \cdot \nu\right)^{+}+\varrho_{+}^{m}\left(\boldsymbol{u}_{h}^{m} \cdot \nu\right)^{-}$is the numerical upwind flux, where $\left(\boldsymbol{u}_{h} \cdot \nu\right)^{+}=\max \left\{\boldsymbol{u}_{h} \cdot \nu, 0\right\}$ and $\left(\boldsymbol{u}_{h} \cdot \nu\right)^{-}=\min \left\{\boldsymbol{u}_{h} \cdot \nu, 0\right\}$, so that there holds $\boldsymbol{u}_{h} \cdot \nu=\left(\boldsymbol{u}_{h} \cdot \nu\right)^{+}+\left(\boldsymbol{u}_{h} \cdot \nu\right)^{-}$. Hence, in the evaluation of $\varrho(\boldsymbol{u} \cdot \nu)$ at the face $\Gamma$ the trace of $\varrho$ is taken in the upwind direction.

Remark 3.2. Recall that $\varrho_{ \pm}$and $\left(\boldsymbol{u}_{h} \cdot \nu\right)^{ \pm}$related to a face $\Gamma$ has a different meaning than $\varrho_{ \pm}$and $\left(\boldsymbol{u}_{h} \cdot \nu\right)^{ \pm}$related to the boundary of an element $\partial E$. Since $\boldsymbol{u}_{h} \cdot \nu$ is constant on each face, one can by direct calculation, verify the identity

$$
\begin{aligned}
& \Delta t \sum_{E \in E_{h}} \int_{\partial E \backslash \partial \Omega}\left(\varrho_{+}^{m}\left(\boldsymbol{u}_{h}^{m} \cdot \nu\right)^{+}+\varrho_{-}^{m}\left(\boldsymbol{u}_{h}^{m} \cdot \nu\right)^{-}\right) \phi_{h} d S(x) \\
& \quad=-\Delta t \sum_{\Gamma \in \Gamma_{h}^{I}} \int_{\Gamma}\left(\varrho_{-}^{m}\left(\boldsymbol{u}_{h}^{m} \cdot \nu\right)^{+}+\varrho_{+}^{m}\left(\boldsymbol{u}_{h}^{m} \cdot \nu\right)^{-}\right)\left[\phi_{h}\right]_{\Gamma} d S(x) .
\end{aligned}
$$

Using this identity, we can state (3.1) in the following form:

$$
\begin{gathered}
\int_{\Omega} \varrho_{h}^{m} \phi_{h} d x+\Delta t \sum_{E \in E_{h}} \int_{\partial E \backslash \partial \Omega}\left(\varrho_{+}^{m}\left(\boldsymbol{u}_{h}^{m} \cdot \nu\right)^{+}+\varrho_{-}^{m}\left(\boldsymbol{u}_{h}^{m} \cdot \nu\right)^{-}\right) \phi_{h} d S(x) \\
=\int_{\Omega} \varrho_{h}^{m-1} \phi_{h} d x .
\end{gathered}
$$

The form (3.3) will be used frequently in the subsequent analysis.

Remark 3.3. For the time discretization of the continuity equation (1.1) we have chosen an implicit Euler method. From an application point of view, however, explicit methods are often preferred over implicit ones when discretizing hyperbolic equations. It is likely that our convergence analysis, with minor modifications, can be applied to an explicit method. To keep the arguments as simple as possible, we will only consider the implicit method (3.1).

Remark 3.4. The discontinuous Galerkin method (3.1) coincides with an upwind finite volume discretization using the degrees of freedom of $\boldsymbol{u}_{h}$ (recall that $\boldsymbol{u}_{h} \cdot \nu$ is constant on each face). This can be seen by setting $\phi_{h}=\chi_{E}, E \in E_{h}$, in (3.3), where $\chi_{E}$ is the characteristic function of $E$. The reader is referred to [16] for a recent paper using this formulation. We refer to 29 for higher order (polynomial) versions of the discontinuous Galerkin method.

For each fixed $h>0$, the numerical solution $\left\{\left(\varrho_{h}^{m}, \boldsymbol{w}_{h}^{m}, \boldsymbol{u}_{h}^{m}\right)\right\}_{m=0}^{M}$ is extended to the whole of $(0, T] \times \Omega$ by setting

$$
\left(\varrho_{h}, \boldsymbol{w}_{h}, \boldsymbol{u}_{h}\right)(t)=\left(\varrho_{h}^{m}, \boldsymbol{w}_{h}^{m}, \boldsymbol{u}_{h}^{m}\right), \quad t \in\left(t_{m-1}, t_{m}\right], \quad m=1, \ldots, M .
$$

In addition, we set $\varrho_{h}(0)=\varrho_{h}^{0}$.

Our main result is that, passing if necessary to a subsequence, $\left\{\left(\varrho_{h}, \boldsymbol{w}_{h}, \boldsymbol{u}_{h}\right)\right\}_{h>0}$ converges to a weak solution. More precisely, there holds 
Theorem 3.5 (Convergence). Suppose $\boldsymbol{f} \in \boldsymbol{L}^{2}((0, T) \times \Omega)$ and $\varrho_{0} \in L^{\gamma}(\Omega), \gamma>1$. Let $\left\{\left(\varrho_{h}, \boldsymbol{w}_{h}, \boldsymbol{u}_{h}\right)\right\}_{h>0}$ be a sequence of numerical solutions constructed according to (3.4) and Definition 3.1. Then, passing if necessary to a subsequence as $h$ tends to zero (assuming $\Delta t=\kappa h, \kappa>0), \boldsymbol{w}_{h} \rightarrow \boldsymbol{w}$ in $L^{2}\left(0, T ; \boldsymbol{W}_{0}^{\text {curl, } 2}(\Omega)\right), \boldsymbol{u}_{h} \rightarrow \boldsymbol{u}$ in $L^{2}\left(0, T ; \boldsymbol{W}_{0}^{\mathrm{div}, 2}(\Omega)\right), \varrho_{h} \boldsymbol{u}_{h} \longrightarrow \varrho \boldsymbol{u}$ in the sense of distributions on $(0, T) \times \Omega$, and $\varrho_{h} \rightarrow \varrho$ a.e. in $(0, T) \times \Omega$, where the limit $(\varrho, \boldsymbol{w}, \boldsymbol{u})$ satisfies the mixed formulation (2.3), and consequently $(\varrho, \boldsymbol{u})$ is a weak solution according to Definition 2.6.

This theorem will be an immediate consequence of the results stated and proved in Sections 46 .

\section{Numerical Method is Well Defined}

In this section we show that there exists a solution to the discrete problem given in Definition 3.1. However, we commence by obtaining a positive lower bound for the density, recalling that the approximate initial density $\varrho_{h}^{0}(\cdot)$ is strictly positive.

Lemma 4.1. Fix any $m=1, \ldots, M$, and suppose $0<\varrho_{h}^{m-1} \in Q_{h}(\Omega), \boldsymbol{u}_{h}^{m} \in \boldsymbol{V}_{h}(\Omega)$ are given bounded functions. Then the solution $\varrho_{h}^{m} \in Q_{h}(\Omega)$ of the discontinuous Galerkin method (3.1) satisfies

$$
\min _{x \in \Omega} \varrho_{h}^{m}(x) \geq \min _{x \in \Omega} \varrho_{h}^{m-1}(x)\left(\frac{1}{1+\Delta t\left\|\operatorname{div} \boldsymbol{u}_{h}^{m}\right\|_{L^{\infty}(\Omega)}}\right)>0 .
$$

Proof. 1. We first prove that $\varrho_{h}^{m}$ is non-negative using an M-matrix argument. For this purpose, let $\mathcal{N}$ denote the number of elements in $E_{h}$ and introduce a numbering of the elements such that $E_{h}=\bigcup_{i=1}^{\mathcal{N}} E_{i}$. Let $\chi_{E_{i}}$ be the characteristic function of $E_{i}$. Then we can express $\varrho_{h}^{m}$ as

$$
\varrho_{h}^{m}=\sum_{i=1}^{\mathcal{N}} \varrho_{i}^{m} \chi_{E_{i}} .
$$

We also define the vector $\varrho^{m}=\left\{\varrho_{i}^{m}\right\}_{i=1}^{\mathcal{N}}$ and note that $\varrho_{i}^{m}=\left.\varrho_{h}^{m}\right|_{E_{i}}$.

Fix $E_{i} \in E_{h}$ and take $\phi_{h}=\chi_{E_{i}}$ in the continuity scheme (3.3), thereby obtaining

$$
\begin{gathered}
\left|E_{i}\right| \varrho_{i}^{m}+\Delta t \int_{\partial E_{i}}\left(\varrho_{+}^{m}\left(\boldsymbol{u}_{h}^{m} \cdot \nu\right)^{+}+\varrho_{-}^{m}\left(\boldsymbol{u}_{h}^{m} \cdot \nu\right)^{-}\right) \chi_{E_{i}} d S(x) \\
=\varrho_{i}^{m}\left[\left|E_{i}\right|+\Delta t \int_{\partial E_{i}}\left(\boldsymbol{u}_{h} \cdot \nu\right)^{+} d S(x)\right]+\Delta t \int_{\partial E_{i}} \varrho_{-}^{m}\left(\boldsymbol{u}_{h}^{m} \cdot \nu\right)^{-} d S(x) \\
=\left[\left|E_{i}\right|+\Delta t \int_{\partial E_{i}}\left(\boldsymbol{u}_{h}^{m} \cdot \nu\right)^{+} d S(x)\right] \varrho_{i}^{m} \\
\quad+\Delta t \sum_{j=1}^{\mathcal{N}}\left(\int_{\partial E_{i} \cap \partial E_{j}}\left(\boldsymbol{u}_{h}^{m} \cdot \nu_{\partial E_{i}}\right)^{-} d S(x)\right) \varrho_{j}^{m},
\end{gathered}
$$

where $\nu_{\partial E_{j}}$ denotes the outward pointing unit normal on $\partial E_{j}$. Since this equation holds for each $i=1, \ldots, \mathcal{N}$, we see that solving the continuity scheme (cf. (3.1) or (3.3) ) is equivalent to solving the linear system

$$
\mathbb{M} \cdot \varrho^{m}=\int_{\Omega} \varrho_{h}^{m-1}\left[\chi_{E_{1}}, \ldots, \chi_{E_{\mathcal{N}}}\right]^{T} d x
$$


where

$$
\begin{aligned}
& \mathbb{M}_{i i}=\left|E_{i}\right|+\Delta t \int_{\partial E_{i}}\left(\boldsymbol{u}_{h}^{m} \cdot \nu\right)^{+} d S(x), \quad i=1, \ldots, \mathcal{N}, \\
& \mathbb{M}_{i j}=\Delta t \int_{\partial E_{i} \cap \partial E_{j}}\left(\boldsymbol{u}_{h}^{m} \cdot \nu_{\partial E_{i}}\right)^{-} d S(x), \quad i, j=1, \ldots, \mathcal{N}, i \neq j .
\end{aligned}
$$

Since $\boldsymbol{u}_{h}^{m} \cdot \nu$ is continuous across faces, we can alternatively write

$$
\mathbb{M}_{i j}=-\Delta t \int_{\partial E_{i} \cap \partial E_{j}}\left(\boldsymbol{u}_{h}^{m} \cdot \nu_{\partial E_{j}}\right)^{+} d S(x), \quad i, j=1, \ldots, \mathcal{N}, i \neq j .
$$

We claim $\mathbb{M}$ is an M-matrix. We recall that a matrix whose off-diagonal entries are real and non-positive is an M-matrix if and only if all its eigenvalues have a non-negative real part (cf. 28]). Clearly, the off-diagonal entries of $\mathbb{M}$ are negative, while the diagonal entries are positive. Moreover, for fixed $j=1, \ldots, \mathcal{N}$, we deduce

$$
\begin{aligned}
\sum_{i=1, i \neq j}^{\mathcal{N}}\left|\mathbb{M}_{i j}\right| & =\Delta t \sum_{i=1, i \neq j}^{\mathcal{N}} \int_{\partial E_{i} \cap \partial E_{j}}\left(\boldsymbol{u}_{h}^{m} \cdot \nu_{\partial E_{j}}\right)^{+} d S(x) \\
& =\Delta t \int_{\partial E_{j}}\left(\boldsymbol{u}_{h}^{m} \cdot \nu\right)^{+} d S(x)<\mathbb{M}_{j j}
\end{aligned}
$$

Hence, the transposed matrix $\mathbb{M}^{T}$ is strictly diagonal dominant, and as a result the real part of the eigenvalues of $\mathbb{M}^{T}$ is strictly positive. Since the eigenvalues of $\mathbb{M}^{T}$ and $\mathbb{M}$ coincide, it follows that $\mathbb{M}$ is an M-matrix. To conclude, now since $\mathbb{M} \cdot \varrho^{m} \geq 0$, there holds $\varrho^{m} \geq 0$; hence $\varrho_{h}^{m}(x) \geq 0 \forall x \in \Omega$.

2. Let $\tilde{E} \in E_{h}$ be such that $\left.\varrho_{h}^{m}\right|_{\tilde{E}} \leq\left.\varrho_{h}^{m}\right|_{E} \forall E \in E_{h}$, and insert into (3.3) the test function $\phi_{h} \in Q_{h}(\Omega)$, defined by

$$
\phi_{h}(x)= \begin{cases}\frac{1}{|\tilde{E}|}, & x \in \tilde{E}, \\ 0, & \text { otherwise }\end{cases}
$$

Integration by parts then yields

$$
\begin{aligned}
\left.\varrho_{h}^{m}\right|_{\tilde{E}} & =-\frac{\Delta t}{|\tilde{E}|} \int_{\partial \tilde{E} \backslash \partial \Omega}\left(\varrho_{+}^{m}\left(\boldsymbol{u}_{h}^{m} \cdot \nu\right)^{+}+\varrho_{-}^{m}\left(\boldsymbol{u}_{h} \cdot \nu\right)^{-}\right) d S(x)+\left.\varrho_{h}^{m-1}\right|_{\tilde{E}} \\
& =-\left.\Delta t\left(\varrho_{h}^{m} \operatorname{div} \boldsymbol{u}_{h}^{m}\right)\right|_{\tilde{E}}-\frac{\Delta t}{|\tilde{E}|} \int_{\partial \tilde{E} \backslash \partial \Omega}\left(\varrho_{-}^{m}-\varrho_{+}^{m}\right)\left(\boldsymbol{u}_{h} \cdot \nu\right)^{-} d S(x)+\left.\varrho_{h}^{m-1}\right|_{\tilde{E}} \\
& \geq-\left.\Delta t\left(\varrho_{h}^{m} \operatorname{div} \boldsymbol{u}_{h}^{m}\right)\right|_{\tilde{E}}+\left.\varrho_{h}^{m-1}\right|_{\tilde{E}},
\end{aligned}
$$

where we have also used the relation $\boldsymbol{u}_{h} \cdot \nu=\left(\boldsymbol{u}_{h} \cdot \nu\right)^{+}+\left(\boldsymbol{u}_{h} \cdot \nu\right)^{-}, \varrho_{h}^{m}$ is constant on $\tilde{E}$, and that $\varrho_{h}^{m}$ attains its minimal value on $\tilde{E}$. Consequently, since $\varrho_{h}^{m}$ is non-negative,

$$
\left.\varrho_{h}^{m}\right|_{\tilde{E}} \geq\left.\varrho_{h}^{m-1}\right|_{\tilde{E}}\left(\frac{1}{1+\Delta t\left\|\operatorname{div} \boldsymbol{u}_{h}^{m}\right\|_{L^{\infty}(\Omega)}}\right)
$$

Remark 4.2. The argument utilized in the second part of the above proof is similar to that found in 29]. Moreover, since the continuity scheme (3.1) is identical to a standard upwind finite volume discretization, the reader can consult [16] for a different approach to obtaining the positivity of the density. 
Remark 4.3. The strict positivity of $\varrho_{h}$ is necessary for the upcoming convergence analysis. More specifically, our arguments will rely on renormalized versions of the continuity scheme (cf. Lemma 5.1), which are not well defined if there are elements $E \in E_{h}$ with $\left.\varrho_{h}\right|_{E}=0$.

We now turn to the question of existence of solutions to our nonlinear-implicit discrete problem. We will apply a topological degree argument, thereby reducing the proof to exhibiting a solution to a linear problem. The proof is similar to an argument employed in [16].

Lemma 4.4. For each fixed $h>0$, there exists a solution

$$
\left(\varrho_{h}^{m}, \boldsymbol{w}_{h}^{m}, \boldsymbol{u}_{h}^{m}\right) \in Q_{h}(\Omega) \times \boldsymbol{W}_{h}(\Omega) \times \boldsymbol{V}_{h}(\Omega), \quad \varrho_{h}^{m}(\cdot)>0, \quad m=1, \ldots, M,
$$

to the nonlinear-implicit discrete problem posed in Definition 3.1 .

Proof. We argue by induction. Assume for $m=1, \ldots, k-1$ that there exists a solution

$$
\left(\varrho_{h}^{m}, \boldsymbol{w}_{h}^{m}, \boldsymbol{u}_{h}^{m}\right) \in S_{h}:=Q_{h}^{+}(\Omega) \times \boldsymbol{W}_{h}(\Omega) \times \boldsymbol{V}_{h}(\Omega)
$$

to the discrete problem of Definition 3.1. Here and below we denote by $Q_{h}^{+}(\Omega)$ the strictly positive functions in $Q_{h}(\Omega)$. Moreover, the norm $\|\cdot\|$ on $S_{h}$ is defined by $\left\|\left(\varrho_{h}, \boldsymbol{w}_{h}, \boldsymbol{u}_{h}\right)\right\|^{2}=\left\|\varrho_{h}\right\|_{L^{2}(\Omega)}^{2}+\left\|\boldsymbol{w}_{h}\right\|_{L^{2}(\Omega)}^{2}+\left\|\boldsymbol{u}_{h}\right\|_{L^{2}(\Omega)}^{2}$.

The claim is that we can find a solution for $m=k$ :

$$
\left(\varrho_{h}^{k}, \boldsymbol{w}_{h}^{k}, \boldsymbol{u}_{h}^{k}\right) \in S_{h} .
$$

To this end, we introduce the mapping

$$
\begin{aligned}
& \boldsymbol{H}: Q_{h}^{+}(\Omega) \times \boldsymbol{W}_{h}(\Omega) \times \boldsymbol{V}_{h}(\Omega) \times[0,1] \rightarrow Q_{h}(\Omega) \times \boldsymbol{W}_{h}(\Omega) \times \boldsymbol{V}_{h}(\Omega), \\
& \boldsymbol{H}\left(\varrho_{h}, \boldsymbol{w}_{h}, \boldsymbol{u}_{h}, \alpha\right)=\left(z_{h}(\alpha), \boldsymbol{y}_{h}(\alpha), \boldsymbol{x}_{h}(\alpha)\right),
\end{aligned}
$$

where the triplet $\left(z_{h}(\alpha), \boldsymbol{y}_{h}(\alpha), \boldsymbol{x}_{h}(\alpha)\right)$ is defined by

$$
\begin{gathered}
\int_{\Omega} z_{h}(\alpha) \phi_{h} d x=\alpha \sum_{E \in E_{h}} \int_{\partial E \backslash \partial \Omega}\left(\varrho_{+}\left(\boldsymbol{u}_{h} \cdot \nu\right)^{+}+\varrho_{-}\left(\boldsymbol{u}_{h} \cdot \nu\right)^{-}\right) \phi_{h} d S(x) \\
\quad+\int_{\Omega} \frac{\varrho_{h}-\varrho_{h}^{k-1}}{\Delta t} \phi_{h} d x, \quad \forall \phi_{h} \in Q_{h}(\Omega), \\
\int_{\Omega} \boldsymbol{x}_{h}(\alpha) \boldsymbol{v}_{h} d x=\int_{\Omega} \mu \operatorname{curl} \boldsymbol{w}_{h} \boldsymbol{v}_{h}+(\lambda+\mu) \operatorname{div} \boldsymbol{u}_{h} \operatorname{div} \boldsymbol{v}_{h} d x \\
-\alpha \int_{\Omega} P\left(\varrho_{h}\right) \operatorname{div} \boldsymbol{v}_{h} d x-\int_{\Omega} \boldsymbol{f}_{h} \boldsymbol{v}_{h} d x, \quad \forall \boldsymbol{v}_{h} \in \boldsymbol{V}_{h}(\Omega), \\
\int_{\Omega} \boldsymbol{y}_{h} \boldsymbol{\eta}_{h} d x=\int_{\Omega} \boldsymbol{w}_{h} \boldsymbol{\eta}_{h}-\boldsymbol{u}_{h} \operatorname{curl} \boldsymbol{\eta}_{h} d x, \quad \forall \boldsymbol{\eta}_{h} \in \boldsymbol{W}_{h}(\Omega) .
\end{gathered}
$$

Solving $\boldsymbol{H}\left(\varrho_{h}, \boldsymbol{u}_{h}, \boldsymbol{w}_{h}, 1\right)=(0,0,0)$ is equivalent to finding a solution (4.4) to the nonlinear-implicit discrete problem posed in Definition 3.1 .

Let us fix an arbitrary $\alpha \in[0,1]$, and consider a solution $\left(\varrho_{h}(\alpha), \boldsymbol{u}_{h}(\alpha), \boldsymbol{w}_{h}(\alpha)\right)$ belonging to $Q_{h}^{+}(\Omega) \times \boldsymbol{W}_{h}(\Omega) \times \boldsymbol{V}_{h}(\Omega)$ of the corresponding problem

$$
\boldsymbol{H}\left(\varrho_{h}(\alpha), \boldsymbol{u}_{h}(\alpha), \boldsymbol{w}_{h}(\alpha), \alpha\right)=(0,0,0) .
$$

1. We claim that there is a constant $C_{\dagger}>0$, independent of $\alpha$, such that

$$
\left\|\boldsymbol{H}\left(\varrho_{h}(\alpha), \boldsymbol{u}_{h}(\alpha), \boldsymbol{w}_{h}(\alpha), \alpha\right)\right\| \leq C_{\dagger} .
$$


To prove (4.8) we utilize (2.7) to write $\boldsymbol{u}_{h}(\alpha)=\operatorname{curl} \boldsymbol{\zeta}_{h}+\boldsymbol{z}_{h}$, for some $\boldsymbol{\zeta}_{\boldsymbol{h}} \in \boldsymbol{W}_{h}^{0, \perp}(\Omega)$ and $\boldsymbol{z}_{h} \in \boldsymbol{V}_{h}^{0, \perp}(\Omega)$. Then, setting $\boldsymbol{\eta}_{h}=\boldsymbol{\zeta}_{h}$ in (4.7) gives

$$
\left\|\operatorname{curl} \boldsymbol{\zeta}_{h}\right\|_{\boldsymbol{L}^{2}(\Omega)}^{2}=\int_{\Omega} \boldsymbol{w}_{h}(\alpha) \boldsymbol{\zeta}_{h} d x \leq \frac{1}{4 \epsilon}\left\|\boldsymbol{w}_{h}(\alpha)\right\|_{\boldsymbol{L}^{2}(\Omega)}^{2}+\epsilon\left\|\boldsymbol{\zeta}_{h}\right\|_{\boldsymbol{L}^{2}(\Omega)}^{2} .
$$

By applying the Poincaré inequality (2.9) to the last term on the right-hand side and fixing $\epsilon$ sufficiently small, we conclude

$$
\left\|\boldsymbol{\zeta}_{h}\right\|_{\boldsymbol{L}^{2}(\Omega)}^{2}+\left\|\operatorname{curl} \boldsymbol{\zeta}_{h}\right\|_{\boldsymbol{L}^{2}(\Omega)}^{2} \leq C\left\|\boldsymbol{w}_{h}(\alpha)\right\|_{\boldsymbol{L}^{2}(\Omega)}^{2} .
$$

From the Poincaré inequality (2.8), we have that $\left\|\boldsymbol{z}_{h}\right\|_{L^{2}(\Omega)} \leq C\left\|\operatorname{div} \boldsymbol{u}_{h}\right\|_{L^{2}(\Omega)}$. Consequently, (4.9) yields

$$
\begin{aligned}
\left\|\boldsymbol{u}_{h}(\alpha)\right\|_{\boldsymbol{L}^{2}(\Omega)}^{2} & =\left\|\operatorname{curl} \boldsymbol{\zeta}_{h}\right\|_{\boldsymbol{L}^{2}(\Omega)}^{2}+\left\|\boldsymbol{z}_{h}\right\|_{\boldsymbol{L}^{2}(\Omega)}^{2} \\
& \leq C\left(\left\|\boldsymbol{w}_{h}(\alpha)\right\|_{\boldsymbol{L}^{2}(\Omega)}^{2}+\left\|\operatorname{div} \boldsymbol{u}_{h}(\alpha)\right\|_{L^{2}(\Omega)}^{2}\right) .
\end{aligned}
$$

Setting $\boldsymbol{v}_{h}=\boldsymbol{u}_{h}(\alpha)$ in (4.6) and adding $\mu$ times (4.7) with $\boldsymbol{\eta}_{h}=\boldsymbol{w}_{h}(\alpha)$, yields

$$
\begin{aligned}
\mu\left\|\boldsymbol{w}_{h}(\alpha)\right\|_{\boldsymbol{L}^{2}(\Omega)}^{2}+(\lambda+\mu)\left\|\operatorname{div} \boldsymbol{u}_{h}(\alpha)\right\|_{L^{2}(\Omega)}^{2} & \int_{\Omega} \alpha P\left(\varrho_{h}(\alpha)\right) \operatorname{div} \boldsymbol{u}_{h}(\alpha)+\boldsymbol{f}_{h} \boldsymbol{u}_{h}(\alpha) d x \\
\leq & \frac{1}{4 \epsilon}\left(\alpha\left\|P\left(\varrho_{h}(\alpha)\right)\right\|_{L^{2}(\Omega)}^{2}+\left\|\boldsymbol{f}_{h}\right\|_{\boldsymbol{L}^{2}(\Omega)}\right) \\
& +\epsilon\left(\left\|\operatorname{div} \boldsymbol{u}_{h}(\alpha)\right\|_{L^{2}(\Omega)}^{2}+\left\|\boldsymbol{u}_{h}(\alpha)\right\|_{L^{2}(\Omega)}^{2}\right) \\
\leq & \frac{1}{4 \epsilon}\left(\alpha\left\|P\left(\varrho_{h}(\alpha)\right)\right\|_{L^{2}(\Omega)}^{2}+\left\|\boldsymbol{f}_{h}\right\|_{L^{2}(\Omega)}\right) \\
& +\epsilon C\left(\left\|\operatorname{div} \boldsymbol{u}_{h}(\alpha)\right\|_{L^{2}(\Omega)}^{2}+\left\|\boldsymbol{w}_{h}(\alpha)\right\|_{\boldsymbol{L}^{2}(\Omega)}^{2}\right),
\end{aligned}
$$

where the last inequality is an application of (4.10). By fixing $\epsilon$ small and applying the Poincaré inequality (2.8), we gather (recall that $\alpha \leq 1$ )

$$
\begin{aligned}
& \left\|\boldsymbol{w}_{h}(\alpha)\right\|_{\boldsymbol{L}^{2}(\Omega)}^{2}+\left\|\boldsymbol{u}_{h}(\alpha)\right\|_{\boldsymbol{L}^{2}(\Omega)}^{2} \\
& \quad \leq C\left(\left\|\varrho_{h}(\alpha)\right\|_{L^{2 \gamma}(\Omega)}^{2 \gamma}+(\Delta t)^{-1}\|\boldsymbol{f}\|_{L^{2}\left(0, T ; \boldsymbol{L}^{2}(\Omega)\right)}^{2}\right) .
\end{aligned}
$$

By the arguments of the previous lemma, we conclude $\varrho_{h}(\alpha)>0$. Hence, setting $\phi_{h} \equiv 1$ in (4.5), yields

$$
\int_{\Omega}\left|\varrho_{h}(\alpha)\right| d x=\int_{\Omega} \varrho_{h}(\alpha) d x=\int_{\Omega} \varrho_{h}^{k-1} d x=\int_{\Omega} \varrho_{h}^{0} d x \leq C .
$$

By the equivalence of finite dimensional norms, we conclude

$$
\left\|\varrho_{h}(\alpha)\right\|_{L^{2}(\Omega)}^{2}+\left\|\varrho_{h}(\alpha)\right\|_{L^{2 \gamma}(\Omega)}^{2 \gamma} \leq C .
$$

Combining (4.11) and (4.12) gives (4.8) and thus we have proved our claim.

2. Let $\widetilde{S}_{h} \subset S_{h}=Q_{h}^{+}(\Omega) \times \boldsymbol{V}_{h}(\Omega) \times \boldsymbol{W}_{h}(\Omega)$ be a ball of sufficiently large radius; cf. (4.8). Then, since every solution of $\boldsymbol{H}\left(\varrho_{h}, \boldsymbol{u}_{h}, \boldsymbol{w}_{h}, \alpha\right)=(0,0,0)$ lies strictly inside $\tilde{S}_{h}$,

$$
(0,0,0) \notin \boldsymbol{H}\left(\partial \tilde{S}_{h}, \alpha\right), \quad \forall \alpha \in[0,1] .
$$

We claim that $\boldsymbol{H}(\cdot, \cdot)$ is continuous on $\tilde{S}_{h} \times[0,1]$. Let $\left(\varrho_{h}, \boldsymbol{u}_{h}, \boldsymbol{w}_{h}\right) \in \tilde{S}_{h}$. By equivalence of norms on finite dimensional spaces, the functions $\varrho_{h}, \boldsymbol{u}_{h}, \boldsymbol{w}_{h}$ are bounded on $\Omega$. In view of this and Lemma 2.12 , the claim follows. 
3. By the virtue of (4.13) and the continuity of $\boldsymbol{H}(\cdot, \cdot)$, we have by Lemma 2.5 that

$$
d_{S_{h}}\left(\boldsymbol{H}(\cdot, \alpha), \tilde{S}_{h}, 0\right) \quad \text { is independent of } \alpha \in[0,1] .
$$

The proof will be completed by proving that $d_{S_{h}}\left(\boldsymbol{H}(\cdot, \alpha=0), \tilde{S}_{h}, 0\right) \neq 0$. To see this, observe that the problem $\boldsymbol{H}\left(\varrho_{h}, \boldsymbol{u}_{h}, \boldsymbol{w}_{h}, 0\right)=(0,0,0)$ is equivalent to finding a triplet $\left(\varrho_{h}, \boldsymbol{u}_{h}, \boldsymbol{w}_{h}\right) \in \tilde{S}_{h}$ satisfying

$$
\int_{\Omega} \varrho_{h} \phi_{h} d x=\int_{\Omega} \varrho_{h}^{k-1} \phi_{h} d x, \quad \forall \phi_{h} \in Q_{h}(\Omega)
$$

and

$$
\begin{gathered}
\int_{\Omega} \mu \operatorname{curl} \boldsymbol{w}_{h} \boldsymbol{v}_{h}+(\lambda+\mu) \operatorname{div} \boldsymbol{u}_{h} \operatorname{div} \boldsymbol{v}_{h} d x=\int_{\Omega} \boldsymbol{f}_{h} \boldsymbol{v}_{h} d x, \quad \forall \boldsymbol{v}_{h} \in \boldsymbol{V}_{h}(\Omega), \\
\int_{\Omega} \operatorname{curl} \boldsymbol{\eta}_{h} \boldsymbol{u}_{h}-\boldsymbol{w}_{h} \boldsymbol{\eta}_{h} d x=0, \quad \forall \boldsymbol{\eta}_{h} \in \boldsymbol{W}_{h}(\Omega) .
\end{gathered}
$$

Clearly, (4.14) has the solution $\varrho_{h}=\varrho_{h}^{k-1}$. Moreover, (4.15) is a system of mixed form admitting a unique solution provided that the finite element spaces satisfy the Babuška-Brezzi condition. It is well known that our choice of finite element spaces satisfies this condition (cf. Theorem A.2).

\section{BASiC ESTIMATES}

In this section we establish a few basic estimates, including square-integrability of the pressure and weak time-continuity of the density. However, we begin by deriving a renormalized formulation of the continuity scheme (3.1).

Lemma 5.1 (Renormalized continuity scheme). Fix any $m=1, \ldots, M$, and let $\left(\varrho_{h}^{m}, \boldsymbol{u}_{h}^{m}\right) \in Q_{h} \times \boldsymbol{V}_{h}$ satisfy the continuity scheme (3.1). Then $\left(\varrho_{h}^{m}, \boldsymbol{u}_{h}^{m}\right)$ also satisfies the renormalized continuity scheme

$$
\begin{aligned}
& \int_{\Omega} B\left(\varrho_{h}^{m}\right) \phi_{h} d x \\
& \quad-\Delta t \sum_{\Gamma \in \Gamma_{h}^{I}} \int_{\Gamma}\left(B\left(\varrho_{-}^{m}\right)\left(\boldsymbol{u}_{h}^{m} \cdot \nu\right)^{+}+B\left(\varrho_{+}^{m}\right)\left(\boldsymbol{u}_{h}^{m} \cdot \nu\right)^{-}\right)\left[\phi_{h}\right]_{\Gamma} d S(x) \\
& \quad+\Delta t \int_{\Omega} b\left(\varrho_{h}^{m}\right) \operatorname{div} \boldsymbol{u}_{h}^{m} \phi_{h} d x+\int_{\Omega} \frac{1}{2} B^{\prime \prime}\left(\xi\left(\varrho_{h}^{m}, \varrho_{h}^{m-1}\right)\right)\left[\varrho_{h}^{m-1}\right]^{2} \phi_{h} d x \\
& \quad+\Delta t \sum_{\Gamma \in \Gamma_{h}^{I}} \int_{\Gamma} \frac{1}{2} B^{\prime \prime}\left(\xi^{\Gamma}\left(\varrho_{+}^{m}, \varrho_{-}^{m}\right)\right)\left[\varrho_{h}^{m}\right]_{\Gamma}^{2}\left(\phi_{h}\right)_{-}\left(\boldsymbol{u}_{h}^{m} \cdot \nu\right)^{+} \\
& \quad-\frac{1}{2} B^{\prime \prime}\left(\xi^{\Gamma}\left(\varrho_{-}^{m}, \varrho_{+}^{m}\right)\right)\left[\varrho_{h}^{m}\right]_{\Gamma}^{2}\left(\phi_{h}\right)_{+}\left(\boldsymbol{u}_{h}^{m} \cdot \nu\right)^{-} d S(x) \\
& =\int_{\Omega} B\left(\varrho_{h}^{m-1}\right) \phi_{h} d x, \quad \forall \phi_{h} \in Q_{h}(\Omega),
\end{aligned}
$$

for any $B \in C[0, \infty) \cap C^{2}(0, \infty)$ and $b(\varrho):=\varrho B^{\prime}(\varrho)-B(\varrho)$. Given two positive real numbers $a_{1}$ and $a_{2}$, we denote by $\xi\left(a_{1}, a_{2}\right)$ and $\xi^{\Gamma}\left(a_{1}, a_{2}\right)$ two numbers between $a_{1}$ and $a_{2}$ (they will be precisely defined below). 
Proof. Since $x \mapsto B^{\prime}\left(\varrho_{h}^{m}(x)\right) \phi_{h}(x)$ is piecewise constant, we can take $B^{\prime}\left(\varrho_{h}^{m}\right) \phi_{h}$ as a test function in the continuity scheme (3.3), yielding

$$
\begin{aligned}
& \int_{\Omega}\left[\varrho_{h}^{m-1}\right] B^{\prime}\left(\varrho_{h}^{m}\right) \phi_{h} d x \\
& =-\Delta t \sum_{E \in E_{h}} \int_{\partial E \backslash \partial \Omega}\left(\varrho_{+}^{m}\left(\boldsymbol{u}_{h}^{m} \cdot \nu\right)^{+}+\varrho_{-}^{m}\left(\boldsymbol{u}_{h}^{m} \cdot \nu\right)^{-}\right) B^{\prime}\left(\varrho_{+}^{m}\right) \phi_{h} d S(x) \\
& =-\Delta t \sum_{E \in E_{h}} \int_{\partial E \backslash \partial \Omega}\left(B^{\prime}\left(\varrho_{+}^{m}\right) \varrho_{+}^{m}\left(\boldsymbol{u}_{h}^{m} \cdot \nu\right)-\left[\varrho_{h}^{m}\right]_{\partial E} B^{\prime}\left(\varrho_{+}^{m}\right)\left(\boldsymbol{u}_{h}^{m} \cdot \nu\right)^{-}\right) \phi_{h} d S(x) .
\end{aligned}
$$

A Taylor expansion yields

$$
B^{\prime}(z)(y-z)=B(y)-B(z)-\frac{1}{2} B^{\prime \prime}\left(z^{*}\right)(y-z)^{2},
$$

for some number $z^{*}$ between $z$ and $y$. Consequently,

$$
\begin{aligned}
& {\left[\varrho_{h}^{m}\right]_{\partial E} B^{\prime}\left(\varrho_{+}^{m}\right)=\left[B\left(\varrho_{h}^{m}\right)\right]_{\partial E}+\frac{1}{2} B^{\prime \prime}\left(\xi^{\partial E}\left(\varrho_{+}^{m}, \varrho_{-}^{m}\right)\right)\left[\varrho_{h}^{m}\right]_{\partial E}^{2},} \\
& {\left[\varrho_{h}^{m-1}\right] B^{\prime}\left(\varrho_{h}^{m}\right)=\left[B\left(\varrho_{h}^{m-1}\right)\right]+\frac{1}{2} B^{\prime \prime}\left(\xi\left(\varrho_{h}^{m}, \varrho_{h}^{m-1}\right)\right)\left[\varrho_{h}^{m-1}\right]^{2},}
\end{aligned}
$$

where

$$
\xi^{\partial E}\left(\varrho_{+}^{m}, \varrho_{-}^{m}\right)(x) \in\left[\varrho_{-}^{m}(x), \varrho_{+}^{m}(x)\right], \quad x \in \partial E
$$

and

$$
\xi\left(\varrho_{h}^{m}, \varrho_{h}^{m-1}\right)(x) \in\left[\varrho_{h}^{m-1}(x), \varrho_{h}^{m}(x)\right], \quad x \in \Omega .
$$

Inserting these identities into (5.2), recalling the definition of $b$, and applying Green's theorem, we achieve

$$
\begin{aligned}
& \int_{\Omega}\left(B\left(\varrho_{h}^{m}\right)-B\left(\varrho_{h}^{m-1}\right)\right) \phi_{h} d x+\int_{\Omega} \frac{1}{2} B^{\prime \prime}\left(\xi\left(\varrho_{h}^{m}, \varrho_{h}^{m-1}\right)\right)\left[\varrho_{h}^{m-1}\right]^{2} \phi_{h} d x \\
& =-\Delta t \int_{\Omega} b\left(\varrho_{h}^{m}\right) \operatorname{div} \boldsymbol{u}_{h}^{m} \phi_{h} d x \\
& \quad-\Delta t \sum_{E \in E_{h}} \int_{\partial E \backslash \partial \Omega}\left(B\left(\varrho_{+}^{m}\right)\left(\boldsymbol{u}_{h}^{m} \cdot \nu\right)^{+}+B\left(\varrho_{-}^{m}\right)\left(\boldsymbol{u}_{h}^{m} \cdot \nu\right)^{-}\right) \phi_{h} d S(x) \\
& \quad+\Delta t \sum_{E \in E_{h}} \int_{\partial E \backslash \partial \Omega} \frac{1}{2} B^{\prime \prime}\left(\xi^{\partial E}\left(\varrho_{+}^{m}, \varrho_{-}^{m}\right)\right)\left[\varrho_{h}^{m}\right]_{\partial E}^{2}\left(\boldsymbol{u}_{h}^{m} \cdot \nu\right)^{-} \phi_{h} d S(x) .
\end{aligned}
$$

Denote by $I$ the second term on the right-hand side of the equality sign. Then, as in Remark 3.2, we have the identity

$$
I=\Delta t \sum_{\Gamma \in \Gamma_{h}^{I}} \int_{\Gamma}\left(B\left(\varrho_{-}^{m}\right)\left(\boldsymbol{u}_{h}^{m} \cdot \nu\right)^{+}+B\left(\varrho_{+}^{m}\right)\left(\boldsymbol{u}_{h}^{m} \cdot \nu\right)^{-}\right)\left[\phi_{h}\right]_{\Gamma} d S(x) .
$$

Recalling that for each $\Gamma \in \Gamma_{h}$ we denote by $E_{+}, E_{-}$the two elements sharing the face $\Gamma$ such that the normal component associated with $\Gamma$ points from $E_{+}$to 
$E_{-}$, we can write

$$
\begin{aligned}
\Delta t \sum_{E \in E_{h}} \int_{\partial E \backslash \partial \Omega} \frac{1}{2} B^{\prime \prime}\left(\xi^{\partial E}\left(\varrho_{+}^{m}, \varrho_{-}^{m}\right)\right)\left[\varrho_{h}^{m}\right]_{\partial E}^{2}\left(\boldsymbol{u}_{h}^{m} \cdot \nu\right)^{-} \phi_{h} d S(x) \\
=\Delta t \sum_{\Gamma \in \Gamma_{h}^{I}} \int_{\Gamma}-\frac{1}{2} B^{\prime \prime}\left(\xi^{\partial E_{-}}\left(\varrho_{+}^{m}, \varrho_{-}^{m}\right)\right)\left[\varrho_{h}^{m}\right]_{\Gamma}^{2}\left(\phi_{h}\right)_{-}\left(\boldsymbol{u}_{h}^{m} \cdot \nu\right)^{+} \\
\quad+\frac{1}{2} B^{\prime \prime}\left(\xi^{\partial E_{+}}\left(\varrho_{+}^{m}, \varrho_{-}^{m}\right)\right)\left[\varrho_{h}^{m}\right]_{\Gamma}^{2}\left(\phi_{h}\right)_{+}\left(\boldsymbol{u}_{h}^{m} \cdot \nu\right)^{-} d S(x) .
\end{aligned}
$$

Once we introduce into (5.5) the notations

$$
\xi^{\Gamma}\left(\varrho_{+}^{m}, \varrho_{-}^{m}\right):=\xi^{\partial E_{+}}\left(\varrho_{+}^{m}, \varrho_{-}^{m}\right), \quad \xi^{\Gamma}\left(\varrho_{-}^{m}, \varrho_{+}^{m}\right):=\xi^{\partial E_{-}}\left(\varrho_{+}^{m}, \varrho_{-}^{m}\right),
$$

inserting (5.4), (5.5) into (5.3) yields the final result (5.1).

In what follows we shall need a discrete Hodge decomposition. The following lemma is a consequence of (2.7).

Lemma 5.2. Let $\left\{\left(\varrho_{h}, \boldsymbol{w}_{h}, \boldsymbol{u}_{h}\right)\right\}_{h>0}$ be a sequence of numerical solutions constructed according to (3.4) and Definition 3.1. For each fixed $h>0$, there exist unique functions $\boldsymbol{\zeta}_{h}^{m} \in \boldsymbol{W}_{h}^{0, \perp}$ and $\boldsymbol{z}_{h}^{m} \in \boldsymbol{V}_{h}^{0, \perp}$ such that

$$
\boldsymbol{u}_{h}^{m}=\operatorname{curl} \boldsymbol{\zeta}_{h}^{m}+\boldsymbol{z}_{h}^{m}, \quad m=1, \ldots, M .
$$

Moreover, if we let $\boldsymbol{\zeta}_{h}(t, x), \boldsymbol{z}_{h}(t, x)$ denote the functions obtained by extending, as in (3.4), $\left\{\boldsymbol{\zeta}_{h}^{m}\right\}_{m=1}^{M},\left\{\boldsymbol{z}_{h}^{m}\right\}_{m=1}^{M}$ to the whole of $(0, T] \times \Omega$, then

$$
\boldsymbol{u}_{h}(t, \cdot)=\operatorname{curl} \boldsymbol{\zeta}_{h}(\cdot, t)+\boldsymbol{z}_{h}(\cdot, t), \quad t \in(0, T) .
$$

We now state a basic stability estimate satisfied by any solution of the discrete problem given in Definition 3.1 .

Lemma 5.3. Let $\left\{\left(\varrho_{h}, \boldsymbol{w}_{h}, \boldsymbol{u}_{h}\right)\right\}_{h>0}$ be a sequence of numerical solutions constructed according to (3.4) and Definition 3.1. For $\varrho>0$, set $P(\varrho):=\frac{a}{\gamma-1} \varrho^{\gamma}$. For any $m=1, \ldots, M$, we have

$$
\begin{aligned}
& \int_{\Omega} P\left(\varrho_{h}^{m}\right) d x \\
& \quad+\sum_{k=1}^{m} \int_{\Omega} \frac{1}{2} P^{\prime \prime}\left(\xi\left(\varrho_{h}^{k}, \varrho_{h}^{k-1}\right)\right)\left[\varrho_{h}^{k-1}\right]^{2} d x \\
& \quad+\sum_{k=1}^{m} \sum_{\Gamma \in \Gamma_{h}^{I}} \Delta t \int_{\Gamma} \frac{1}{2} P^{\prime \prime}\left(\varrho_{\dagger}^{k}\right)\left[\varrho_{h}^{k}\right]_{\Gamma}^{2}\left|\boldsymbol{u}_{h}^{k} \cdot \nu\right| d x \\
& \quad+\sum_{k=1}^{m} \Delta t \int_{\Omega}\left|\boldsymbol{u}_{h}^{k}\right|^{2} d x+\sum_{k=1}^{m} \Delta t \int_{\Omega}\left|\operatorname{div} \boldsymbol{u}_{h}^{k}\right|^{2} d x \\
& \quad+\sum_{k=1}^{m} \Delta t \int_{\Omega}\left|\boldsymbol{w}_{h}^{k}\right|^{2} d x+\sum_{k=1}^{m} \Delta t \int_{\Omega}\left|\operatorname{curl} \boldsymbol{w}_{h}^{k}\right|^{2} d x \\
& \leq \int_{\Omega} P\left(\varrho_{0}\right) d x+C \sum_{k=1}^{m} \Delta t \int_{\Omega}\left|\boldsymbol{f}_{h}^{k}\right|^{2} d x,
\end{aligned}
$$

where $\varrho_{\dagger}^{k}$ is given in (5.9). The constant $C>0$ is independent of $h$ and $\Delta t$, but depends on the coefficients $\mu$ and $\lambda$. 
Consequently, $\varrho_{h} \in{ }_{b} L^{\infty}\left(0, T ; L^{\gamma}(\Omega)\right)$.

Proof. Since $P^{\prime}(\varrho) \varrho-P(\varrho)=p(\varrho)$ and $\varrho_{h}>0$, it follows by taking $\phi_{h} \equiv 1$ in the renormalized scheme (5.1) that

$$
\begin{aligned}
\int_{\Omega} P\left(\varrho_{h}^{k}\right) d x & +\Delta t \int_{\Omega} p\left(\varrho_{h}^{k}\right) \operatorname{div} \boldsymbol{u}_{h}^{k} d x+\int_{\Omega} \frac{1}{2} P^{\prime \prime}\left(\xi\left(\varrho_{h}^{k}, \varrho_{h}^{k-1}\right)\right)\left[\varrho_{h}^{k-1}\right]^{2} d x \\
+ & \Delta t \sum_{\Gamma \in \Gamma_{h}^{I}} \int_{\Gamma} \frac{1}{2} P^{\prime \prime}\left(\xi^{\Gamma}\left(\varrho_{+}^{k}, \varrho_{-}^{k}\right)\right)\left[\varrho_{h}^{k}\right]_{\Gamma}^{2}\left(\boldsymbol{u}_{h}^{k} \cdot \nu\right)^{+} \\
& \quad-\frac{1}{2} P^{\prime \prime}\left(\xi^{\Gamma}\left(\varrho_{-}^{k}, \varrho_{+}^{k}\right)\right)\left[\varrho_{h}^{k}\right]_{\Gamma}^{2}\left(\boldsymbol{u}_{h}^{k} \cdot \nu\right)^{-} d S(x)=\int_{\Omega} P\left(\varrho_{h}^{k-1}\right) d x .
\end{aligned}
$$

For $k=1, \ldots, M$ and $x \in \bigcup_{\Gamma \in \Gamma_{h}^{I}} \Gamma$, set

$$
\varrho_{\dagger}^{k}(x):= \begin{cases}\max \left\{\varrho_{+}^{k}(x), \varrho_{-}^{k}(x)\right\}, & 1<\gamma<2, \\ \min \left\{\varrho_{+}^{k}(x), \varrho_{-}^{k}(x)\right\}, & \gamma \geq 2,\end{cases}
$$

and note that

$$
\begin{gathered}
\Delta t \sum_{\Gamma \in \Gamma_{h}^{I}} \int_{\Gamma} P^{\prime \prime}\left(\xi^{\Gamma}\left(\varrho_{+}^{m}, \varrho_{-}^{m}\right)\right)\left[\varrho_{h}^{k}\right]_{\Gamma}^{2}\left(\boldsymbol{u}_{h}^{k} \cdot \nu\right)^{+} \\
\quad-P^{\prime \prime}\left(\xi^{\Gamma}\left(\varrho_{-}^{m}, \varrho_{+}^{m}\right)\right)\left[\varrho_{h}^{k}\right]_{\Gamma}^{2}\left(\boldsymbol{u}_{h}^{k} \cdot \nu\right)^{-} d S(x), \\
\geq \Delta t \sum_{\Gamma \in \Gamma_{h}^{I}} \int_{\Gamma} P^{\prime \prime}\left(\varrho_{\dagger}^{k}\right)\left[\varrho_{h}^{k}\right]_{\Gamma}^{2}\left|\boldsymbol{u}_{h}^{k} \cdot \nu\right| d S(x) .
\end{gathered}
$$

Next, by using $\boldsymbol{v}_{h}=\boldsymbol{u}_{h}^{k}$ as a test function in the first equation of (3.2) and then using the second equation of (3.2) with $\boldsymbol{\eta}_{h}=\boldsymbol{w}_{h}^{k}$, we obtain the identity

$$
\int_{\Omega} p\left(\varrho_{h}^{k}\right) \operatorname{div} \boldsymbol{u}_{h}^{k} d x=(\mu+\lambda) \int_{\Omega}\left|\operatorname{div} \boldsymbol{u}_{h}^{k}\right|^{2} d x+\mu \int_{\Omega}\left|\boldsymbol{w}_{h}^{k}\right|^{2} d x-\int_{\Omega} \boldsymbol{f}_{h}^{k} \boldsymbol{u}_{h}^{k} d x .
$$

Similarly, specifying $\boldsymbol{v}_{h}=\operatorname{curl} \boldsymbol{w}_{h}^{k}$ in the first equation of (3.2) yields

$$
\mu \int_{\Omega}\left|\operatorname{curl} \boldsymbol{w}_{h}^{k}\right|^{2} d x=\int_{\Omega} \boldsymbol{f}_{h}^{k} \operatorname{curl} \boldsymbol{w}_{h}^{k} d x .
$$

An application of Cauchy's inequality with epsilon $\left(a b \leq \frac{1}{4 \epsilon} a^{2}+\epsilon b^{2}\right)$ then yields

$$
\int_{\Omega}\left|\operatorname{curl} \boldsymbol{w}_{h}^{k}\right|^{2} d x \leq C \int_{\Omega}\left|\boldsymbol{f}_{h}^{k}\right|^{2} d x .
$$

Thanks to (5.6), we can write $\boldsymbol{u}_{h}^{k}=\operatorname{curl} \boldsymbol{\zeta}_{h}^{k}+\boldsymbol{z}_{h}^{k}$ with $\boldsymbol{\zeta}_{h}^{k} \in \boldsymbol{W}_{h}^{0, \perp}$ and $\boldsymbol{z}_{h}^{k} \in \boldsymbol{V}_{h}^{0, \perp}$. Choosing $\boldsymbol{\eta}_{h}=\boldsymbol{\zeta}_{h}^{k}$ in the second equation of (3.2) gives

$$
\int_{\Omega}\left|\operatorname{curl} \boldsymbol{\zeta}_{h}^{k}\right|^{2} d x=\int_{\Omega} \boldsymbol{w}_{h}^{k} \boldsymbol{\zeta}_{h}^{k} d x \leq\left(\int_{\Omega}\left|\boldsymbol{w}_{h}^{k}\right|^{2} d x\right)^{\frac{1}{2}}\left(\int_{\Omega}\left|\boldsymbol{\zeta}_{h}^{k}\right|^{2} d x\right)^{\frac{1}{2}} .
$$

Thus, since the discrete Poincaré inequality (2.9) tells us that

$$
\int_{\Omega}\left|\boldsymbol{\zeta}_{h}^{k}\right|^{2} d x \leq C \int_{\Omega}\left|\operatorname{curl} \boldsymbol{\zeta}_{h}^{k}\right|^{2} d x
$$

we arrive at the estimate

$$
\int_{\Omega}\left|\operatorname{curl} \boldsymbol{\zeta}_{h}^{k}\right|^{2} d x \leq C \int_{\Omega}\left|\boldsymbol{w}_{h}^{k}\right|^{2} d x .
$$


In view of the discrete Poincaré inequality (2.8), we also have

$$
\int_{\Omega}\left|\boldsymbol{z}_{h}^{k}\right|^{2} d x \leq C \int_{\Omega}\left|\operatorname{div} \boldsymbol{u}_{h}^{k}\right|^{2} d x
$$

which, together with (5.13), allow us to conclude

$$
\begin{aligned}
\int_{\Omega}\left|\boldsymbol{u}_{h}^{k}\right|^{2} d x & =\int_{\Omega}\left|\operatorname{curl} \boldsymbol{\zeta}_{h}^{k}\right|^{2}+\left|\boldsymbol{z}_{h}^{k}\right|^{2} d x \\
& \leq C\left(\int_{\Omega}\left|\boldsymbol{w}_{h}^{k}\right|^{2} d x+\int_{\Omega}\left|\operatorname{div} \boldsymbol{u}_{h}^{k}\right|^{2} d x\right) .
\end{aligned}
$$

Now, by first inserting (5.11) into (5.8) and subsequently utilizing (5.10), (5.12), (5.14), and Cauchy's inequality with epsilon to treat the last integral appearing in (5.11), we acquire the estimate

$$
\begin{aligned}
& \int_{\Omega} P\left(\varrho_{h}^{k}\right)-P\left(\varrho_{h}^{k-1}\right) d x \\
& +\int_{\Omega} \frac{1}{2} P^{\prime \prime}\left(\xi\left(\varrho_{h}^{k}, \varrho_{h}^{k-1}\right)\right)\left[\varrho_{h}^{k-1}\right]^{2} d x+\Delta t \sum_{\Gamma \in \Gamma_{h}^{I}} \int_{\Gamma} \frac{1}{2} P^{\prime \prime}\left(\varrho_{\dagger}^{k}\right)\left[\varrho_{h}^{k}\right]_{\Gamma}^{2}\left|\boldsymbol{u}_{h}^{k} \cdot \nu\right| d S(x) \\
& \quad+\Delta t \int_{\Omega}\left|\boldsymbol{u}_{h}^{k}\right|^{2} d x+\Delta t \int_{\Omega}\left|\operatorname{div} \boldsymbol{u}_{h}^{k}\right|^{2} d x \\
& \quad+\Delta t \int_{\Omega}\left|\boldsymbol{w}_{h}^{k}\right|^{2} d x+\Delta t \int_{\Omega}\left|\operatorname{curl} \boldsymbol{w}_{h}^{k}\right|^{2} d x \leq C \Delta t \int_{\Omega}\left|\boldsymbol{f}_{h}^{k}\right|^{2} d x
\end{aligned}
$$

Finally, summing (5.15) over $k$ we conclude that (5.7) holds.

The stability estimate only provides the bound $p\left(\varrho_{h}\right) \in_{b} L^{\infty}\left(0, T ; L^{1}(\Omega)\right)$. Hence, it is not clear that $p\left(\varrho_{h}\right)$ converges weakly to an integrable function. Besides, the subsequent analysis relies heavily on the pressure having higher integrability. In the ensuing lemma we establish that the pressure is in fact bounded in $L^{2}\left(0, T ; L^{2}(\Omega)\right)$.

To simplify the notation, we denote the effective viscous flux by

$$
P_{\text {eff }}\left(\varrho_{h}, \boldsymbol{u}_{h}\right)=p\left(\varrho_{h}\right)-(\mu+\lambda) \operatorname{div} \boldsymbol{u}_{h} .
$$

Lemma 5.4 (Higher integrability on the pressure). Let $\left\{\left(\varrho_{h}, \boldsymbol{w}_{h}, \boldsymbol{u}_{h}\right)\right\}_{h>0}$ be a sequence of numerical solutions constructed according to (3.4) and Definition 3.1. Then

$$
p\left(\varrho_{h}\right) \in_{b} L^{2}((0, T) \times \Omega) .
$$

Proof. For each $m=1, \ldots, M$, let $\boldsymbol{v}_{h}^{m} \in \boldsymbol{V}_{h}^{0, \perp}$ be such that

$$
\operatorname{div} \boldsymbol{v}_{h}^{m}=P_{\text {eff }}\left(\varrho_{h}^{m}, \boldsymbol{u}_{h}^{m}\right)-\frac{1}{|\Omega|} \int_{\Omega} P_{\text {eff }}\left(\varrho_{h}^{m}, \boldsymbol{u}_{h}^{m}\right) d x .
$$

The existence of such functions $\boldsymbol{v}_{h}^{m}$ is immediate from the sequence (2.6).

Now, since the momentum scheme (3.2) gives

$$
\int_{\Omega} P_{\text {eff }}\left(\varrho_{h}^{m}, \boldsymbol{u}_{h}^{m}\right) \operatorname{div} \boldsymbol{v}_{h} d x=-\int_{\Omega} \boldsymbol{f}_{h}^{m} \boldsymbol{v}_{h} d x, \quad \forall \boldsymbol{v}_{h} \in \boldsymbol{V}_{h}^{0, \perp},
$$

we can use $\boldsymbol{v}_{h}^{m}$ as a test function to obtain

$$
\int_{\Omega}\left|P_{\mathrm{eff}}\left(\varrho_{h}^{m}, \boldsymbol{u}_{h}^{m}\right)\right|^{2} d x=\frac{1}{|\Omega|}\left(\int_{\Omega} P_{\mathrm{eff}}\left(\varrho_{h}^{m}, \boldsymbol{u}_{h}^{m}\right) d x\right)^{2}-\int_{\Omega} \boldsymbol{f}_{h}^{m} \boldsymbol{v}_{h}^{m} d x
$$


Hence, with $\epsilon>0$,

$$
\begin{aligned}
& \int_{\Omega}\left|P_{\mathrm{eff}}\left(\varrho_{h}^{m}, \boldsymbol{u}_{h}^{m}\right)-\frac{1}{|\Omega|} \int_{\Omega} P_{\mathrm{eff}}\left(\varrho_{h}^{m}, \boldsymbol{u}_{h}^{m}\right) d x\right|^{2} d x \\
& =-\int_{\Omega} \boldsymbol{f}_{h}^{m} \boldsymbol{v}_{h}^{m} d x \leq \frac{1}{4 \epsilon} \int_{\Omega}\left|\boldsymbol{f}_{h}^{m}\right|^{2} d x+\epsilon \int_{\Omega}\left|\boldsymbol{v}_{h}^{m}\right|^{2} d x .
\end{aligned}
$$

By the Poincaré inequality (2.8),

$$
\int_{\Omega}\left|\boldsymbol{v}_{h}^{m}\right|^{2} d x \leq C \int_{\Omega}\left|P_{\mathrm{eff}}\left(\varrho_{h}^{m}, \boldsymbol{u}_{h}^{m}\right)-\frac{1}{|\Omega|} \int_{\Omega} P_{\mathrm{eff}}\left(\varrho_{h}^{m}, \boldsymbol{u}_{h}^{m}\right) d x\right|^{2} d x .
$$

Consequently, by fixing $\epsilon$ small enough in (5.17),

$$
\int_{\Omega}\left|P_{\mathrm{eff}}\left(\varrho_{h}^{m}, \boldsymbol{u}_{h}^{m}\right)-\frac{1}{|\Omega|} \int_{\Omega} P_{\mathrm{eff}}\left(\varrho_{h}^{m}, \boldsymbol{u}_{h}^{m}\right) d x\right|^{2} d x \leq C \int_{\Omega}\left|\boldsymbol{f}_{h}^{m}\right|^{2} d x,
$$

and thus

$$
\int_{\Omega}\left|P_{\mathrm{eff}}\left(\varrho_{h}^{m}, \boldsymbol{u}_{h}^{m}\right)\right|^{2} d x \leq \frac{1}{|\Omega|}\left(\int_{\Omega} P_{\mathrm{eff}}\left(\varrho_{h}^{m}, \boldsymbol{u}_{h}^{m}\right) d x\right)^{2}+C \int_{\Omega}\left|\boldsymbol{f}_{h}^{m}\right|^{2} d x .
$$

Now, due to the boundary conditions,

$$
\int_{\Omega} P_{\mathrm{eff}}\left(\varrho_{h}^{m}, \boldsymbol{u}_{h}^{m}\right) d x=\int_{\Omega} p\left(\varrho_{h}^{m}\right) d x \leq C,
$$

where we also have put into use Lemma 5.3 and subsequently our assumptions on the source term $f$ and the initial data $\varrho_{0}$. Hence, (5.18) allows us to conclude

$$
\int_{\Omega}\left|P_{\text {eff }}\left(\varrho_{h}^{m}, \boldsymbol{u}_{h}^{m}\right)\right|^{2} d x \leq C\left(1+\int_{\Omega}\left|\boldsymbol{f}_{h}^{m}\right|^{2} d x\right), \quad m=1, \ldots, M,
$$

from which we obtain

$$
\sum_{m=1}^{M} \Delta t \int_{\Omega}\left|P_{\text {eff }}\left(\varrho_{h}^{m}, \boldsymbol{u}_{h}^{m}\right)\right|^{2} d x \leq C\left(T+\sum_{m=1}^{M} \Delta t \int_{\Omega}\left|\boldsymbol{f}_{h}^{m}\right|^{2} d x\right) .
$$

In view of the definition of $P_{\text {eff }}$, this immediately yields

$$
\begin{aligned}
& \sum_{m=1}^{M} \Delta t \int_{\Omega}\left|p\left(\varrho_{h}\right)\right|^{2} d x \\
& \quad \leq C\left(T+\sum_{m=1}^{M} \Delta t \int_{\Omega}\left|\operatorname{div} \boldsymbol{u}_{h}^{m}\right|^{2} d x+\sum_{m=1}^{M} \Delta t \int_{\Omega}\left|\boldsymbol{f}_{h}^{m}\right|^{2} d x\right),
\end{aligned}
$$

which, due to Lemma 5.3 and $\boldsymbol{f} \in L^{2}((0, T) \times \Omega)$, concludes the proof.

We conclude this section by establishing weak time-continuity of the density approximation. For this purpose we shall need the following technical lemma, which provides a bound on the artificial diffusion introduced by the upwind discretization of the continuity equation.

Lemma 5.5. Let $\left\{\left(\varrho_{h}, \boldsymbol{w}_{h}, \boldsymbol{u}_{h}\right)\right\}_{h>0}$ be a sequence of numerical solutions constructed according to (3.4) and Definition 3.1. There exists a constant $C>0$, 
depending only on the shape regularity of $E_{h}$, the size of $\Omega$, the final time $T$, and the data $\varrho_{0}$ and $\boldsymbol{f}$, such that

$$
\begin{aligned}
& \left|\sum_{E \in E_{h}} \int_{0}^{T} \int_{\partial E \backslash \partial \Omega}\left[\varrho_{h}\right]_{\partial E}\left(\boldsymbol{u}_{h} \cdot \nu\right)^{-}\left(\left(\Pi_{h}^{Q} \phi\right)_{+}-\phi\right) d S(x) d t\right| \\
& \quad \leq C\|D \phi\|_{L^{\infty}\left(0, T ; \boldsymbol{L}^{\infty}(\Omega)\right)} h^{\frac{1}{2}}
\end{aligned}
$$

for any $\phi \in L^{\infty}\left(0, T ; W^{1, \infty}(\Omega)\right)$.

Proof. We shall need the auxiliary function

$$
B(z)= \begin{cases}z^{2}, & \gamma>2 \\ z^{\gamma}, & \gamma \leq 2 .\end{cases}
$$

Moreover, set

$$
\phi^{m}(x)=\frac{1}{\Delta t} \int_{t^{m-1}}^{t^{m}} \phi(s, x) d s, \quad \phi_{h}^{m}=\Pi_{h}^{Q} \phi^{m}, \quad m=1, \ldots, M .
$$

Using $\frac{1}{2} B^{\prime \prime}(z)>0$ for $z>0$ and Hölder's inequality, we obtain

$$
\begin{aligned}
I^{2}:= & \left|\sum_{m=1}^{M} \sum_{E \in E_{h}} \Delta t \int_{\partial E \backslash \partial \Omega}\left[\varrho_{h}^{m}\right]_{\partial E}\left(\boldsymbol{u}_{h}^{m} \cdot \nu\right)^{-}\left(\phi_{+}^{m}-\phi^{m}\right) d S(x)\right|^{2} \\
\leq & \left(\sum_{m=1}^{M} \sum_{E \in E_{h}} \Delta t \int_{\partial E \backslash \partial \Omega} \frac{1}{2} B^{\prime \prime}\left(\varrho_{\dagger}^{m}\right)\left[\varrho_{h}^{m}\right]^{2}\left|\boldsymbol{u}_{h}^{m} \cdot \nu\right| d S(x)\right) \\
& \times\left(\sum_{m=1}^{M} \sum_{E \in E_{h}} \Delta t \int_{\partial E \backslash \partial \Omega}\left(\frac{1}{2} B^{\prime \prime}\left(\varrho_{\dagger}^{m}\right)\right)^{-1}\left|\boldsymbol{u}_{h}^{m} \cdot \nu\right|\left|\phi_{+}^{m}-\phi^{m}\right|^{2} d S(x)\right), \\
= & : I_{1} \times I_{2},
\end{aligned}
$$

where the "intermediate" numbers $\varrho_{\dagger}^{m}$ are defined in (5.9).

If $1<\gamma \leq 2$, then Lemma 5.3 can be applied:

$$
I_{1} \leq C \int_{\Omega} B\left(\varrho_{0}\right) d x+\sum_{m=1}^{M} \Delta t \int_{\Omega}\left|\boldsymbol{f}_{h}^{m}\right|^{2} d x
$$

However, (5.21) continues to hold in the case $\gamma \geq 2$. This follows directly from the renormalized scheme (5.1), with $\phi_{h}:=1$, together with the fact that

$$
\begin{aligned}
& \sum_{m=1}^{M} \Delta t \int_{\Omega} b\left(\varrho_{h}^{m}\right) \operatorname{div} \boldsymbol{u}_{h}^{m} d x d t \\
& \quad \leq\left(\sum_{m=1}^{M} \Delta t \int_{\Omega}\left|\varrho_{h}^{m}\right|^{4} d x\right)^{\frac{1}{2}}\left(\sum_{m=1}^{M} \Delta t \int_{\Omega}\left|\operatorname{div} \boldsymbol{u}_{h}^{m}\right|^{2} d x\right)^{\frac{1}{2}}
\end{aligned}
$$

which is bounded by Lemmas 5.3 and 5.4 . 
Next, using Lemma 2.11, we have that

$$
\begin{aligned}
I_{2} \leq h^{2}\|D \phi\|_{L^{\infty}\left(0, T ; \boldsymbol{L}^{\infty}(\Omega)\right)}^{2}\left(\sum_{m=1}^{M}\right. & \left.\sum_{E \in E_{h}} \Delta t \int_{\partial E \backslash \partial \Omega}\left[\left(\frac{1}{2} B^{\prime \prime}\left(\varrho_{\dagger}^{m}\right)\right)^{-1}\right]^{2} d S(x)\right)^{\frac{1}{2}} \\
& \times\left(\sum_{m=1}^{M} \sum_{E \in E_{h}} \Delta t \int_{\partial E \backslash \partial \Omega}\left|\boldsymbol{u}_{h}^{m} \cdot \nu\right|^{2} d S(x)\right)^{\frac{1}{2}}
\end{aligned}
$$

Thanks to Lemma 2.13 and Lemma 2.12.

$$
\int_{\partial E}\left|\boldsymbol{u}_{h}^{m} \cdot \nu\right|^{2} d S(x) \leq c h^{-1} \int_{E}\left|\boldsymbol{u}_{h}^{m}\right|^{2} d x
$$

Moreover, since

$$
\left(\frac{1}{2} B^{\prime \prime}\left(\varrho_{\dagger}^{m}\right)\right)^{-1} \leq C\left|\varrho_{+}^{m}+\varrho_{-}^{m}\right|^{2-\gamma} \leq C\left(1+\varrho_{+}^{m}+\varrho_{-}^{m}\right),
$$

whenever $1<\gamma \leq 2$, and $\left(\frac{1}{2} B^{\prime \prime}\left(\varrho_{\dagger}^{m}\right)\right)^{-1}=\frac{1}{2}$, whenever $\gamma>2$, Lemma 2.13 also gives

$$
\int_{\partial E}\left[\left(\frac{1}{2} B^{\prime \prime}\left(\varrho_{\dagger}^{m}\right)\right)^{-1}\right]^{2} d S(x) \leq C h^{-1}\left(|E|+\int_{E \cup \mathcal{N}(E)}\left|\varrho_{h}^{m}\right|^{2} d x\right),
$$

where $\mathcal{N}(E)$ denotes the union of the neighboring elements of $E$. Observe that

$$
\begin{gathered}
\sum_{E \in E_{h}} h^{-1}\left(|E|+\sum_{F \in \mathcal{N}(E)} \int_{F}\left|\varrho_{h}^{m}\right|^{2} d x\right) \\
\leq h^{-1}\left(|\Omega|+\sum_{E \in E_{h}} \sum_{F \in \mathcal{N}(E) \cup E} \int_{F}\left|\varrho_{h}^{m}\right|^{2} d x\right) \\
\leq h^{-1}\left(|\Omega|+(N+2) \int_{\Omega}\left|\varrho_{h}^{m}\right|^{2} d x\right),
\end{gathered}
$$

where we have utilized the fact that each $E \in E_{h}$ is counted at most $N+2$ times. Inserting (5.23) and (5.24) into (5.22), we have arrived at

$$
\begin{aligned}
& I_{2} \leq h^{2}\|D \phi\|_{L^{\infty}\left(0, T ; \boldsymbol{L}^{\infty}(\Omega)\right)}^{2} h^{-\frac{1}{2}} h^{-\frac{1}{2}} \\
& \times\left(T+\sum_{m=1}^{M} \Delta t \int_{\Omega}\left|\varrho_{h}^{m}\right|^{2} d x\right)^{\frac{1}{2}}\left(\sum_{m=1}^{M} \Delta t \int_{\Omega}\left|\boldsymbol{u}_{h}^{m}\right|^{2} d x\right)^{\frac{1}{2}} \\
& \leq C h\|D \phi\|_{L^{\infty}\left(0, T ; \boldsymbol{L}^{\infty}(\Omega)\right)}^{2},
\end{aligned}
$$

where Lemmas 5.3 and 5.4 have been used to work out the last inequality. This concludes the proof of (5.19).

To simplify the notation, let us introduce the interpolation operator

$$
\left(\Pi_{\mathcal{L}} f\right)(t)=f^{m-1}+\frac{t-t^{m-1}}{\Delta t}\left(f^{m}-f^{m-1}\right), \quad t \in\left(t^{m-1}, t^{m}\right) .
$$


Lemma 5.6. Let $\left\{\left(\varrho_{h}, \boldsymbol{w}_{h}, \boldsymbol{u}_{h}\right)\right\}_{h>0}$ be a sequence of numerical solutions constructed according to (3.4) and Definition 3.1. Then

$$
\frac{\partial}{\partial t}\left(\Pi_{\mathcal{L}} \varrho_{h}\right) \in_{b} L^{1}\left(0, T ; W^{-1,1}(\Omega)\right)
$$

Proof. Fix $\phi \in L^{\infty}\left(0, T ; W^{1, \infty}(\Omega)\right)$ and recall the definitions of $\phi^{m}, \phi_{h}^{m}$; cf. (5.20). The continuity scheme (3.1) with $\phi_{h}^{m}$ as test function reads

$$
\begin{aligned}
& \Delta t \int_{\Omega} \frac{d}{d t}\left(\Pi_{\left.\mathcal{L} \varrho_{h}\right) \phi^{m} d x d t}\right. \\
& \quad=\Delta t \sum_{\Gamma \in \Gamma_{h}^{I}} \int_{\Gamma}\left(\varrho_{-}^{m}\left(\boldsymbol{u}_{h}^{m} \cdot \nu\right)^{+}+\varrho_{+}^{m}\left(\boldsymbol{u}_{h}^{m} \cdot \nu\right)^{-}\right)\left[\phi_{h}^{m}\right]_{\Gamma} d S(x) .
\end{aligned}
$$

Since the traces of $\phi^{m}$ taken from either side of a face are equal, we can write

$$
\begin{aligned}
& \sum_{\Gamma \in \Gamma_{h}^{I}} \int_{\Gamma}\left(\varrho_{-}^{m}\left(\boldsymbol{u}_{h}^{m} \cdot \nu\right)^{+}+\varrho_{+}^{m}\left(\boldsymbol{u}_{h}^{m} \cdot \nu\right)^{-}\right)\left[\phi_{h}^{m}\right]_{\Gamma} d x \\
= & \sum_{\Gamma \in \Gamma_{h}^{I}} \int_{\Gamma}\left(\varrho_{-}^{m}\left(\boldsymbol{u}_{h}^{m} \cdot \nu\right)^{+}+\varrho_{+}^{m}\left(\boldsymbol{u}_{h}^{m} \cdot \nu\right)^{-}\right)\left[\phi_{h}^{m}-\phi^{m}\right]_{\Gamma} d S(x) \\
= & -\sum_{E \in E_{h}} \int_{\partial E \backslash \partial \Omega}\left(\varrho_{+}^{m}\left(\boldsymbol{u}_{h}^{m} \cdot \nu\right)^{+}+\varrho_{-}^{m}\left(\boldsymbol{u}_{h}^{m} \cdot \nu\right)^{-}\right)\left(\phi_{h}^{m}-\phi^{m}\right) d S(x) \\
= & \sum_{E \in E_{h}} \int_{E}-\operatorname{div}\left(\varrho_{h}^{m} \boldsymbol{u}_{h}^{m}\left(\phi_{h}^{m}-\phi^{m}\right)\right) d x \\
& \quad+\sum_{E \in E_{h}} \int_{\partial E \backslash \partial \Omega}\left[\varrho_{h}^{m}\right]_{\partial E}\left(\boldsymbol{u}_{h}^{m} \cdot \nu\right)^{-}\left(\phi_{h}^{m}-\phi^{m}\right) d S(x) \\
= & \sum_{E \in E_{h}} \int_{E}-\varrho_{h}^{m} \operatorname{div} \boldsymbol{u}_{h}^{m}\left(\phi_{h}^{m}-\phi^{m}\right)+\varrho_{h}^{m} \boldsymbol{u}_{h}^{m} \cdot D \phi^{m} d x \\
& \quad+\sum_{E \in E_{h}} \int_{\partial E \backslash \partial \Omega}\left[\varrho_{h}^{m}\right]_{\partial E}\left(\boldsymbol{u}_{h}^{m} \cdot \nu\right)^{-}\left(\phi_{h}^{m}-\phi^{m}\right) d S(x) \\
= & \int_{\Omega} \varrho_{h}^{m} \boldsymbol{u}_{h}^{m} \cdot D \phi^{m} d x+\sum_{E \in E_{h}} \int_{\partial E \backslash \partial \Omega}\left[\varrho_{h}^{m}\right]_{\partial E}\left(\boldsymbol{u}_{h}^{m} \cdot \nu\right)^{-}\left(\phi_{h}^{m}-\phi^{m}\right) d S(x) .
\end{aligned}
$$

To conclude the last equality, we have used

$$
\int_{E} \varrho_{h}^{m} \operatorname{div} \boldsymbol{u}_{h}^{m}\left(\phi_{h}^{m}-\phi^{m}\right) d x=\left.\left(\varrho_{h}^{m} \operatorname{div} \boldsymbol{u}_{h}^{m}\right)\right|_{E} \int_{E} \Pi_{h}^{Q} \phi^{m}-\phi^{m} d x=0, \forall E \in E_{h}
$$

since both $\varrho_{h}^{m}$ and $\operatorname{div} \boldsymbol{u}_{h}^{m}$ are constant on each $E \in E_{h}$. 
By summing (5.26) over $m$, taking absolute values, and using the above identity, we find

$$
\begin{aligned}
\mid \sum_{m=1}^{M} & \Delta t \int_{\Omega} \frac{d}{d t}\left(\Pi_{\mathcal{L}} \varrho_{h}\right) \phi^{m} d x d t \mid \\
\leq & \left|\sum_{m=1}^{M} \Delta t \int_{\Omega} \varrho_{h}^{m} \boldsymbol{u}_{h}^{m} D \phi^{m} d x\right| \\
& +\left|\sum_{m=1}^{M} \sum_{E \in E_{h}} \Delta t \int_{\partial E \backslash \partial \Omega}\left[\varrho_{h}^{m}\right]_{\partial E}\left(\boldsymbol{u}_{h}^{m} \cdot \nu\right)^{-}\left(\phi_{h}^{m}-\phi^{m}\right) d S(x)\right| .
\end{aligned}
$$

Using Lemma 5.5, together with an application of Hölder's inequality, we deduce

$$
\begin{aligned}
& \left|\sum_{m=1}^{M} \Delta t \int_{\Omega} \frac{d}{d t}\left(\Pi_{\mathcal{L}} \varrho_{h}\right) \phi^{m} d x\right| \\
& \leq\left(\sum_{m=1}^{M} \Delta t \int_{\Omega}\left|\varrho_{h}^{m}\right|^{2} d x\right)^{\frac{1}{2}}\left(\sum_{m=1}^{M} \Delta t \int_{\Omega}\left|\boldsymbol{u}_{h}^{m}\right|^{2}\right)^{\frac{1}{2}}\|D \phi\|_{L^{\infty}\left(0, T ; \boldsymbol{L}^{\infty}(\Omega)\right)} \\
& +C h^{\frac{1}{2}}\|D \phi\|_{L^{\infty}\left(0, T ; \boldsymbol{L}^{\infty}(\Omega)\right)} .
\end{aligned}
$$

By Lemmas 5.3 and 5.4 , the first two factors on the right-hand side are bounded, so we conclude that

$$
\begin{aligned}
& \left|\int_{\Delta t}^{T} \int_{\Omega} \frac{d}{d t}\left(\Pi_{\mathcal{L} \varrho_{h}}\right) \phi d x d t\right| \\
& =\left|\sum_{m=1}^{M} \Delta t \int_{\Omega} \frac{d}{d t}\left(\Pi_{\mathcal{L}} \varrho_{h}\right) \phi^{m} d x\right| \leq C\left(1+h^{\frac{1}{2}}\right)\|D \phi\|_{L^{\infty}\left(0, T ; \boldsymbol{L}^{\infty}(\Omega)\right)} .
\end{aligned}
$$

\section{Convergence}

Let $\left\{\left(\varrho_{h}, \boldsymbol{w}_{h}, \boldsymbol{u}_{h}\right)\right\}_{h>0}$ be a sequence of numerical solutions constructed according to (3.4) and Definition 3.1. We will prove that a subsequence of $\left\{\left(\varrho_{h}, \boldsymbol{w}_{h}, \boldsymbol{u}_{h}\right)\right\}_{h>0}$ converges to a weak solution of the semi-stationary Stokes system, thereby proving Theorem 3.5. The proof is divided into several steps:

(1) Convergence of the continuity scheme.

(2) Weak sequential continuity of the discrete viscous flux.

(3) Strong convergence of the density.

(4) Convergence of the velocity scheme.

Our starting point is that the results of Section 5] assure us that the approximate solutions $\left(\boldsymbol{w}_{h}, \boldsymbol{u}_{h}, \varrho_{h}\right)$ satisfy the following $h$-independent bounds:

$$
\varrho_{h} \in_{b} L^{\infty}\left(0, T ; L^{\gamma}(\Omega)\right) \cap L^{2 \gamma}((0, T) \times \Omega)
$$

and

$$
\boldsymbol{w}_{h} \in_{b} L^{2}\left(0, T ; \boldsymbol{W}_{0}^{\mathrm{curl}, 2}(\Omega)\right), \quad \boldsymbol{u}_{h} \in_{b} L^{2}\left(0, T ; \boldsymbol{W}_{0}^{\operatorname{div}, 2}(\Omega)\right) .
$$


Consequently, we may assume that there exist functions $\varrho, \boldsymbol{w}, \boldsymbol{u}$ such that

$$
\begin{aligned}
& \varrho_{h} \stackrel{h \rightarrow 0}{\rightarrow^{0}} \varrho, \quad \text { in } L^{\infty}\left(0, T ; L^{\gamma}(\Omega)\right) \cap L^{2 \gamma}((0, T) \times \Omega), \\
& \boldsymbol{w}_{h} \stackrel{h \rightarrow 0}{ }^{\text {curl, }} \boldsymbol{w}, \quad \text { in } L^{2}\left(0, T ; \boldsymbol{W}_{0}^{\text {curl }}(\Omega),\right. \\
& \boldsymbol{u}_{h}{ }^{h \rightarrow 0} \boldsymbol{u}, \quad \text { in } L^{2}\left(0, T ; \boldsymbol{W}_{0}^{\operatorname{div}, 2}(\Omega)\right) .
\end{aligned}
$$

Moreover,

$$
\varrho_{h}^{\gamma} \stackrel{h \rightarrow 0}{\varrho^{\gamma}}, \quad \varrho_{h}^{\gamma+1} \stackrel{h \rightarrow 0}{\varrho^{\gamma+1}}, \quad \varrho_{h} \log \varrho_{h}{ }^{h \rightarrow 0} \bar{\varrho} \overline{\log \varrho},
$$

where each $\stackrel{h \rightarrow 0}{ }^{0}$ signifies weak convergence in a suitable $L^{p}$ space with $p>1$.

Finally, $\varrho_{h}, \varrho_{h} \log \varrho_{h}$ converge respectively to $\varrho, \overline{\varrho \log \varrho}$ in $C\left([0, T] ; L_{\text {weak }}^{p}(\Omega)\right)$ for some $1<p<\gamma$; cf. Lemma 2.2 and also [14, 22. In particular, $\varrho$, $\varrho \log \varrho$, and $\overline{\varrho \log \varrho}$ belong to $C\left([0, T] ; L_{\text {weak }}^{p}(\Omega)\right)$.

6.1. Density scheme. To prove that the weak limit $(\varrho, u)$ is a weak solution to the continuity equation (1.1), the main difficulty is to obtain convergence of the product $\varrho_{h} \boldsymbol{u}_{h} \rightarrow \varrho \boldsymbol{u}$. In Lemma 6.3 below, this will be achieved by adapting the proof of Lemma 2.3 to our discrete case, where $\varrho_{h}$ and $\boldsymbol{u}_{h}$ play the roles of $f_{n}$ and $g_{n}$, respectively.

From Lemma 5.6. $\frac{\partial}{\partial t}\left(\Pi_{\mathcal{L} \varrho_{h}}\right) \in_{b} L^{1}\left(0, T ; W^{-1,1}(\Omega)\right)$, which provides sufficient control in time (see Lemma 6.3 below). As a result the remaining ingredient is a spatial translation (compactness) estimate on $\boldsymbol{u}_{h}$ in $L^{2}\left(0, T ; \boldsymbol{L}^{2}(\Omega)\right)$; cf. Lemma 2.3. However, our approximation space for $\boldsymbol{u}_{h}$ is only div-conforming. What saves us is the orthogonal structure of the finite element formulation and spaces. More specifically, we utilize the discrete orthogonal Hodge decomposition of Lemma 5.2 to write $\boldsymbol{u}_{h}=\operatorname{curl} \boldsymbol{\zeta}_{h}+\boldsymbol{z}_{h}$. Then, using the structure of the scheme, we observe that $\operatorname{curl} \zeta_{h}$ is not directly connected to the density $\varrho_{h}$, which in turn yields strong convergence of $\operatorname{curl} \zeta_{h}$ (Lemma 6.2). The required spatial translation estimate on $z_{h}$ is given by the following lemma.

Lemma 6.1. Given (6.1), define $\left\{\left(\boldsymbol{\zeta}_{h}, \boldsymbol{z}_{h}\right)\right\}_{h>0}$ in terms of the decomposition $\boldsymbol{u}_{h}(\cdot, t)=\operatorname{curl} \boldsymbol{\zeta}_{h}(t, \cdot)+\boldsymbol{z}_{h}(\cdot, t)$ with $\boldsymbol{\zeta}_{h}(\cdot, t) \in \boldsymbol{W}_{h}^{0, \perp}, \boldsymbol{z}_{h}(t, \cdot) \in \boldsymbol{V}_{h}^{0, \perp}$, for $t \in(0, T)$. Then, for any $\xi \in \mathbb{R}^{N}$,

$$
\left\|\boldsymbol{z}_{h}(t, \cdot)-\boldsymbol{z}_{h}(t, \cdot-\xi)\right\|_{L^{2}\left(0, T ; \boldsymbol{L}^{2}\left(\Omega_{\xi}\right)\right.}^{2} \leq C\left(|\xi|^{\frac{4-N}{2}}+|\xi|^{2}\right)\left\|\operatorname{div} \boldsymbol{z}_{h}\right\|_{L^{2}\left(0, T ; L^{2}(\Omega)\right)}^{2},
$$

where $\Omega_{\xi}=\{x \in \Omega: \operatorname{dist}(x, \partial \Omega)>\xi\}$.

Proof. For each $t \in(0, T)$ we know that $\boldsymbol{z}_{h}(t, \cdot) \in \boldsymbol{V}_{h}^{0, \perp}(\Omega)$, so Theorem A.1 can be applied to give

$$
\left\|\boldsymbol{z}_{h}(t, \cdot)-\boldsymbol{z}_{h}(t, \cdot-\xi)\right\|_{\boldsymbol{L}^{2}\left(\Omega_{\xi}\right)}^{2} \leq C\left(|\xi|^{\frac{4-N}{2}}+|\xi|^{2}\right)\left\|\operatorname{div} \boldsymbol{z}_{h}\right\|_{L^{2}(\Omega)}^{2},
$$

where $C>0$ is independent of $h, \xi, t$. We conclude by integrating over $(0, T)$.

Lemma 6.2. Given (6.1), define $\left\{\left(\boldsymbol{\zeta}_{h}, \boldsymbol{z}_{h}\right)\right\}_{h>0}$ in terms of the decomposition $\boldsymbol{u}_{h}(t, \cdot)=\operatorname{curl} \boldsymbol{\zeta}_{h}(t, \cdot)+\boldsymbol{z}_{h}(t, \cdot)$ with $\boldsymbol{\zeta}_{h}(t, \cdot) \in \boldsymbol{W}_{h}^{0, \perp}, \boldsymbol{z}_{h}(t, \cdot) \in \boldsymbol{V}_{h}^{0, \perp}, t \in(0, T)$. Then

$$
\boldsymbol{w}_{h} \stackrel{h \rightarrow 0}{\rightarrow}^{w}, \quad \operatorname{curl} \boldsymbol{\zeta}_{h} \stackrel{h \rightarrow 0}{\rightarrow}^{\operatorname{curl} \boldsymbol{\zeta}} \quad \text { in } L^{2}\left(0, T ; \boldsymbol{L}^{2}(\Omega)\right)
$$


Proof. Subtract the first equation of (3.2) with $\boldsymbol{v}_{h}=\operatorname{curl} \boldsymbol{\xi}_{h}^{m}$ from $\mu$ times the second equation of (3.2). Multiplying the result with $\Delta t$ and summing over all $m=1, \ldots, M$, yields

$$
\begin{gathered}
\int_{0}^{T} \int_{\Omega} \mu \operatorname{curl} \boldsymbol{\eta}_{h} \operatorname{curl} \boldsymbol{\zeta}_{h}-\mu \operatorname{curl} \boldsymbol{w}_{h} \operatorname{curl} \boldsymbol{\xi}_{h} d x d t \\
=\int_{0}^{T} \int_{\Omega} \mu \boldsymbol{w}_{h} \boldsymbol{\eta}_{h}-\boldsymbol{f}_{h} \operatorname{curl} \boldsymbol{\xi}_{h} d x d t
\end{gathered}
$$

for all $\boldsymbol{\eta}_{h}, \boldsymbol{\xi}_{h}$ that are piecewise constant in time with values in $\boldsymbol{W}_{h}(\Omega)$. Fixing $\boldsymbol{\eta}, \boldsymbol{\xi} \in C_{c}^{\infty}((0, T) \times \Omega)$, we use in (6.3) the test functions

$$
\begin{aligned}
& \boldsymbol{\xi}_{h}(t, \cdot)=\boldsymbol{\xi}_{h}^{m}(\cdot):=\frac{1}{\Delta t} \int_{t^{m-1}}^{t^{m}} \Pi_{h}^{W} \boldsymbol{\xi}(\cdot, s) d s, \quad t \in\left(t_{m-1}, t_{m}\right), m=1, \ldots, M . \\
& \boldsymbol{\eta}_{h}(t, \cdot)=\boldsymbol{\eta}_{h}^{m}(\cdot):=\frac{1}{\Delta t} \int_{t^{m-1}}^{t^{m}} \Pi_{h}^{W} \boldsymbol{\eta}(\cdot, s) d s, \quad t \in\left(t_{m-1}, t_{m}\right), m=1, \ldots, M .
\end{aligned}
$$

Due to Lemma 2.11 $\operatorname{curl} \boldsymbol{\xi}_{h} \rightarrow \operatorname{curl} \boldsymbol{\xi}$ and $\operatorname{curl} \boldsymbol{\eta}_{h} \rightarrow \operatorname{curl} \boldsymbol{\eta}$ in $L^{2}\left(0, T ; \boldsymbol{L}^{2}(\Omega)\right)$. As a consequence, keeping in mind (6.1), we let $h \rightarrow 0$ in (6.3) to obtain

$$
\begin{aligned}
& \int_{0}^{T} \int_{\Omega} \mu \operatorname{curl} \boldsymbol{\eta} \operatorname{curl} \boldsymbol{\zeta}-\mu \operatorname{curl} \boldsymbol{w} \operatorname{curl} \boldsymbol{\xi} d x d t \\
& \quad=\int_{0}^{T} \int_{\Omega} \mu \boldsymbol{w} \boldsymbol{\eta}-\boldsymbol{f} \operatorname{curl} \boldsymbol{\xi} d x d t, \quad \forall \boldsymbol{\eta}, \boldsymbol{\xi} \in \boldsymbol{C}_{c}^{\infty}((0, T) \times \Omega) .
\end{aligned}
$$

Since $\boldsymbol{C}_{c}^{\infty}((0, T) \times \Omega)$ is dense in $L^{2}\left(0, T ; \boldsymbol{W}_{0}^{\text {curl,2 }}(\Omega)\right)([17])$, we conclude that (6.4) holds for all $\boldsymbol{\eta}, \boldsymbol{\xi} \in L^{2}\left(0, T ; \boldsymbol{W}_{0}^{\text {curl, } 2}(\Omega)\right)$. Hence, taking $\boldsymbol{\eta}=\boldsymbol{w}, \boldsymbol{\xi}=\boldsymbol{\zeta}$ in (6.4),

$$
0=\int_{0}^{T} \int_{\Omega} \mu|\boldsymbol{w}|^{2}-\boldsymbol{f} \operatorname{curl} \boldsymbol{\zeta} d x d t
$$

Next, setting $\boldsymbol{\eta}_{h}=\boldsymbol{w}_{h}$ and $\boldsymbol{\xi}_{h}=\boldsymbol{\zeta}_{h}$ in (6.3), we observe that

$$
0=\int_{0}^{T} \int_{\Omega} \mu\left|\boldsymbol{w}_{h}\right|^{2}-\boldsymbol{f}_{h} \operatorname{curl} \boldsymbol{\zeta}_{h} d x d t .
$$

Letting $h \rightarrow 0$ and comparing the result with (6.5) reveals that

$$
\lim _{h \rightarrow 0} \int_{0}^{T} \int_{\Omega} \mu\left|\boldsymbol{w}_{h}\right|^{2} d x d t=\int_{0}^{T} \int_{\Omega} \mu|\boldsymbol{w}|^{2} d x d t,
$$

which implies the first part of 6.2):

$$
\boldsymbol{w}_{h} \rightarrow \boldsymbol{w} \quad \text { in } L^{2}\left(0, T ; \boldsymbol{L}^{2}(\Omega)\right) .
$$

To prove the second part of (6.2), we make use of $\boldsymbol{\eta}_{h}=\boldsymbol{\zeta}_{h}^{m}$ as a test function in the second equation of (3.2), sum the result over $m=1, \ldots, M$, and subsequently send $h$ to zero:

$$
\begin{aligned}
\lim _{h \rightarrow 0} \int_{0}^{T} \int_{\Omega}\left|\operatorname{curl} \boldsymbol{\zeta}_{h}\right|^{2} d x d t & =\lim _{h \rightarrow 0} \int_{0}^{T} \int_{\Omega} \boldsymbol{w}_{h} \boldsymbol{\zeta}_{h} d x d t \\
\stackrel{6.6 \mid}{=} & \int_{0}^{T} \int_{\Omega} \boldsymbol{w} \boldsymbol{\zeta} d x d t=\int_{0}^{T} \int_{\Omega}|\operatorname{curl} \boldsymbol{\zeta}|^{2} d x d t,
\end{aligned}
$$

where the last equality follows by setting $\boldsymbol{\eta}=\boldsymbol{\zeta}$ and $\boldsymbol{\xi}=0$ in (6.4). 
Lemma 6.3 (Convergence of $\left.\varrho_{h} \boldsymbol{u}_{h}\right)$. Given (6.1),

$$
\varrho_{h} \boldsymbol{u}_{h}{ }^{h \rightarrow 0} \varrho \boldsymbol{u} \quad \text { in the sense of distributions on }(0, T) \times \Omega \text {. }
$$

Proof. By virtue of Lemma 5.2, there exist sequences $\left\{\boldsymbol{\zeta}_{h}\right\}_{h>0},\left\{\boldsymbol{z}_{h}\right\}_{h>0}$ satisfying

$$
\begin{aligned}
& \boldsymbol{u}_{h}(\cdot, t)=\operatorname{curl} \boldsymbol{\zeta}_{h}(\cdot, t)+\boldsymbol{z}_{h}(\cdot, t), \\
& \boldsymbol{\zeta}_{h}(\cdot, t) \in \boldsymbol{W}_{h}^{0, \perp}, \quad \boldsymbol{z}_{h}(t, \cdot) \in \boldsymbol{V}_{h}^{0, \perp},
\end{aligned}
$$

for all $t \in(0, T)$. In Lemma 6.2 we proved that

$$
\operatorname{curl} \boldsymbol{\zeta}_{h} \rightarrow \operatorname{curl} \boldsymbol{\zeta} \text { in } L^{2}\left(0, T ; \boldsymbol{L}^{2}(\Omega)\right) .
$$

As a consequence, $\operatorname{curl} \zeta_{h} \varrho_{h} \rightarrow \operatorname{curl} \zeta \varrho$ in the sense of distributions.

It remains to prove that

$$
\varrho_{h} z_{h} \rightarrow \varrho z \quad \text { in the sense of distributions. }
$$

To this end, we adapt the proof of Lemma 2.3 to our specific discrete setting. We begin by introducing the regularized field $\boldsymbol{z}_{h}^{\epsilon}=\kappa_{\underset{(x)}{\epsilon}}^{\star} \boldsymbol{z}_{h}$, where $\kappa^{\epsilon}$ is a standard regularizing kernel and $\underset{(x)}{\star}$ denotes the convolution product (in $x$ ). Lemma 6.1 guarantees that

$$
\left\|\boldsymbol{z}_{h}^{\epsilon}-\boldsymbol{z}_{h}\right\|_{L^{2}\left(0, T ; \boldsymbol{L}^{2}(\Omega)\right)} \rightarrow 0 \text { as } \epsilon \rightarrow 0 \text {, uniformly in } h .
$$

In addition, for any $k$ and $p$, since $\boldsymbol{z}_{h}^{\epsilon} \in L^{2}\left(0, T ; \boldsymbol{W}^{k, p}(\Omega)\right)$ we have that $\boldsymbol{z}_{h}^{\epsilon} \stackrel{h \rightarrow 0}{0}^{\epsilon}$ in $L^{2}\left(0, T ; \boldsymbol{W}^{k, p}(\Omega)\right)$. Moreover, $\boldsymbol{z}^{\epsilon} \stackrel{\epsilon \rightarrow 0}{\rightarrow} \boldsymbol{z}$ in $L^{2}\left(0, T ; \boldsymbol{L}^{2}(\Omega)\right)$. Hence, by writing $\varrho_{h} \boldsymbol{z}_{h}=\varrho_{h}\left(\boldsymbol{z}_{h}-\boldsymbol{z}_{h}^{\epsilon}\right)+\varrho_{h} \boldsymbol{z}_{h}^{\epsilon}$ it suffices to prove $\varrho_{h} \boldsymbol{z}_{h}^{\epsilon}{ }^{h \rightarrow 0} \varrho^{\underline{\epsilon}} \boldsymbol{z}^{\epsilon}$ for each fixed $\epsilon>0$.

Next, let us introduce auxiliary functions $\boldsymbol{Z}_{h}^{\epsilon, m} \in \boldsymbol{V}_{h}, m=1, \ldots, M$, defined by

$$
\boldsymbol{Z}_{h}^{\epsilon, m}(x)=\Delta t \sum_{k=0}^{m} \boldsymbol{z}_{h}^{\epsilon, m}(x), \quad \boldsymbol{z}_{h}^{\epsilon, m}=\kappa^{\epsilon} \star \boldsymbol{z}_{h}^{m} .
$$

We extend $\left\{\boldsymbol{Z}_{h}^{\epsilon, m}\right\}_{m=1}^{M}$ to a function $\boldsymbol{Z}_{h}^{\epsilon}$ defined on $(-\Delta t, T] \times \Omega$ by setting

$$
\boldsymbol{Z}_{h}^{\epsilon}(t, \cdot)=\boldsymbol{Z}_{h}^{\epsilon, m}(\cdot), \quad t \in\left(t^{m-1}, t^{m}\right], \quad m=1, \ldots, M,
$$

and $\boldsymbol{Z}_{h}^{\epsilon}(t, \cdot)=\boldsymbol{Z}_{h}^{\epsilon, 0}$ for $t \in(-\Delta t, 0]$. In view of the regularity of $\boldsymbol{z}_{h}^{\epsilon}$,

$$
\boldsymbol{Z}_{h}^{\epsilon}(t, \cdot) \rightarrow \boldsymbol{Z}^{\epsilon}(t, \cdot)=\int_{0}^{t} \boldsymbol{z}^{\epsilon}(s, \cdot) d s \quad \text { in } C^{k}(\Omega) \text { for any } k \geq 0,
$$

uniformly in $t$ on $[0, T]$.

Now, we write

$$
\varrho_{h}^{m} \boldsymbol{z}_{h}^{\epsilon, m}=\frac{\varrho_{h}^{m} \boldsymbol{Z}_{h}^{\epsilon, m}-\varrho_{h}^{m-1} \boldsymbol{Z}_{h}^{\epsilon, m-1}}{\Delta t}-\boldsymbol{Z}_{h}^{\epsilon, m-1} \frac{\varrho_{h}^{m}-\varrho_{h}^{m-1}}{\Delta t},
$$

which alternatively can be written as

$$
\varrho_{h} \boldsymbol{z}_{h}^{\epsilon}=\frac{\partial}{\partial t} \Pi_{\mathcal{L}}\left(\varrho_{h} \boldsymbol{Z}_{h}^{\epsilon}\right)-\boldsymbol{Z}_{h}^{\epsilon}(\cdot-\Delta t, \cdot) \frac{\partial}{\partial t}\left(\Pi_{\mathcal{L}} \varrho_{h}\right),
$$

on $\left(t_{m-1}, t_{m}\right] \times \Omega, m=1, \ldots, M$. 
Fix $\phi \in C_{c}^{\infty}((0, T) \times \Omega)$. Summation by parts gives

$$
\begin{aligned}
\int_{0}^{T} & \int_{\Omega} \frac{\partial}{\partial t} \Pi_{\mathcal{L}}\left(\varrho_{h} \boldsymbol{Z}_{h}^{\epsilon}\right) \phi d x d t \\
& =-\int_{\Delta t}^{T} \int_{\Omega} \varrho_{h}(t-\Delta t, x) \boldsymbol{Z}_{h}^{\epsilon}(t-\Delta t, x) \frac{\partial}{\partial t}\left(\Pi_{\mathcal{L}} \phi_{h}\right) d x d t,
\end{aligned}
$$

where $\phi_{h}(t, \cdot)=\frac{1}{\Delta t} \int_{t^{m-1}}^{t^{m}} \phi(s, \cdot) d s$ for $t \in\left(t_{m-1}, t_{m}\right)$.

Thanks to (6.1) and (6.7), $\varrho_{h} \boldsymbol{Z}_{h}^{\epsilon} \stackrel{h \rightarrow 0}{\rightarrow} \varrho \boldsymbol{Z}^{\epsilon}$ in $L^{2 \gamma}\left(0, T ; L^{2 \gamma}(\Omega)\right) \cap L^{\infty}\left(0, T ; L^{\gamma}(\Omega)\right)$, and hence

$$
\frac{\partial}{\partial t} \Pi_{\mathcal{L}}\left(\varrho_{h} \boldsymbol{Z}_{h}^{\epsilon}\right) \stackrel{h \rightarrow 0}{\longrightarrow} \frac{\partial}{\partial t}\left(\varrho \boldsymbol{Z}^{\epsilon}\right) \quad \text { in the sense of distributions on }(0, T) \times \Omega .
$$

In addition, Lemma 5.6 tells us that $\frac{\partial}{\partial t}\left(\Pi_{\mathcal{L}} \varrho_{h}\right) \in_{b} L^{1}\left(0, T ; W^{-1,1}(\Omega)\right)$, and thus

$$
\boldsymbol{Z}_{h}^{\epsilon}(\cdot-\Delta t, \cdot) \frac{\partial}{\partial t}\left(\Pi_{\mathcal{L}} \varrho_{h}\right) \stackrel{h \rightarrow 0}{\rightarrow} \boldsymbol{Z}^{\epsilon} \frac{\partial}{\partial t} \varrho,
$$

in the sense of distributions on $(0, T) \times \Omega$.

Observing that $\varrho \boldsymbol{z}^{\epsilon}=\frac{d}{d t}\left(\varrho \boldsymbol{Z}^{\epsilon}\right)-\boldsymbol{Z}^{\epsilon} \frac{\partial \varrho}{\partial t}$, we conclude the proof.

Lemma 6.4 (Continuity equation). The limit pair $(\varrho, \boldsymbol{u})$ constructed in (6.1) is a weak solution of the continuity equation (1.1) in the sense of Definition 2.6.

Proof. Fix a test function $\phi \in C_{c}^{\infty}([0, T) \times \bar{\Omega})$, and introduce the piecewise constant approximations $\phi_{h}:=\Pi_{h}^{Q} \phi, \phi_{h}^{m}:=\Pi_{h}^{Q} \phi^{m}$, and $\phi^{m}:=\frac{1}{\Delta t} \int_{t^{m-1}}^{t^{m}} \phi(t, \cdot) d t$.

Let us employ $\phi_{h}^{m}$ as a test function in the continuity scheme (3.1) and sum over $m=1, \ldots, M$. The resulting equation reads

$$
\begin{aligned}
& \sum_{m=1}^{M} \Delta t \int_{\Omega} \frac{d}{d t}\left(\Pi_{\mathcal{L}} \varrho_{h}\right) \phi_{h}^{m} d x d t \\
& \quad=\sum_{\Gamma \in \Gamma_{h}^{I}} \sum_{m=1}^{M} \Delta t \int_{\Gamma}\left(\varrho_{-}^{m}\left(\boldsymbol{u}_{h}^{m} \cdot \nu\right)^{+}+\varrho_{+}^{m}\left(\boldsymbol{u}_{h}^{m} \cdot \nu\right)^{-}\right)\left[\phi_{h}^{m}\right]_{\Gamma} d S(x) .
\end{aligned}
$$

As in the proof of Lemma 5.6 we can rewrite this as

$$
\begin{aligned}
& \sum_{m=1}^{M} \Delta t \int_{\Omega} \frac{d}{d t}\left(\Pi_{\mathcal{L} \varrho_{h}}\right) \phi_{h}^{m} d x d t \\
& =\sum_{m=1}^{M} \Delta t \int_{\Omega} \varrho_{h}^{m} \boldsymbol{u}_{h}^{m} D \phi^{m} d x \\
& \quad+\sum_{E \in E_{h}} \sum_{m=1}^{M} \Delta t \int_{\partial E \backslash \partial \Omega}\left[\varrho_{h}^{m}\right]_{\partial E}\left(\boldsymbol{u}_{h}^{m} \cdot \nu\right)^{-}\left(\phi_{h}^{m}-\phi^{m}\right) d S(x) \\
& =\int_{0}^{T} \int_{\Omega} \varrho_{h} \boldsymbol{u}_{h} D \phi d x d t \\
& \quad+\sum_{E \in E_{h}} \int_{0}^{T} \int_{\partial E \backslash \partial \Omega}\left[\varrho_{h}\right]_{\partial E}\left(\boldsymbol{u}_{h} \cdot \nu\right)^{-}\left(\phi_{h}-\phi\right) d S(x) d t .
\end{aligned}
$$


Lemma 5.5 tells us that

$$
\left|\sum_{E \in E_{h}} \int_{0}^{T} \int_{\partial E \backslash \partial \Omega}\left[\varrho_{h}\right]_{\partial E}\left(\boldsymbol{u}_{h} \cdot \nu\right)^{-}\left(\phi_{h}-\phi\right) d S(x) d t\right| \leq C h^{\frac{1}{2}}\|D \phi\|_{L^{\infty}\left(0, T ; \boldsymbol{L}^{\infty}(\Omega)\right)} \text {. }
$$

In view of Lemma 6.3

$$
\lim _{h \rightarrow 0} \int_{0}^{T} \int_{\Omega} \varrho_{h} \boldsymbol{u}_{h} D \phi d x d t=\int_{0}^{T} \int_{\Omega} \varrho \boldsymbol{u} D \phi d x d t .
$$

Summation by parts gives

$$
\begin{aligned}
& \sum_{m=1}^{M} \Delta t \int_{\Omega} \frac{d}{d t}\left(\Pi_{\mathcal{L}} \varrho_{h}\right) \phi_{h}^{m} d x d t \\
& \quad=-\int_{\Delta t}^{T} \int_{\Omega} \varrho_{h}(t-\Delta t, x) \frac{\partial}{\partial t}\left(\Pi_{\mathcal{L}} \phi_{h}\right) d x d t-\int_{\Omega} \varrho_{h}^{0} \phi_{h}^{1} d x \\
& \quad \stackrel{h \rightarrow 0}{\rightarrow}-\int_{0}^{T} \int_{\Omega} \varrho \phi_{t} d x d t-\int_{\Omega} \varrho_{0} \phi(0, x) d x,
\end{aligned}
$$

where (6.1), together with the strong convergence $\varrho_{h}^{0} \stackrel{h \rightarrow 0}{\rightarrow} \varrho_{0}$, was used to pass to the limit. Summarizing, letting $h \rightarrow 0$ in (6.8) delivers the desired result (2.1)

6.2. Strong convergence of density approximations. The instrument used to establish strong convergence of the density approximations $\varrho_{h}$, is a weak continuity property of the quantity $P_{\text {eff }}\left(\varrho_{h}, \boldsymbol{u}_{h}\right)$ defined in (5.16). To derive this property we exploit our choice of numerical method and the boundary conditions; specifically, the finite element spaces, which are chosen such that (6.10) below holds.

Lemma 6.5 (Discrete effective viscous flux). Given the convergences in (6.1),

$$
\lim _{h \rightarrow 0} \int_{0}^{t} \int_{\Omega} P_{\mathrm{eff}}\left(\varrho_{h}, \boldsymbol{u}_{h}\right) \varrho_{h} d x d s=\int_{0}^{t} \int_{\Omega} \overline{P_{\mathrm{eff}}(\varrho, \boldsymbol{u})} \varrho d x d s, \quad \forall t \in(0, T) .
$$

Proof. For each $m=1, \ldots, M$, consider the problem

$$
\operatorname{div} \boldsymbol{v}_{h}^{m}=q_{h}^{m}-\frac{1}{|\Omega|} \int_{\Omega} q_{h}^{0} d x, \quad q_{h}^{m}:=\varrho_{h}^{m}-\frac{1}{\Delta t} \int_{t^{m-1}}^{t^{m}} \Pi_{h}^{Q} \varrho d t,
$$

where $q_{h}^{0}=\varrho_{h}^{0}-\varrho_{0}$. Observe that $\int_{\Omega} q_{h}^{m} d x=0$. Indeed, using the continuity scheme (3.1) and the continuity equation satisfied by the limit $\varrho$; cf. Lemma 6.4

$$
\int_{\Omega} \varrho_{h} d x=\int_{\Omega} \varrho_{h}^{0} d x, \quad \int_{\Omega} \Pi_{h}^{Q} \varrho d x=\int_{\Omega} \varrho d x=\int_{\Omega} \varrho_{0} d x .
$$

Thus, there exists a unique solution $\boldsymbol{v}_{h}^{m} \in \boldsymbol{V}_{h}^{0, \perp}$ of (6.9). We denote by $\boldsymbol{v}_{h}(t, \cdot)$, $q_{h}(t, \cdot)$ the usual "piecewise constant" extensions of $\left\{v_{h}^{m}\right\}_{m=1}^{M},\left\{q_{h}^{m}\right\}_{m=1}^{M}$ to $(0, T)$.

Utilizing $\boldsymbol{v}_{h}^{m}$ as a test function, the velocity scheme (3.2) reads

$$
\int_{\Omega} P_{\mathrm{eff}}\left(\varrho_{h}^{m}, \boldsymbol{u}_{h}^{m}\right) q_{h}^{m} d x=\frac{1}{|\Omega|} \int_{\Omega} q_{h}^{0} d x\left(\int_{\Omega} P_{\mathrm{eff}}\left(\varrho_{h}^{m}, \boldsymbol{u}_{h}^{m}\right) d x\right)-\int_{\Omega} \boldsymbol{f}_{h}^{m} \boldsymbol{v}_{h}^{m} d x
$$


Multiplying by $\Delta t$, summing over $m$, and using the definition of $q_{h}^{m}$, we arrive at

$$
\begin{aligned}
\int_{0}^{t} \int_{\Omega} P_{\mathrm{eff}}\left(\varrho_{h}, \boldsymbol{u}_{h}\right)\left(\varrho_{h}-\varrho\right) d x d s= & \frac{1}{|\Omega|} \int_{\Omega} q_{h}^{0} d x\left(\int_{0}^{t} \int_{\Omega} P_{\mathrm{eff}}\left(\varrho_{h}, \boldsymbol{u}_{h}\right) d x d t\right) \\
& -\int_{0}^{t} \int_{\Omega} \boldsymbol{f}_{h} \boldsymbol{v}_{h} d x d s
\end{aligned}
$$

for any $t \in(0, T)$.

In view of Theorem A.1, we have that $\boldsymbol{v}_{h} \rightarrow 0$ in $L^{2}\left(0, T ; \boldsymbol{L}^{2}(\Omega)\right)$. Since $\boldsymbol{f}_{h} \rightarrow \boldsymbol{f}$ in $L^{2}((0, T) \times \Omega)$ and $\int_{\Omega} q_{h}^{0} d x \rightarrow 0$, we conclude the desired result

$$
\lim _{h \rightarrow 0} \int_{0}^{t} \int_{\Omega} P_{\text {eff }}\left(\varrho_{h}, \boldsymbol{u}_{h}\right)\left(\varrho_{h}-\varrho\right) d x d s=0 .
$$

We are now in a position to infer the sought-after strong convergence of the density approximations.

Lemma 6.6 (Strong convergence of $\varrho_{h}$ ). Suppose that (6.1) holds. Then, passing to a subsequence if necessary,

$$
\varrho_{h} \rightarrow \varrho \quad \text { a.e. in }(0, T) \times \Omega \text {. }
$$

Proof. In view of Lemma 6.4 the limit $(\varrho, \boldsymbol{u})$ is a weak solution of the continuity equation and hence, by Lemma 2.9, also a renormalized solution. In particular,

$$
(\varrho \log \varrho)_{t}+\operatorname{div}((\varrho \log \varrho) \boldsymbol{u})=-\varrho \operatorname{div} \boldsymbol{u} \text { in the weak sense on }[0, T) \times \bar{\Omega} \text {. }
$$

Since $t \mapsto \varrho \log \varrho$ is continuous with values in some Lebesgue space equipped with the weak topology, we can use this equation to obtain for any $t>0$,

$$
\int_{\Omega}(\varrho \log \varrho)(t) d x-\int_{\Omega} \varrho_{0} \log \varrho_{0} d x=-\int_{0}^{t} \int_{\Omega} \varrho \operatorname{div} \boldsymbol{u} d x d s .
$$

Next, we specify $\phi_{h} \equiv 1$ as a test function in the renormalized scheme (5.1), multiply by $\Delta t$, and sum the result over $m$. Making use of the convexity of $z \log z$, we infer for any $m=1, \ldots, M$,

$$
\int_{\Omega} \varrho_{h}^{m} \log \varrho_{h}^{m} d x-\int_{\Omega} \varrho_{h}^{0} \log \varrho_{h}^{0} d x \leq-\sum_{k=1}^{m} \Delta t \int_{\Omega} \varrho_{h}^{k} \operatorname{div} \boldsymbol{u}_{h}^{k} d x
$$

In view of the convergences stated at the beginning of this section and strong convergence of the initial data, we can send $h \rightarrow 0$ in (6.12) to obtain

$$
\int_{\Omega}(\overline{\varrho \log \varrho})(t) d x-\int_{\Omega} \varrho_{0} \log \varrho_{0} d x \leq-\int_{0}^{t} \int_{\Omega} \overline{\varrho \operatorname{div} \boldsymbol{u}} d x d s .
$$

Subtracting (6.11) from (6.13) gives

$$
\int_{\Omega}(\overline{\varrho \log \varrho}-\varrho \log \varrho)(t) d x \leq-\int_{0}^{t} \int_{\Omega} \overline{\varrho \operatorname{div} \boldsymbol{u}}-\varrho \operatorname{div} \boldsymbol{u} d x d s,
$$

for any $t \in(0, T)$. Lemma 6.5 tells us that

$$
\int_{0}^{t} \int_{\Omega} \overline{\varrho \operatorname{div} \boldsymbol{u}}-\varrho \operatorname{div} \boldsymbol{u} d x d s=\frac{a}{\mu+\lambda} \int_{0}^{t} \int_{\Omega} \overline{\varrho^{\gamma+1}}-\overline{\varrho^{\gamma}} \varrho d x d s \geq 0,
$$

where the last inequality follows as in [14, 22, so the following relation holds:

$$
\overline{\varrho \log \varrho}=\varrho \log \varrho \quad \text { a.e. in }(0, T) \times \Omega .
$$

Now an application of Lemma 2.1 brings the proof to an end. 


\subsection{Velocity scheme.}

Lemma 6.7 (Velocity equation). The limit triple $(\boldsymbol{w}, \boldsymbol{u}, \varrho)$ constructed in (6.1) is a weak solution of the velocity equation (1.2) in the sense of (2.3).

Proof. Fix $(\boldsymbol{v}, \boldsymbol{\eta}) \in \boldsymbol{C}_{c}^{\infty}((0, T) \times \Omega)$, and introduce the projections $\boldsymbol{v}_{h}=\Pi_{h}^{V} \boldsymbol{v}$, $\boldsymbol{\eta}_{h}=\Pi_{h}^{W} \boldsymbol{\eta}$ and $\boldsymbol{v}_{h}^{m}=\frac{1}{\Delta t} \int_{t^{m-1}}^{t^{m}} \boldsymbol{v}_{h} d t, \boldsymbol{\eta}_{h}^{m}=\frac{1}{\Delta t} \int_{t^{m-1}}^{t^{m}} \boldsymbol{\eta}_{h} d t$.

Utilizing $\boldsymbol{v}_{h}^{m}$ and $\boldsymbol{\eta}_{h}^{m}$ as test functions in the velocity scheme (3.2), multiplying by $\Delta t$, and summing the result over $m$, we gather

$$
\begin{aligned}
& \int_{0}^{T} \int_{\Omega} \mu \operatorname{curl} \boldsymbol{w}_{h} \boldsymbol{v}_{h}+\left[(\mu+\lambda) \operatorname{div} \boldsymbol{u}_{h}-p\left(\varrho_{h}\right)\right] \operatorname{div} \boldsymbol{v}_{h} d x d t=\int_{0}^{T} \int_{\Omega} \boldsymbol{f}_{h} \boldsymbol{v}_{h} d x d t, \\
& \int_{0}^{T} \int_{\Omega} \boldsymbol{w}_{h} \boldsymbol{\eta}_{h}-\boldsymbol{u}_{h} \operatorname{curl} \boldsymbol{\eta}_{h} d x d t=0 .
\end{aligned}
$$

From Lemma 2.11 we have $\boldsymbol{v}_{h} \stackrel{h \rightarrow 0}{\rightarrow} \boldsymbol{v}$ in $L^{2}\left(0, T ; \boldsymbol{W}_{0}^{\text {div, }}(\Omega)\right)$ and $\boldsymbol{\eta}_{h} \stackrel{h \rightarrow 0}{\rightarrow} \boldsymbol{\eta}$ in $L^{2}\left(0, T ; \boldsymbol{W}_{0}^{\text {curl, } 2}(\Omega)\right)$. Furthermore, by Lemma 6.6 and the first part of (6.1), $p\left(\varrho_{h}\right) \stackrel{h \rightarrow 0}{\rightarrow} p(\varrho)$ in $L^{2}((0, T) \times \Omega)$. Hence, we can send $h \rightarrow 0$ in (6.14) to obtain that the limit $(\boldsymbol{w}, \boldsymbol{u}, \varrho)$ constructed in (6.1) satisfies (2.3) for all test functions $(\boldsymbol{v}, \boldsymbol{\eta}) \in \boldsymbol{C}_{c}^{\infty}((0, T) \times \Omega)$. Since $\boldsymbol{C}_{c}^{\infty}((0, T) \times \Omega)$ is dense in both $L^{2}\left(0, T ; \boldsymbol{W}_{0}^{\mathrm{div}, 2}(\Omega)\right)$ and $L^{2}\left(0, T ; \boldsymbol{W}_{0}^{\text {curl, } 2}(\Omega)\right)$ [17, this concludes the proof.

\section{Appendix A. Compactness of functions in $V_{h}^{0, \perp}(\Omega)$}

In this appendix we prove that discrete weakly curl-free approximations in $\boldsymbol{V}^{0, \perp}(\Omega)$ with $L^{2}$ bounded divergence possess an $\boldsymbol{L}^{2}$ space translation estimate, which was previously needed (in Lemma 6.3) to conclude the weak convergence of the product $\varrho_{h} \boldsymbol{z}_{h}$ to the product of the corresponding weak limits $\varrho z$. As part of the proof, in Lemma A.3 we show that if a sequence $\left\{\boldsymbol{z}_{h}\right\}_{h>0}$ belongs to $\boldsymbol{V}_{h}^{0, \perp}(\Omega)$ and additionally satisfies $\operatorname{div} \boldsymbol{z}_{h} \in_{b} L^{2}(\Omega)$, then $\left\{\operatorname{curl} \boldsymbol{z}_{h}\right\}_{h>0}$ and $\left\{\operatorname{div} \boldsymbol{z}_{h}\right\}_{h>0}$ are actually compact in $W^{-1,2}(\Omega)$. Thus, strong $L^{2}(\Omega)$ convergence of a subsequence of $\left\{\boldsymbol{z}_{h}\right\}_{h>0}$ follows directly from the div-curl lemma. However, this is not sufficient to conclude the sought-after convergence of $\varrho_{h} z_{h}$ (cf. Subsection 6.1). The problem is a lack of temporal control of the velocity approximations $\boldsymbol{z}_{h}$.

The main result in this appendix is:

Theorem A.1. Given $\boldsymbol{z}_{h} \in \boldsymbol{V}_{h}^{0, \perp}$, there exists a constant $C>0$, depending only on $\Omega$ and the shape regularity of $E_{h}$, such that for every vector $\xi \in \mathbb{R}^{N}$,

$$
\left\|\boldsymbol{z}_{h}(\cdot)-\boldsymbol{z}_{h}(\cdot-\xi)\right\|_{\boldsymbol{L}^{2}\left(\Omega_{\xi}\right)} \leq C\left(|\xi|^{\frac{4-N}{2}}+|\xi|^{2}\right)^{\frac{1}{2}}\left\|\operatorname{div} \boldsymbol{z}_{h}\right\|_{L^{2}(\Omega)},
$$

where $\Omega_{\xi}=\{x \in \Omega: \operatorname{dist}(x, \partial \Omega)>\xi\}$.

In the proof of Theorem A.1 the essential ingredient is a bound on the tangential jumps $\left[\boldsymbol{z}_{h} \times \nu\right]_{\Gamma}$ across element faces. We will derive this bound in Section A.2. The derivation makes use of a technical result (Lemma A.3) which we derive first (in the ensuing section). The proof of Theorem A.1 is given at the end of the appendix (Section A.3). 


\section{A.1. Negative space compactness of the curl.}

Theorem A.2. Consider the mixed Laplace-type problem

$$
\begin{aligned}
& \operatorname{curl} \boldsymbol{w}-D \operatorname{div} \boldsymbol{u}=\boldsymbol{f}, \boldsymbol{w}=\operatorname{curl} \boldsymbol{u} \quad \text { in } \Omega, \\
& \boldsymbol{u} \cdot \nu=0, \boldsymbol{w} \times \nu=0 \quad \text { on } \partial \Omega,
\end{aligned}
$$

where we assume $\boldsymbol{f} \in \boldsymbol{L}^{2}(\Omega)$. There exists a pair

$$
(\boldsymbol{w}, \boldsymbol{u}) \in \boldsymbol{W}_{0}^{\mathrm{curl}, 2}(\Omega) \times \boldsymbol{W}_{0}^{\operatorname{div}, 2}(\Omega),
$$

satisfying (A.2) in the weak sense. Moreover, there exists a pair

$$
\left(\boldsymbol{w}_{h}, \boldsymbol{u}_{h}\right) \in \boldsymbol{W}_{h}(\Omega) \times \boldsymbol{V}_{h}(\Omega),
$$

satisfying the corresponding mixed finite element formulation of (A.2). Finally, the following error estimate holds:

$$
\left\|\boldsymbol{w}-\boldsymbol{w}_{h}\right\|_{\boldsymbol{L}^{2}(\Omega)}+\left\|\boldsymbol{u}-\boldsymbol{u}_{h}\right\|_{\boldsymbol{V}_{h}} \leq C h^{s}\|\boldsymbol{f}\|_{\boldsymbol{L}^{2}(\Omega)},
$$

where the convergence rate $s \in[1 / 2,1)$ depends on the regularity of $\partial \Omega$. If $\partial \Omega$ is Lipschitz and convex, A.3 holds with $s=1$.

Proof. Consult Theorem 7.9 in 2, for example.

Lemma A.3. Let $\left\{\boldsymbol{z}_{h}\right\}_{h>0}$ be a sequence in $\boldsymbol{V}_{h}^{0, \perp}$ for which $\left\|\operatorname{div} \boldsymbol{z}_{h}\right\|_{L^{2}(\Omega)} \leq C$, where the constant $C>0$ is independent of $h$. Then

$$
\left\|\operatorname{curl} \boldsymbol{z}_{h}\right\|_{\boldsymbol{W}^{-1,2}(\Omega)} \leq C h\left\|\operatorname{div} \boldsymbol{z}_{h}\right\|_{L^{2}(\Omega)}
$$

for some constant $C$ independent of $h$.

Proof. To prove this lemma, we will use the mixed system (A.2) to define a new operator. To motivate the construction, consider the problem

$$
-\Delta \boldsymbol{\theta}=\operatorname{curl} \phi \quad \text { in } \Omega, \quad \boldsymbol{\theta} \cdot \nu=0, \operatorname{curl} \boldsymbol{\theta} \times \nu=0 \quad \text { on } \partial \Omega,
$$

for some given $\phi \in \boldsymbol{W}_{0}^{\text {curl,2 }}(\Omega)$. By utilizing $D \Delta^{-1} \operatorname{div} \boldsymbol{\theta}$ as a test function in the weak formulation of (A.4), where $\Delta^{-1}$ is the inverse Neumann Laplace, it is easily seen that the weak solution $\boldsymbol{\theta}$ of the system (A.4) is divergence free. Furthermore, we can set $\boldsymbol{w}=\operatorname{curl} \boldsymbol{\theta}$ and integrate by parts to conclude that the pair $(\boldsymbol{w}, \boldsymbol{\theta})$ is also the unique weak solution of the mixed Laplace system (A.2) with $f=\operatorname{curl} \phi$.

Now we define a new operator $\Pi^{h}: \boldsymbol{W}_{0}^{\text {curl,2 }} \rightarrow \boldsymbol{W}_{h}$ as the unique function $\Pi^{h} \boldsymbol{\phi} \in \boldsymbol{W}_{h}$ satisfying the finite element formulation

$$
\begin{aligned}
\int_{\Omega} \operatorname{curl}\left(\Pi^{h} \boldsymbol{\phi}\right) \boldsymbol{v}_{h}+\operatorname{div} \boldsymbol{\theta}_{h} \operatorname{div} \boldsymbol{v}_{h} d x & =\int_{\Omega} \operatorname{curl} \boldsymbol{\phi} \boldsymbol{v}_{h} d x, \quad \forall \boldsymbol{v}_{h} \in \boldsymbol{V}_{h}, \\
\int_{\Omega} \operatorname{curl} \boldsymbol{\eta}_{h} \boldsymbol{\theta}_{h} d x & =\int_{\Omega}\left(\Pi^{h} \boldsymbol{\phi}\right) \boldsymbol{\eta}_{h} d x, \quad \forall \boldsymbol{\eta}_{h} \in \boldsymbol{W}_{h} .
\end{aligned}
$$

The existence of such a function $\Pi^{h} \phi$ is given by Theorem A.2 Using the fact that $\operatorname{div} \boldsymbol{\theta}=0$, the error estimate (A.3) yields

(A.6) $\left\|\Pi^{h} \boldsymbol{\phi}-\operatorname{curl} \boldsymbol{\theta}\right\|_{\boldsymbol{L}^{2}(\Omega)}+\left\|\boldsymbol{\theta}_{h}-\boldsymbol{\theta}\right\|_{\boldsymbol{L}^{2}(\Omega)}+\left\|\operatorname{div} \boldsymbol{\theta}_{h}\right\|_{L^{2}(\Omega)} \leq C h^{s}\|\operatorname{curl} \boldsymbol{\phi}\|_{\boldsymbol{L}^{2}(\Omega)}$. 
Let $\boldsymbol{z}_{h}$ be as stated in the lemma. Since $\boldsymbol{z}_{h}$ is orthogonal to functions in $\boldsymbol{W}_{h}$,

$$
\begin{aligned}
\left\|\operatorname{curl} \boldsymbol{z}_{h}\right\|_{\boldsymbol{W}^{-1,2}(\Omega)} & =\sup _{\boldsymbol{\phi} \in \boldsymbol{W}_{0}^{1,2}} \frac{\left|\int_{\Omega} \boldsymbol{z}_{h} \operatorname{curl} \boldsymbol{\phi} d x\right|}{\|\boldsymbol{\phi}\|_{\boldsymbol{W}^{1,2}(\Omega)}} \\
& =\sup _{\boldsymbol{\phi} \in \boldsymbol{W}_{0}^{1,2}} \frac{\left|\int_{\Omega} \boldsymbol{z}_{h} \operatorname{curl}\left(\boldsymbol{\phi}-\Pi^{h} \boldsymbol{\phi}\right) d x\right|}{\|\boldsymbol{\phi}\|_{\boldsymbol{W}^{1,2}(\Omega)}} \\
& \stackrel{\text { A.5D }}{=} \sup _{\boldsymbol{\phi} \in \boldsymbol{W}_{0}^{1,2}} \frac{\left|\int_{\Omega} \operatorname{div} \boldsymbol{z}_{h} \operatorname{div} \boldsymbol{\theta}_{h} d x\right|}{\|\boldsymbol{\phi}\|_{\boldsymbol{W}^{1,2}(\Omega)}} \\
& \leq C h\left\|\operatorname{div} \boldsymbol{z}_{h}\right\|_{L^{2}(\Omega)},
\end{aligned}
$$

where we have used A.6 to derive the last inequality, specifically the estimate

$$
\left\|\operatorname{div} \boldsymbol{\theta}_{h}\right\|_{L^{2}(\Omega)} \leq C h\|\operatorname{curl} \phi\|_{L^{2}(\Omega)} \leq C h\|\phi\|_{W^{1,2}(\Omega)} .
$$

This concludes the proof.

\section{A.2. Tangential jumps.}

Lemma A.4. Fix any $\Gamma \in \Gamma_{h}$. There exists a continuous piecewise linear (scalar) function $\phi$ on $\Gamma$ such that $\left.\phi\right|_{\partial \Gamma}=0,|\phi(x)| \leq 1 \forall x \in \Gamma$, and

$$
\int_{\Gamma} \phi f d x=\frac{1}{N} \int_{\Gamma} f d x, \quad \forall f \in \mathbb{P}^{1}(\Gamma),
$$

where $N$ is the spatial dimension.

Proof. Let $b$ denote the barycentric middle point with respect to the vertices of $\Gamma$. Let $T_{h}$ be the triangulation of $\Gamma$ obtained by setting $b$ as a vertex in addition to the vertices of $\Gamma$. On $T_{h}$ let $L_{h}(\Gamma)$ denote the standard finite element space of continuous piecewise linear functions. Any function $\phi_{h} \in L_{h}(\Gamma)$ is uniquely determined by its value at the vertices.

Now the relevant test function $\phi \in L_{h}(\Gamma)$ is obtained by requiring

$$
\phi(b)=1 \text { and } \phi\left(v_{i}\right)=0 \text {, for all vertices } v_{i} \text { at } \partial \Gamma \text {. }
$$

By a direct calculation it can be verified that $\phi$ satisfies (A.7).

Lemma A.5. Given $\boldsymbol{z}_{h} \in \boldsymbol{V}_{h}^{0, \perp}$, there exists a constant $C>0$, independent of $h$, such that

$$
\left|\int_{\Gamma}\left[\boldsymbol{z}_{h} \times \nu\right] d S(x)\right| \leq C h^{\frac{N}{2}}\left\|\operatorname{div} \boldsymbol{z}_{h}\right\|_{L^{2}(\Omega)}, \quad \forall \Gamma \in \Gamma_{h}
$$

and

$$
\sum_{\Gamma \in \Gamma_{h}^{I}}\left|\int_{\Gamma}\left[\boldsymbol{z}_{h} \times \nu\right] d S(x)\right|^{2} \leq C|\Omega|^{\frac{1}{2}} h^{\frac{N}{2}}\left\|\operatorname{div} \boldsymbol{z}_{h}\right\|_{L^{2}(\Omega)}^{2} .
$$

Proof. Let $\phi \in \boldsymbol{W}_{0}^{1,2}(\Omega)$. By virtue of Lemma A.3.

$$
\left|\int_{\Omega} \boldsymbol{z}_{h} \operatorname{curl} \boldsymbol{\phi} d x\right| \leq C h\|\boldsymbol{\phi}\|_{\boldsymbol{W}^{1,2}(\Omega)}\left\|\operatorname{div} \boldsymbol{z}_{h}\right\|_{L^{2}(\Omega)} .
$$

An integration by parts (2.4), keeping in mind curl $\left.\boldsymbol{z}_{h}\right|_{E}=0$ for all $E \in E_{h}$, yields

$$
\sum_{\Gamma \in \Gamma_{h}^{I}} \int_{\Gamma} \phi\left[\boldsymbol{z}_{h} \times \nu\right] d S(x)=\int_{\Omega} \boldsymbol{z}_{h} \operatorname{curl} \boldsymbol{\phi} d x
$$


and so

$$
\left|\sum_{\Gamma \in \Gamma_{h}^{I}} \int_{\Gamma} \boldsymbol{\phi}\left[\boldsymbol{z}_{h} \times \nu\right] d S(x)\right| \leq C h\|\boldsymbol{\phi}\|_{\boldsymbol{W}^{1,2}(\Omega)}\left\|\operatorname{div} \boldsymbol{z}_{h}\right\|_{L^{2}(\Omega)} .
$$

The bound (A.10) serves as the starting point for proving (A.8) and (A.9); the remaining objective is to construct a suitable test function $\phi$. Fix $\Gamma \in \Gamma_{h}^{I}$. Let $E_{-}, E_{+}$denote the two elements in $E_{h}$ sharing the edge/face $\Gamma$, where $E_{-}, E_{+}$are chosen so that $\nu$ points from $E_{-}$to $E_{+}$. In view of Lemma A.4 we can choose a continuous piecewise linear (scalar) function $\tilde{\phi}$ on $\Gamma$ such that

$$
\int_{\Gamma} \tilde{\phi} f d x=\frac{1}{N} \int_{\Gamma} f d x, \quad \forall f \in \mathbb{P}_{1}(\Gamma) .
$$

Denote by $\phi_{\partial E}$ the zero extension of $\tilde{\phi}$ to $\left(\partial E_{-} \backslash \Gamma\right) \bigcup\left(\partial E_{+} \backslash \Gamma\right)$, and fix a piecewise affine function $\phi_{E}$ on $E_{-} \cup E_{+}$such that $\left.\phi_{E}\right|_{\partial E_{+} \cup \partial E_{-}}=\phi_{\partial E}$. Clearly, $\phi_{E}$ can be chosen such that

$$
\left|D \phi_{E}\right| \leq C h^{-1} \text { in the interior of } E_{-} \cup E_{+} .
$$

Finally, let $\phi_{\Gamma}$ denote the extension by zero of $\phi_{E}$ to all of $\Omega$.

The function $\phi_{\Gamma}$ possesses the following properties: $\phi_{\Gamma} \in W_{0}^{1,2}(\Omega),\left.\phi_{\Gamma}\right|_{\tilde{\Gamma}}=0$ for all $\tilde{\Gamma} \in \Gamma_{h}$ such that $\tilde{\Gamma} \neq \Gamma$, and

$$
\left\|\phi_{\Gamma}\right\|_{W^{1,2}(\Omega)}^{2}=\left\|\left.\phi_{\Gamma}\right|_{E_{-}}\right\|_{W^{1,2}\left(E_{-}\right)}^{2}+\left\|\left.\phi_{\Gamma}\right|_{E_{+}}\right\|_{W^{1,2}\left(E_{+}\right)}^{2} \leq C h^{N}\left(1+h^{-2}\right) .
$$

If $N=2$ (curl is scalar), then we opt for $\phi=\phi_{\Gamma}$ in (A.10) to obtain

$$
\left|\int_{\Gamma}\left[\boldsymbol{z}_{h} \times \nu\right] d S(x)\right| \leq C h^{\frac{N}{2}}\left\|\operatorname{div} \boldsymbol{z}_{h}\right\|_{L^{2}(\Omega)} .
$$

If $N=3$, A.11 still holds. Indeed, to conclude we can in A.10 successively take $\phi=\left[\phi_{\Gamma}, 0,0\right]^{T}, \phi=\left[0, \phi_{\Gamma}, 0\right]^{T}$, and $\phi=\left[0,0, \phi_{\Gamma}\right]^{T}$. Since $\Gamma \in \Gamma_{h}$ was arbitrary, this concludes the proof of (A.8).

To establish (A.9), we introduce the test function

$$
\phi_{\Gamma}=\phi_{\Gamma} \int_{\Gamma}\left[\boldsymbol{z}_{h} \times \nu\right] d S(x), \quad \forall \Gamma \in \Gamma_{h}^{I},
$$

where $\phi_{\Gamma}$ is constructed as above with the additional requirement that

$$
\operatorname{supp} \phi_{\Gamma} \bigcap \operatorname{supp} \phi_{\tilde{\Gamma}}=\emptyset, \quad \forall \tilde{\Gamma} \neq \Gamma .
$$

We have

$$
\sup _{x \in \Omega}\left|D \phi_{\Gamma}\right| \leq C h^{-1}\left|\int_{\Gamma}\left[\boldsymbol{z}_{h} \times \nu\right] d S(x)\right|, \quad \forall \Gamma \in \Gamma_{h}^{I},
$$

and, for each $\Gamma \in \Gamma_{h}$,

$$
\int_{\Gamma} \phi_{\Gamma} f d S(x)=\frac{1}{N}\left(\int _ { \Gamma } [ \boldsymbol { z } _ { h } \times \nu ] d S ( x ) \left(\int_{\Gamma} f d S(x), \quad \forall f \in \mathbb{P}^{1}(\Gamma) .\right.\right.
$$

Finally, we set $\phi:=\sum_{\Gamma \in \Gamma_{h}^{I}} \phi_{\Gamma}$; this function satisfies $\phi \in \boldsymbol{W}_{0}^{1,2}(\Omega)$ and

$$
\sup _{x \in \Omega}|D \phi| \leq C h^{-1} \max _{\Gamma \in \Gamma_{h}^{I}}\left|\int_{\Gamma}\left[\boldsymbol{z}_{h} \times \nu\right] d S(x)\right| \leq C h^{\frac{N-2}{2}}\left\|\operatorname{div} \boldsymbol{z}_{h}\right\|_{L^{2}(\Omega)} .
$$


The last inequality follows from (A.8). A direct calculation gives

$$
\|D \phi\|_{L^{2}(\Omega)} \leq C h^{\frac{N-2}{2}}|\Omega|^{\frac{1}{2}}\left\|\operatorname{div} \boldsymbol{z}_{h}\right\|_{L^{2}(\Omega)} .
$$

Setting $\phi$ as a test function in A.10 immediately gives the estimate

$$
\sum_{\Gamma \in \Gamma_{h}^{I}}\left|\int_{\Gamma}\left[\boldsymbol{z}_{h} \times \nu\right] d S(x)\right|^{2} \leq C h^{\frac{N}{2}}|\Omega|^{\frac{1}{2}}\left\|\operatorname{div} \boldsymbol{z}_{h}\right\|_{L^{2}(\Omega)}^{2},
$$

which is (A.9).

A.3. Proof of Theorem A.1. The proof is inspired by Brenner's work [6] on Poincaré-Friedrich inequalities for piecewise $H^{1}$ vector fields. The basic idea is to project the relevant function into the Crouzeix-Raviart element space and then use the standard translation estimate satisfied by functions in this space (cf. Stummel [27]). Then, since the relevant function is discrete weakly curl free, we can use Lemma A.5 to suitably bound the projection error.

The Crouzeix-Raviart element space $\boldsymbol{R}_{h}(\Omega)$ is defined as follows:

$$
\boldsymbol{R}_{h}(\Omega)=\left\{\boldsymbol{v} \in \boldsymbol{L}^{2}(\Omega) ;\left.\boldsymbol{v}\right|_{E} \in \mathbb{P}_{1}^{N}(E), \forall E \in E_{h} ; \int_{\Gamma}[\boldsymbol{v}]_{\Gamma} d S(x)=0, \forall \Gamma \in \Gamma_{h}\right\} .
$$

The degrees of freedom of $\boldsymbol{R}_{h}(\Omega)$ are the average values $\int_{\Gamma} \boldsymbol{v}_{h} d S\left(x, \forall \Gamma \in \Gamma_{h}\right.$.

Let us introduce an interpolation operator $\Pi_{h}^{R}: \boldsymbol{V}_{h} \rightarrow \boldsymbol{R}_{h}$ by specifying

$$
\frac{1}{|\Gamma|} \int_{\Gamma} \Pi_{h}^{R} \boldsymbol{z}_{h} d S(x)=\frac{1}{|\Gamma|} \int_{\Gamma}\left\{\boldsymbol{z}_{h}\right\} d S(x), \quad \forall \Gamma \in \Gamma_{h}^{I},
$$

where $\{\cdot\}$ denotes the average of the traces from the two sides of $\Gamma$. According to Brenner [6], we have the following error estimate:

$$
\begin{aligned}
\| \boldsymbol{z}_{h} & -\Pi_{h}^{R} \boldsymbol{z}_{h} \|_{\boldsymbol{L}^{2}(\Omega)}^{2} \\
& \leq C\left(\sum_{E \in E_{h}} h^{2}\left\|D \boldsymbol{z}_{h}\right\|_{\boldsymbol{L}^{2}(\Omega)}^{2}+\sum_{\Gamma \in \Gamma_{h}^{I}} h^{2-N}\left|\int_{\Gamma}\left[\boldsymbol{z}_{h}\right] d S(x)\right|^{2}\right) .
\end{aligned}
$$

By a standard decomposition of vector fields,

$$
\left[\boldsymbol{z}_{h}\right]_{\Gamma}=\left[\left(\boldsymbol{z}_{h} \cdot \nu\right) \nu\right]_{\Gamma}-\left[\left(\boldsymbol{z}_{h} \times \nu\right) \times \nu\right]_{\Gamma}=-\left[\left(\boldsymbol{z}_{h} \times \nu\right) \times \nu\right]_{\Gamma},
$$

where the last equality follows for the reason that $\left[\boldsymbol{z}_{h} \cdot \nu\right]_{\Gamma}=0$ for all $\Gamma \in \Gamma_{h}^{I}$. To have the above decomposition well defined in two dimensions, we set $\left(\boldsymbol{z}_{h} \times \nu\right) \times \nu=$ $-\left(\boldsymbol{z}_{h} \times \nu\right) \tau$, where $\tau$ is the tangential vector.

Since $\nu$ is constant on each $\Gamma \in \Gamma_{h}$ with $|\nu|=1$,

$$
\mid \int_{\Gamma}\left[\boldsymbol{z}_{h}\right] d S\left(\left.x\right|^{2}=\left|\left(\int_{\Gamma}\left[\boldsymbol{z}_{h} \times \nu\right] d S(x)\right) \times \nu\right|^{2} \leq\left|\int_{\Gamma}\left[\boldsymbol{z}_{h} \times \nu\right] d S(x)\right|^{2}, \quad \forall \Gamma \in \Gamma_{h}^{I} .\right.
$$

As a result, applying (A.9) of Lemma A.5 to A.12) yields the error estimate

$$
\left\|\boldsymbol{z}_{h}-\Pi_{h}^{R} \boldsymbol{z}_{h}\right\|_{\boldsymbol{L}^{2}(\Omega)}^{2} \leq C\left(\sum_{E \in E_{h}} h^{2}\left\|D \boldsymbol{z}_{h}\right\|_{\boldsymbol{L}^{2}(\Omega)}^{2}+h^{\frac{4-N}{2}}\left\|\operatorname{div} \boldsymbol{z}_{h}\right\|_{L^{2}(\Omega)}^{2}\right) .
$$


To continue, fix an arbitrary $\xi \in \mathbb{R}^{N}$. By the triangle inequality, we write

$$
\begin{aligned}
& \left\|\boldsymbol{z}_{h}(\cdot)-\boldsymbol{z}_{h}(\cdot-\xi)\right\|_{L^{2}\left(\Omega_{\xi}\right)}^{2} \\
& \leq C\left(\left\|z_{h}(\cdot)-\Pi_{h}^{R} \boldsymbol{z}_{h}(\cdot)\right\|_{L^{2}\left(\Omega_{\xi}\right)}^{2}\right. \\
& +\left\|\Pi_{h}^{R} \boldsymbol{z}_{h}(\cdot)-\Pi_{h}^{R} \boldsymbol{z}_{h}(\cdot-\xi)\right\|_{L^{2}\left(\Omega_{\xi}\right)}^{2} \\
& \left.+\left\|\Pi_{h}^{R} \boldsymbol{z}_{h}(\cdot-\xi)-\boldsymbol{z}_{h}(\cdot-\xi)\right\|_{L^{2}\left(\Omega_{\xi}\right)}^{2}\right),
\end{aligned}
$$

which transfers the translation onto the projected function $\Pi_{h}^{R} \boldsymbol{z}$.

Since $\Pi_{h}^{R} \boldsymbol{z}_{h}$ is a function in the Crouzeix-Raviart element space, Stummel's work 27. Theorem 2.1] can be applied, yielding

$$
\begin{aligned}
& \left\|\Pi_{h}^{R} \boldsymbol{z}_{h}(\cdot)-\Pi_{h}^{R} \boldsymbol{z}_{h}(\cdot-\xi)\right\|_{\boldsymbol{L}^{2}\left(\Omega_{\xi}\right)}^{2} \\
& \quad \leq C\left(h^{2}+|\xi|^{2}\right) \sum_{E \in E_{h}}\left\|D \Pi_{h}^{R} \boldsymbol{z}_{h}\right\|_{\boldsymbol{L}^{2}(E)}^{2} \\
& \quad \leq C\left(h^{2}+|\xi|^{2}\right) \sum_{E \in E_{h}}\left\|D \boldsymbol{z}_{h}\right\|_{\boldsymbol{L}^{2}(E)}^{2},
\end{aligned}
$$

where the constant $C$ only depends on $\Omega$ and the shape regularity of $E_{h}$.

Utilizing A.13) and A.15 in a.14 gives

$$
\begin{aligned}
& \left\|\boldsymbol{z}_{h}(\cdot)-\boldsymbol{z}_{h}(\cdot-\xi)\right\|_{\boldsymbol{L}^{2}\left(\Omega_{\xi}\right)}^{2} \\
& \quad \leq C\left(h^{2}+|\xi|^{2}\right) \sum_{E \in E_{h}}\left\|D \boldsymbol{z}_{h}\right\|_{\boldsymbol{L}^{2}(E)}^{2}+h^{\frac{4-N}{2}}\left\|\operatorname{div} \boldsymbol{z}_{h}\right\|_{L^{2}(\Omega)}^{2} .
\end{aligned}
$$

Since $\left|D \boldsymbol{z}_{h}\right|=\left|\operatorname{div} \boldsymbol{z}_{h}\right|$ on each $E \in E_{h}$, this immediately yields

$$
\left\|\boldsymbol{z}_{h}(\cdot)-\boldsymbol{z}_{h}(\cdot-\xi)\right\|_{\boldsymbol{L}^{2}\left(\Omega_{\xi}\right)} \leq C\left(h^{\frac{4-N}{2}}+h^{2}+|\xi|^{2}\right)^{\frac{1}{2}}\left\|\operatorname{div} \boldsymbol{z}_{h}\right\|_{L^{2}(\Omega)},
$$

Finally, let us argue that A.16) implies A.1. To this end, fix any $\xi \in \mathbb{R}^{N}$ and $h>0$. There exists a shape regular partition $G_{h}$ of $\Omega$ into triangles/tetrahedra such that $\bigcup_{E \in G_{h}} E=\bigcup_{E \in E_{h}} E$, each $E \in G_{h}$ has a nonempty intersection with at most one element $E \in E_{h}$, and such that

$$
\max _{E \in G_{h}} \operatorname{diam}(E)<\frac{|\xi|}{3} .
$$

Next, let $\boldsymbol{V}_{|\xi|}(\Omega)$ denote the first order div conforming Nédélec element space of the first kind formed on the mesh $G_{h}$, and let $\Pi_{|\xi|}^{V}: V_{h}(\Omega) \rightarrow V_{|\xi|}(\Omega)$ denote the usual projection into this space.

Now, for any $\boldsymbol{z}_{h} \in \boldsymbol{V}_{h}^{0, \perp}(\Omega)$, we calculate

$$
\begin{aligned}
& \left\|\boldsymbol{z}_{h}(\cdot)-\boldsymbol{z}_{h}(\cdot-\xi)\right\|_{\boldsymbol{L}^{2}\left(\Omega_{\xi}\right)}^{2} \\
& \leq\left\|\boldsymbol{z}_{h}(\cdot)-\Pi_{|\xi|}^{V} \boldsymbol{z}_{h}(\cdot)\right\|_{\boldsymbol{L}^{2}\left(\Omega_{\xi}\right)}^{2}+\left\|\Pi_{|\xi|}^{V} \boldsymbol{z}_{h}(\cdot)-\Pi_{|\xi|}^{V} \boldsymbol{z}_{h}(\cdot-\xi)\right\|_{\boldsymbol{L}^{2}\left(\Omega_{\xi}\right)}^{2} \\
& \quad+\left\|\Pi_{|\xi|}^{V} \boldsymbol{z}_{h}(\cdot-\xi)-\boldsymbol{z}_{h}(\cdot-\xi)\right\|_{\boldsymbol{L}^{2}\left(\Omega_{\xi}\right)}^{2}
\end{aligned}
$$


By A.16,

$$
\begin{aligned}
& \left\|\Pi_{|\xi|}^{V} \boldsymbol{z}_{h}(\cdot)-\Pi_{|\xi|}^{V} \boldsymbol{z}_{h}(\cdot-\xi)\right\|_{\boldsymbol{L}^{2}\left(\Omega_{\xi}\right)}^{2} \\
& \quad \leq C\left(|\xi|^{\frac{4-N}{2}}+|\xi|^{2}\right)^{\frac{1}{2}}\left\|\operatorname{div} \boldsymbol{z}_{h}\right\|_{L^{2}(\Omega)} .
\end{aligned}
$$

By Lemma 2.11,

$$
\begin{aligned}
& \left\|\boldsymbol{z}_{h}(\cdot)-\Pi_{|\xi|}^{V} \boldsymbol{z}_{h}(\cdot)\right\|_{\boldsymbol{L}^{2}\left(\Omega_{\xi}\right)}^{2} \\
& \quad \leq C|\xi|^{2} \sum_{E \in E_{h}}\left\|D \boldsymbol{z}_{h}\right\|_{\boldsymbol{L}^{2}(\Omega)}^{2}=C|\xi|^{2}\left\|\operatorname{div} \boldsymbol{z}_{h}\right\|_{L^{2}(\Omega)}^{2} .
\end{aligned}
$$

Inserting (A.18) and (A.19) into (A.17) completes the proof of A.1).

\section{REFERENCES}

[1] S. Agmon. Lectures on elliptic boundary value problems. Van Nostrand, Princeton, 1965. MR0178246 (31:2504)

[2] D. N. Arnold, R. S. Falk, and R. Winther. Finite element exterior calculus, homological techniques, and applications. Acta Numer., 15:1-155, 2006. MR:2269741 (2007j:58002)

[3] D. N. Arnold, R. S. Falk, and R. Winther. Multigrid in H(div) and H(curl). Numer. Math., 85(2):197-217, 2000. MR1754719 (2001d:65161)

[4] S. C. Brenner and R. Scott. The mathematical theory of finite element methods, volume 15 of Texts in Applied Mathematics. Springer-Verlag, New York, 2002. MR1894376 (2003a:65103)

[5] S. C. Brenner, J. Cui, and L.-Y. Sung. A nonconforming finite element method for a two-dimensional curl-curl and grad-div problem. Numer. Math., 109(4):509-533, 2008. MR2407321 (2009c:65294)

[6] S. C. Brenner. Poincaré-Friedrichs inequalities for piecewise $H^{1}$ functions. SIAM J. Numer. Anal., 41(1):306-324 (electronic), 2003. MR1974504(2004d:65140)

[7] F. Brezzi and M. Fortin. Mixed and hybrid finite element methods, volume 15 of Springer Series in Computational Mathematics. Springer-Verlag, New York, 1991. MR1115205 (92d:65187)

[8] G.-Q. Chen, D. Hoff, and K. Trivisa. Global solutions of the compressible Navier-Stokes equations with large discontinuous initial data. Comm. Partial Differential Equations, 25(1112):2233-2257, 2000. MR.1789926 (2001h:35141)

[9] B. Cockburn. Discontinuous Galerkin methods for convection-dominated problems. In Highorder methods for computational physics, volume 9 of Lect. Notes Comput. Sci. Eng., pages 69-224. Springer, Berlin, 1999. MR1712278 (2000f:76095)

[10] B. Cockburn and C.-W. Shu. Runge-Kutta discontinuous Galerkin methods for convectiondominated problems. J. Sci. Comput., 16(3):173-261, 2001. MR1873283 (2002i:65099)

[11] S. Christiansen. Stability of Hodge decompositions in finite element spaces of differential forms in arbitrary dimension. Numer. Math. 107(1):87-106, 2007. MR2317829|(2008c:65318)

[12] K. Deimling. Nonlinear functional analysis. Springer-Verlag, Berlin, 1985. MR787404 (86j:47001)

[13] R. J. DiPerna and P.-L. Lions. Ordinary differential equations, transport theory and Sobolev spaces. Invent. Math., 98(3):511-547, 1989. MR1022305(90j:34004)

[14] E. Feireisl. Dynamics of viscous compressible fluids, volume 26 of Oxford Lecture Series in Mathematics and its Applications. Oxford University Press, Oxford, 2004. MR2040667 (2005i:76092)

[15] T. Gallouët, R. Herbin, and J.-C. Latché. A convergent finite element-finite volume scheme for the compressible Stokes problem. Part I: The isothermal case. Math. Comp, Online, 2009. MR2501053 (2010c:65213)

[16] T. Gallouët, L. Gestaldo, R. Herbin, and J.-C. Latché. An unconditionally stable pressure correction scheme for the compressible barotropic Navier-Stokes equations. M2AN Math. Model. Numer. Anal., 42(2):303-331, 2008. MR2405150(2009b:76125) 
[17] V. Girault and P.-A. Raviart. Finite element methods for Navier-Stokes equations, volume 5 of Springer Series in Computational Mathematics. Springer-Verlag, Berlin, 1986. Theory and algorithms. MR851383(88b:65129)

[18] D. Hoff. Global existence for 1D, compressible, isentropic Navier-Stokes equations with large initial data. Trans. Amer. Math. Soc., 303(1):169-181, 1987. MR896014 (88k:35162)

[19] D. Hoff. Discontinuous solutions of the Navier-Stokes equations for compressible flow. Arch. Rational Mech. Anal., 114(1):15-46, 1991. MR1088275 (92e:76052)

[20] P. Lasaint and P.-A. Raviart. On a finite element method for solving the neutron transport equation. In Mathematical aspects of finite elements in partial differential equations (Proc. Sympos., Math. Res. Center, Univ. Wisconsin, Madison, Wis., 1974), pages 89-123. Publication No. 33. Math. Res. Center, Univ. of Wisconsin-Madison, Academic Press, New York, 1974. MR0658142 (58:31918)

[21] P.-L. Lions. Mathematical topics in fluid mechanics. Vol. 1: Incompressible models. Oxford University Press, New York, 1996. MR1422251 (98b:76001)

[22] P.-L. Lions. Mathematical topics in fluid mechanics. Vol. 2: Compressible models. Oxford University Press, New York, 1998. MR 1637634 (99m:76001)

[23] C. Liu and N. J. Walkington. Convergence of numerical approximations of the incompressible Navier-Stokes equations with variable density and viscosity. SIAM J. Numer. Anal., 45(3):1287-1304 (electronic), 2007. MR2318813 (2008f:65177)

[24] J.-C. Nédélec. Mixed finite elements in $\mathbf{R}^{3}$. Numer. Math., 35(3):315-341, 1980. MR592160 (81k:65125)

[25] J.-C. Nédélec. A new family of mixed finite elements in $\mathbf{R}^{3}$. Numer. Math., 50(1):57-81, 1986. MR864305 (88e:65145)

[26] W. Reed and T. Hill. Triangular mesh methods for the neutron transport equation. Tech. Report LA-UR-73-479, Los Alamos Scientific Laboratory, 1973.

[27] F. Stummel. Basic compactness properties of nonconforming and hybrid finite element spaces. RAIRO Anal. Numér., 14(1):81-115, 1980. MR566091 (81h:65058)

[28] R. S. Varga. Matrix iterative analysis, volume 27 of Springer Series in Computational Mathematics. Springer-Verlag, New York, 2000. MR.1753713 (2001g:65002)

[29] N. J. Walkington. Convergence of the discontinuous Galerkin method for discontinuous solutions. SIAM J. Numer. Anal., 42(5):1801-1817 (electronic), 2005. MR2139223(2006b:65147)

[30] R. Zarnowski and D. Hoff. A finite-difference scheme for the Navier-Stokes equations of one-dimensional, isentropic, compressible flow. SIAM J. Numer. Anal., 28(1):78-112, 1991. MR:1083325 (92d:65176)

[31] J. Zhao and D. Hoff. A convergent finite-difference scheme for the Navier-Stokes equations of one-dimensional, nonisentropic, compressible flow. SIAM J. Numer. Anal., 31(5):1289-1311, 1994. MR1293516 (95j:65105)

[32] J. J. Zhao and D. Hoff. Convergence and error bound analysis of a finite-difference scheme for the one-dimensional Navier-Stokes equations. In Nonlinear evolutionary partial differential equations (Beijing, 1993), volume 3 of AMS/IP Stud. Adv. Math., pages 625-631. Amer. Math. Soc., Providence, RI, 1997. MR1468542

Centre of Mathematics for Applications, University of Oslo, P.O. Box 1053, BlinDern, N-0316 Oslo, Norway and Center for Biomedical Computing, Simula Research Laboratory, P.O. Box 134, N-1325 Lysaker, Norway

E-mail address: kennethk@math.uio.no

URL: http://folk.uio.no/kennethk

Centre of Mathematics for Applications, University of Oslo, P.O. Box 1053, BlinDERN, N-0316 OSLO, NoRWAY

E-mail address: t.k.karper@cma.uio.no

$U R L:$ http://folk.uio.no/trygvekk/ 